\title{
SUBELLIPTIC OPERATORS ON LIE GROUPS: VARIABLE COEFFICIENTS
}

\author{
Ola Bratteli \\ and \\ Derek W. Robinson
}




\section{Contents}

1 Introduction 1

2 Constant coefficients $\quad 10$

3 A priori estimates 14

4 Resolvent estimates for constant coefficients 25

5 The parametrix method 35

6 Semigroups on $C_{0}$ and $L_{\infty} \quad 43$

$\begin{array}{lll}7 & \text { Semigroups on } L_{1} & 49\end{array}$

8 Semigroups on $L_{p}, p \in\langle 1, \infty\rangle \quad \mathbf{5 5}$

$\begin{array}{llr}9 & \text { Holomorphy } & 59\end{array}$

$\begin{array}{lc}10 \text { Semigroup kernels } & 66\end{array}$

11 Generator Theorems $\quad 80$

12 Lower bounds 103

13 Conclusion $\quad 110$

$\begin{array}{ll}\text { A Appendix } & 112\end{array}$

$\begin{array}{lr}\text { References } & 116\end{array}$ 
Abstract

Let $G$ be a Lie group with Lie algebra $\mathfrak{g}$ and $a_{i}, \ldots, a_{d^{\prime}}$ an algebraic basis of $\mathfrak{g}$. Further, if $A_{i}=d L\left(a_{i}\right)$ are the corresponding generators of left translations by $G$ on one of the usual function spaces over $G$, let

$$
H=\sum_{\alpha ;|\alpha| \leq 2} c_{\alpha} A^{\alpha}
$$

be a second-order differential operator with real bounded coefficients $c_{\alpha}$. The operator is defined to be subelliptic if

$$
\inf \left\{-\sum_{\alpha ;|\alpha|=2} c_{\alpha}(g) \xi^{\alpha} ; g \in G, \xi \in \mathrm{R}^{d^{\prime}},|\xi|=1\right\}>0 .
$$

We prove that if the principal coefficients $\left\{c_{\alpha} ;|\alpha|=2\right\}$ of the subelliptic operätor are once left differentiable in the directions $a_{1}, \ldots, a_{d^{\prime}}$ with bounded derivatives then the operator has a family of semigroup generator extensions on the $L_{p}$-spaces with respect to left Haar measure $d g$, or right Haar measure $d \hat{g}$, and the corresponding semigroups $S$ are given by a positive integral kernel,

$$
\left(S_{t} \varphi\right)(g)=\int_{G} d \hat{h} K_{t}(g ; h) \varphi(h) .
$$

The semigroups are holomorphic and the kernel satisfies Gaussian upper bounds. If in addition the coefficients with $|\alpha|=2$ are three times differentiable and those with $|\alpha|=1$ are once differentiable then the kernel also satisfies Gaussian lower bounds.

Some original features of this article are the use of the following: a priori inequalities on $L_{\infty}$ in Section 3, fractional operator expansions for resolvent estimates in Section 4, a parametrix method based on reduction to constant coefficient operators on the Lie group rather than the usual Euclidean space in Section 5, approximation theory of semigroups in Section 11 and 'time dependent' perturbation theory to treat the lower order terms of $H$ in Sections 11 and 12.

AMS Subject Classification: 43A65, 22E45, 35H05, 22E25, 35B45. 


\section{Preface}

The major part of this work was carried out in 1989-1990 whilst the first author was on sabbatical leave at the Australian National University. It was completed during a subsequent visit in December 1991-January 1992. The article is a natural extension of the analysis of strongly elliptic operators with variable coefficients described in the book 'Elliptic Operators and Lie Groups' by the second author. A number of the techniques developed in the article were already incorporated in Chapter $\mathrm{V}$ of this book and the order of publication does not reflect the order of the work.

The early part of the work, and in particular the material of Sections 4-7, was helped by numerous discussions with Trond Digernes. We are also grateful to Oscar Lanford for discussions on the approximation methods of Section 11. 


\section{Introduction}

Our purpose is to develop the basic theory of second-order subelliptic differential operators with real coefficients acting on functions over a Lie group $G$. Under relatively mild smoothness hypotheses on the coefficients we demonstrate that these operators generate continuous semigroups on the usual $L_{p}$-spaces over $G$ and that these semigroups are determined by a smooth integral kernel. The kernel satisfies Gaussian upper and lower bounds. These results extend earlier work, begun with Arendt and Batty, on strongly elliptic operators [ABR] [BrR1] [Rob1]. Related results have also been given by Ouhabaz [Ouh], Norris and Stroock [NoS], Saloff-Coste and Stroock $[\mathrm{SaS}]$ and Stroock [Str].

Let $G$ denote a Lie group and $G_{0}$ the connected component of the identity $e$. Further let $d g$ denote left-invariant Haar measure and $g \mapsto \Delta(g)$ the modular function, i.e., the function such that $\int_{G} d h \varphi(h g)=\Delta(g)^{-1} \int_{G} d h \varphi(h)$ for all integrable functions $\varphi$. Then $d \hat{g}=\Delta(g)^{-1} d g$ is right-invariant Haar measure, and $\int_{G} d h \varphi(h)=$ $\int_{G} d \hat{h} \varphi\left(h^{-1}\right)$. Next let $L_{p}=L_{p}(G ; d g)$ be the usual $L_{p}$-spaces over $G$ formed with respect to the left-invariant measure and $L_{\hat{p}}=L_{p}(G ; d \hat{g})$ the corresponding spaces formed with the right-invariant measure. We use $\|\cdot\|_{p}$, and $\|\cdot\|_{\hat{p}}$, to denote the corresponding norms, e.g., if $p \in[1, \infty\rangle$ then

$$
\|\varphi\|_{p}=\left(\int_{G} d g|\varphi(p)|^{p}\right)^{1 / p}
$$

for $\varphi \in L_{p}$ and

$$
\|\hat{\varphi}\|_{\hat{p}}=\left(\int_{G} d \hat{g}|\hat{\varphi}(p)|^{p}\right)^{1 / p}
$$

for $\hat{\varphi} \in L_{\hat{p}}$. The spaces $L_{\infty}$ and $L_{\dot{\infty}}$ are identical and

$$
\|\varphi\|_{\infty}=\operatorname{ess} \sup \{|\varphi(g)| ; g \in G\} \text {. }
$$

Finally $C_{0}=C_{0}(G)$ is the Banach space of continuous functions over $G$ which vanish at infinity equipped with the supremum norm.

The group $G$ acts by left translations $L$ on each of the foregoing spaces. Explicitly

$$
(L(g) \varphi)(h)=\varphi\left(g^{-1} h\right)
$$


for all $g, h-\in G$. This action is strongly continuous on $L_{p}$, or $L_{\hat{p}}$, for $p \in[1, \infty\rangle$, or on $C_{0}$, and weakly* continuous on $L_{\infty}$. In order to handle the subsequent analysis of differential operators in a uniform manner it will be understood that all references to topological properties, density, continuity etc. will be with respect to the weak* topology on $L_{\infty}$ and the strong topology in all other cases. For example, if $a$ is an element of the Lie algebra $\mathfrak{g}$ of $G$ and $A=d L(a)=\lim _{t \rightarrow 0}\left(I-L\left(e^{-t a}\right)\right) / t$ is the infinitesimal generator of the one-parameter group $t \in \mathbf{R} \mapsto L\left(e^{-t a}\right)$, then $A$ is weak* densely defined and weak ${ }^{*}$-weak ${ }^{*}$-closed on $L_{\infty}$ but norm densely defined and norm closed on the other spaces. We will refer to it in brief as a densely defined, closed operator.

Let $a_{1}, \ldots, a_{d}$ be a linear basis for $\mathfrak{g}$ and set $A_{i}=d L\left(a_{i}\right)$. Then define the dense subspaces

$$
L_{p ; n}=\bigcap_{0 \leq m \leq n} \bigcap_{1 \leq i_{1}, \ldots, i_{m} \leq d} D\left(A_{i_{1}} \ldots A_{i_{m}}\right)
$$

of $L_{p}$ and introduce the norms $\|\cdot\|_{p ; n}$

$$
\|\varphi\|_{p ; n}=\sup _{0 \leq m \leq n} N_{p ; m}(\varphi)
$$

for $\varphi \in L_{p ; n}$ where the seminorms $N_{p ; m}$ are defined recursively by $N_{p ; 0}(\varphi)=\|\varphi\|_{p}$ and

$$
N_{p ; m+1}(\varphi)=\sup _{1 \leq i \leq d} N_{p ; m}\left(A_{i} \varphi\right)
$$

The subspaces $L_{\hat{p} ; n}$ and $C_{0 ; n}$ are defined analogously. Each of these spaces is a Banach space with respect to the corresponding norm. The space $L_{p ; n}$ corresponds to the $n$ times $L_{p}$-differentiable functions. Now as $L$ is weakly* continuous on $L_{\infty}$ a function $\varphi \in L_{\infty}$ is once $L_{\infty}$-differentiable if, and only if,

$$
\sup _{0<t \leq 1}\left\|\left(I-L\left(e^{-t a}\right)\right) \varphi\right\|_{\infty} / t<\infty
$$

for all $a \in \mathfrak{g}$, [BrR2]. Equivalently, $\varphi \in L_{\infty ; 1}$ if, and only if,

$$
\sup \left\{\left|\varphi(g)-\varphi\left(h^{-1} g\right)\right| /|h| ; g \in G,|h| \leq 1\right\}<\infty
$$

where the modulus $|\cdot|$ is defined in terms of a right-invariant distance $d$ on $G_{0}$ by $|g|=$ $d(e ; g)$. Thus $L_{\infty ; 1}$ is exactly the space of bounded uniformly Lipschitz continuous functions. In particular $L_{\infty ; 1} \subseteq C_{b}$, the space of bounded continuous functions over $G$, and it acts by multiplication on each of the above spaces $L_{p}, p \in[1, \infty]$, and $C_{0}$. 
Next let $a_{1}, \ldots, a_{d^{\prime}}$ denote an algebraic basis of $\mathfrak{g}$, i.e., the $a_{1}, \ldots, a_{d^{\prime}}$ are linearly independent and together with their repeated commutators $\left(\operatorname{ad} a_{i_{1}}\right) \ldots\left(\operatorname{ad} a_{i_{m}}\right)\left(a_{i_{m+1}}\right)$, $1 \leq i_{1}, \ldots, i_{m+1} \leq d^{\prime}, m=1,2, \ldots, n$, span $\mathfrak{g}$ linearly. The smallest $n$ for which this is true is called the rank of the algebraic basis. In particular a linear basis has rank zero. One can then introduce spaces

$$
L_{p ; n}^{\prime}=\bigcap_{0 \leq m \leq n} \bigcap_{1 \leq i_{1}, \ldots, i_{m} \leq d^{\prime}} D\left(A_{i} \ldots A_{i_{m}}\right)
$$

seminorms $N_{p ; n}^{\prime}$, and norms $\|\cdot\|_{p ; n}^{\prime}$, in terms of the sub-basis by repetition of the previous definitions. Similarly one defines $L_{\hat{p} ; n}^{\prime},\|\cdot\|_{\hat{p} ; n}^{\prime}$, etc. The space $L_{p ; 1}^{\prime}$ consists of the functions which are differentiable in the $L_{p^{p}}$-sense in the directions $a_{1}, \ldots, a_{d^{\prime}}$ but if $n$ is larger than the rank of the algebraic basis then elements of $L_{p ; n}^{\prime}$ will be differentiable in all directions. The subspaces $C_{0 ; n}^{\prime}$ of $C_{0}$ are defined analogously, and in fact one may introduce the spaces $\mathcal{X}_{n}^{\prime \prime}$ for any Banach space $\mathcal{X}$ upon which $G$ acts continuously.

Now, following [Car] [Bon], we introduce a canonical distance corresponding to the algebraic basis. Let $\gamma:[0,1] \mapsto G$ be a continuous piecewise differentiable path from $g$ to $h$ such that the tangents to the smooth subsegments are in the subspace spanned by $a_{1}, \ldots, a_{d^{\prime}}$. Hence there exist scalar functions $\gamma_{i}$ on $[0,1]$ such that

$$
\frac{d \psi(\gamma(t))}{d t}=\sum_{i=1}^{d^{\prime}} \gamma_{i}(t)\left(A_{i} \psi\right)(\gamma(t))
$$

for all $\psi \in C_{c}^{\infty}(G)$. Then we define the right-invariant distance $d^{\prime}$ by

$$
d^{\prime}(g ; h)=\inf _{\gamma} \int_{0}^{1} d t\left(\sum_{i=1}^{d^{\prime}} \gamma_{i}(t)^{2}\right)^{1 / 2}
$$

and the associated modulus $\mid \cdot l^{\prime}$ by

$$
|g|^{\prime}=d^{\prime}(e ; g)
$$

if $g \in G_{0}$. We define $|g|^{\prime}=\infty$ if $g \in G \backslash G_{0}$. By right-invariance, $d^{\prime}(g ; h)=\left|h g^{-1}\right|^{\prime}$. Now if $|\cdot|$ is the corresponding modulus for a full basis containing the algebraic basis it follows that $|g| \leq|g|^{\prime}$ for all $g \in G_{0}$. But conversely there is a constant $k>0$ and a constant $\delta \in\langle 0,1]$ such that $|g|^{\prime} \leq k|g|^{\delta}$ for all $g$ in $G_{0}$ with $|g| \leq 1$. The value of $\delta$ is determined by the rank $n$ of the algebraic basis through the relation $\delta=1 /(n+1)$ but this identification will not be used in the sequel. In addition there is a $D^{\prime} \geq d$ such that the Haar measure $\left|B_{r}^{\prime}\right|$ of the ball $B_{r}^{\prime}=\left\{g \in G ;|g|^{\prime} \leq r\right\}$ satisfies bounds 
$k^{-1} r^{D^{\prime}} \leq\left|B_{r}^{\prime}\right| \leq k r^{D^{\prime}}$ for some $k>0$ and all $r \in\langle 0,1]$. Thus $D^{\prime}$ corresponds to the local dimension of $G$ with respect to the algebraic basis. The local dimension $D^{\prime}$ can be defined algebraically as follows: If $\mathfrak{g}_{0}=\{0\}, \mathfrak{g}_{1}$ is the linear span of the algebraic basis $a_{1}, \ldots, a_{d^{\prime}}$ and $\mathfrak{g}_{j+1}$ the linear span of the algebraic basis together with the corresponding multiple commutators of order less than or equal to $j$, then

$$
D^{\prime}=\sum_{j=1}^{n+1} j \operatorname{dim}\left(\mathfrak{g}_{j} / \mathfrak{g}_{j-1}\right)
$$

where $n$ is the rank of the algebraic basis. If $\varphi \in L_{\infty ; 1}^{\prime}$ one then has

$$
\varphi(h)-\varphi(g)=\int_{0}^{1} d t \sum_{i=1}^{d^{\prime}} \gamma_{i}(t)\left(A_{i} \varphi\right)(\gamma(t))
$$

and this gives bounds

$$
|\varphi(h)-\varphi(g)| \leq k\left|h g^{-1}\right|^{\prime} N_{\infty ; 1}^{\prime}(\varphi) \leq k^{\prime}\left|h g^{-1}\right|^{\delta} N_{\infty ; 1}(\varphi)
$$

where the second inequality presumes $\left|h g^{-1}\right| \leq 1$. Thus elements of $L_{\infty ; 1}^{\prime}$ are Lipschitz continuous with exponent one or $\delta$ according to the choice of modulus. In particular $L_{\infty ; 1}^{\prime} \subseteq C_{b}$ and $L_{\infty ; 1}^{\prime}$ acts by multiplication on each of the function spaces $L_{p}, L_{\hat{p}}, p \in$ $[1, \infty]$, and $C_{0}$.

Now fix a sub-basis $a_{1}, \ldots, a_{d^{\prime}}$ of $\mathfrak{g}$, i.e., a linearly independent subset of $\mathfrak{g}$. Then a second-order left differential operator $H$ on $L_{p}$, or $L_{\hat{p}}$, with domain of definition $L_{p ; 2}$, or $L_{\hat{p} ; 2}$, is defined by

$$
H=-\sum_{i, j=1}^{d^{\prime}} c_{i j} A_{i} A_{j}+\sum_{i=1}^{d^{\prime}} c_{i} A_{i}+c_{0}
$$

where $c_{0}, c_{i}, c_{i j} \in L_{\infty}$ and the matrix $\left(c_{i j}\right)$ is symmetric. It is, however, convenient to use the notation

$$
H=\sum_{\alpha ;|\alpha| \leq 2} c_{\alpha} A^{\alpha}
$$

where $\alpha \in\left\{1, \ldots, d^{\prime}\right\}^{n}$ for some $n=0,1,2,|\alpha|=n$, and the coefficients $c_{\alpha}$ together with the products $A^{\alpha}$ of left derivatives are identified in the obvious way. Since we assume the matrix of second-order coefficients is symmetric the $c_{\alpha}$ are uniquely determined by $H$. 
The operator $H$ is said to be subelliptic if $a_{1}, \ldots, a_{d^{\prime}}$ is an algebraic basis, the coefficients $c_{\alpha}$ are real and

$$
\lambda_{c}=\operatorname{essinf}\left\{-\sum_{\alpha ;|\alpha|=2} c_{\alpha}(g) \xi^{\alpha} ; g \in G, \xi \in \mathbf{R}^{d^{\prime}},|\xi|=1\right\}>0 .
$$

Thus the matrix $\left(c_{i j}\right)$ is uniformly strictly positive-definite. The parameter $\lambda_{c}$ is called the ellipticity constant of $H$ and we also define $\Lambda_{c}$ by

$$
\Lambda_{c}=\operatorname{ess} \sup \left\{-\sum_{\alpha ;|\alpha|=2} c_{\alpha}(g) \xi^{\alpha} ; g \in G, \xi \in \mathbf{R}^{d^{\prime}},|\xi|=1\right\} .
$$

If, in addition, $a_{1}, \ldots, a_{d^{\prime}}$ is a vector space basis of $\mathfrak{g}$ then $H$ is said to be strongly elliptic. The theory of strongly elliptic operators of this type has been developed in Chapter V of [Rob1] and our aim is to extend the principal conclusions to the subelliptic case. This requires a variety of new techniques.

If $c_{\alpha} \in L_{\infty ; 1}^{\prime}$ for $|\alpha|=1,2$ then the subelliptic operator (1.17) can be expressed in the symmetric form

$$
H=-\sum_{i, j=1}^{d^{\prime}} A_{i} c_{i j} A_{j}+\sum_{i=1}^{d^{\prime}}\left(c_{i} A_{i}+A_{i} c_{i}\right) / 2+c_{0}
$$

or, by redefinition of $c_{0}$, in the partially symmetric forms

$$
\begin{aligned}
& H=-\sum_{i, j=1}^{d^{\prime}} A_{i} c_{i j} A_{i}+\sum_{i=1}^{d^{\prime}} c_{i} A_{i}+c_{0}, \\
& H=-\sum_{i, j=1}^{d^{\prime}} A_{i} c_{i j} A_{j}+\sum_{i=1}^{d^{\prime}} A_{i} c_{i}+c_{0} .
\end{aligned}
$$

The subellipticity condition corresponds to uniform positive-definiteness of the matrix of functions $\left(c_{i j}\right)$. In particular if $\lambda_{c}(g)$, and $\Lambda_{c}(g)$, are the smallest, and largest, eigenvalues of $\left(c_{i j}(g)\right)$ then $\lambda_{c} \leq \lambda_{c}(g) \leq \Lambda_{c}(g) \leq \Lambda_{c}$ and

$$
\lambda_{c}=\underset{g \in G}{\operatorname{essinf}} \lambda_{c}(g), \quad \Lambda_{c}=\underset{g \in G}{\operatorname{esssup}} \Lambda_{c}(g) .
$$

The form (1.17) is particularly suited for discussion of $H$ on $L_{1}, L_{\hat{1}}, L_{\infty}$, or $C_{0}$, but the symmetric form (1.20) is adapted to the analysis of $H$ on $L_{p}$, or $L_{\hat{p}}$, with $p \in\langle 1, \infty\rangle$. In this latter context duality considerations are useful and the adjoint $H^{*}$ of $H$ on $L_{p}, p \in[1, \infty\rangle$, extends the formal adjoint

$$
H^{\dagger}=-\sum_{i, j=1}^{d^{\prime}} A_{i} c_{i j} A_{j}-\sum_{i=1}^{d^{\prime}}\left(c_{i} A_{i}+A_{i} c_{i}\right) / 2+c_{0}
$$


on $L_{q}$ with-domain of definition $L_{q ; 2}^{\prime}$ where $q$ is conjugate to $p$. Thus $H$ has a densely defined adjoint and this implies that it is closable. Alternatively if $H$ is considered as an operator on $L_{\hat{p}}, p \in[1, \infty\rangle$, then since $A_{i}^{*}=-A_{i}+\beta_{i} I$ with $\beta_{i}=\left(A_{i} \Delta\right)(e)$ it again follows that $H$ has a densely defined adjoint and is closable. Finally if $H$ acts on $L_{\infty}$ then the adjoint $\left(H^{\dagger}\right)^{*}$ of the formal adjoint $H^{\dagger}$ on $L_{1}$ is a weak ${ }^{*}$-weak ${ }^{*}$-closed extension of $H$, and hence $H$ is closable.

The space $C_{0}$ is rather special in that the operator (1.17) is not well defined on $C_{0 ; 2}^{\prime}$ unless the $c_{\alpha}$ are continuous bounded functions. But in that case $H$ is a bounded perturbation of the dissipative operator

$$
\sum_{\alpha_{j}|\alpha|=1,2} c_{\alpha} A^{\alpha}
$$

and hence it is closable.

Our primary interest is to decide whether $H$ has closed extensions which generate continuous semigroups on $C_{0}$, and $L_{\infty}$, but this automatically entails examination of the generation properties of $H$ on the other $L_{p}$-spaces. We first define an interpolating semigroup on the $L_{p}$-spaces to be a family of continuous semigroups $S=\left\{S^{(p)} ; p \in\right.$ $[1, \infty]\}$ where $S^{(p)}$ acts on $L_{p}$ and

$$
S_{t}^{(p)} \varphi=S_{t}^{(q)} \varphi
$$

for all $t>0$, all $\varphi \in L_{p} \cap L_{q}$, and all pairs $p, q \in[1, \infty]$. Then we use the common notation $S$ for the action of each semigroup. Secondly, we define a consistent interpolating semigroup to be a pair of interpolating semigroups $S$, and $\hat{S}$, on the $L_{p^{-}}$, and $L_{\hat{p}^{-}}$, spaces, respectively, satisfying

$$
S_{t} \varphi=\hat{S}_{t} \varphi
$$

for all $t>0$ all $\varphi \in L_{p} \cap L_{\hat{p}}$, and all $p \in[1, \infty]$. Again we use the common notation $S$ for the action. In addition $S$ is said to be positive if it maps positive functions into positive functions. Finally $S$ is defined to be holomorphic if it is holomorphic on each of the spaces with a common domain of holomorphy, i.e., if there exists a $\delta>0$ such that $t>0 \mapsto S_{t}$ extends to an analytic function in $\Delta_{\delta}=\{z \in \mathrm{C} ;|\arg z|<\delta\}$, with the standard properties, on each of the $L_{p^{-}}$, or $L_{\hat{p}^{-}}$, spaces.

Now, define $\mathcal{L}_{\hat{p}}$ as the closure of $L_{\infty} \otimes L_{\hat{p}}$ with respect to the norm

$$
\|\mid \varphi\|_{\hat{p}}=\operatorname{ess} \sup _{g \in G}\left(\int_{G} d \hat{h}|\varphi(g ; h)|^{p}\right)^{1 / p}
$$


if $p=[1, \infty\rangle$, or with respect to

$$
\||\varphi|\|_{\infty}=\underset{g, h \in G}{\operatorname{ess} \sup }|\varphi(g ; h)|
$$

if $p=\infty$. The semigroup $S$ on $L_{\infty}$ is defined to have a kernel $K$ if for each $t>0$ there is a $K_{t} \in \mathcal{L}_{\hat{1}}$ such that

$$
\left(S_{t} \varphi\right)(g)=\int_{G} d \hat{h} K_{t}(g ; h) \varphi(h)
$$

for each $\varphi \in L_{\infty}$. It follows that the norm of $S_{t}$ on $L_{\infty}$ is

$$
\left\|S_{t}\right\|_{\infty \rightarrow \infty}=\left\|\mid K_{t}\right\|_{\hat{1}} .
$$

In addition, if $K_{t} \in \mathcal{L}_{\hat{p}}$ then $S$ is bounded as an operator from $L_{\hat{q}}$ to $L_{\infty}$ and

$$
\left\|S_{t}\right\|_{\hat{q} \rightarrow \infty}=\left\|\mid K_{t}\right\|_{\hat{p}}
$$

where $p$ and $q$ are dual variables, i.e., $1 / p+1 / q=1$.

The properties of $S$ immediately place several restraints on the kernel, e.g., positivity of $S$ implies pointwise positivity of $K$ and the semigroup property gives

$$
K_{s+t}=K_{s} * K_{t}
$$

where the convolution product $*$ is defined by

$$
(\varphi * \psi)(g ; k)=\int_{G} d \hat{h} \varphi(g ; h) \psi(h ; k) .
$$

The group $G \times G$ acts by left translation on $\mathcal{L}_{\hat{p}}$ by

$$
(L(k ; l) \varphi)(g ; h)=\varphi\left(k^{-1} g ; l^{-1} h\right) .
$$

One can then define the subspace $\mathcal{L}_{\hat{p} ; 1}^{\prime}$, which corresponds to the product algebraic basis $\left(a_{1}, \ldots, a_{d^{\prime}}\right) \times\left(a_{1}, \ldots, a_{d^{\prime}}\right)$ of $\mathfrak{g} \times \mathfrak{g}$, as the common domain of $d L\left(a_{i}\right) \otimes I$, $I \otimes d L\left(a_{j}\right)$, for $i, j=1, \ldots, d^{\prime}$. At this point we can state a theorem which describes the main thrust of our results.

Theorem 1.1 Let $H$ be a subelliptic operator,

$$
H=\sum_{\alpha ;|\alpha| \leq 2} c_{\alpha} A^{\alpha}
$$

with coefficients $c_{\alpha} \in L_{\infty}$. 
1. If $c_{\alpha}^{-} \in L_{\infty ; 1}^{\prime}$ whenever $|\alpha|=2$ then $H$, on $L_{\infty}$, has a closed extension $\widetilde{H}$ which generates a continuous holomorphic semigroup $S$. The semigroup interpolates consistently between the $L_{p^{-}}$, and $L_{\hat{p}^{-}}$, spaces. In addition $S_{t} C_{0} \subseteq C_{0 ; 1}^{\prime}$ and the restriction of $S$ to $C_{0}$ is strongly continuous. The semigroup has a positive kernel $K_{t}$ and there exist $a, b>0$ and $\omega \geq 0$ such that

$$
K_{t}(g ; h) \leq a t^{-D^{\prime} / 2} e^{\omega t} e^{-b\left(\left|g h^{-1}\right|^{\prime}\right)^{2} t^{-1}}
$$

for all $g, h \in G$ and $t>0$.

2. If $c_{\alpha} \in L_{\infty ; 3}^{\prime}$ whenever $|\alpha|=2$ then $\widetilde{H}=\bar{H}$, the $L_{\infty}$-closure of $H$.

3. If $c_{\alpha} \in L_{\infty ; 3}^{\prime}$ whenever $|\alpha|=2$ and $c_{\alpha} \in L_{\infty ; 1}^{\prime}$ whenever $|\alpha|=1$ then there exist $a^{\prime}, b^{\prime}>0$, and $\omega^{\prime} \geq 0$, such that

$$
K_{t}(g ; h) \geq a^{\prime} t^{-D^{\prime} / 2} e^{-\omega^{\prime} t} e^{-b^{\prime}\left(\left|g h^{-1}\right|^{\prime}\right)^{2} t^{-1}}
$$

for all $g, h \in G_{0}$ and $t \geq 0$.

The theorem establishes a natural hierarchy of semigroup properties for the subelliptic operator governed by the smoothness of its coefficients. The semigroup properties are somewhat understated for the sake of brevity. For example, under the hypotheses of Statement 1 the derivatives in the subelliptic directions of the functions $S_{t} C_{0}, t>0$, are all Lipschitz continuous with exponent arbitrarily close to one. This refinement follows from Propositions 11.1 and 11.2. Although the theorem is similar to the results derived for strongly elliptic operators in [Rob1], Chapter 5, it requires stronger smoothness assumptions on the coefficients of $H$, especially for the Gaussian lower bounds on the kernels. This weakness can be traced to our inability to derive strong a priori estimates on $L_{\infty}$. It is important at several stages to establish that the left derivatives in the directions $a_{1}, \ldots, a_{d^{\prime}}$ are small perturbations of the subelliptic operator $H$ with bounds that have a weak dependence on the smoothness of the coefficients $c_{\alpha}$. This is easy to establish on $L_{2}$, or $L_{\hat{2}}$, but appears to be considerably more difficult on the other $L_{p}$-spaces. In Section 3 we derive an $L_{\infty}$-version of the perturbation estimates which only requires the principal coefficients of $H$ to be in $L_{\infty ; 1}^{\prime}$ but we have not been able to derive similar $L_{1}$-estimates. If, however, one has estimates of this type then the statements of the theorem can be considerably improved. For example, one could derive the Gaussian lower bounds, for $H$ in the partially asymmetric form (1.21), under the weaker hypotheses $c_{i j}, c_{i} \in L_{\infty ; 1}^{\prime}$ if the 
following conjecture were valid on $L_{\infty}$. The conjecture is formulated with a norm on the coefficients of $H$,

$$
\|c\|_{1}^{\prime}=\sum_{\alpha ;|\alpha|=2}\left\|c_{\alpha}\right\|_{\infty ; 1}^{\prime}+\sum_{\alpha ;|\alpha| \leq 1}\left\|c_{\alpha}\right\|_{\infty},
$$

which only involves derivatives of the leading coefficients.

$L_{\infty}$-conjecture If $H$ is a subelliptic operator of the form (1.17) and $c_{\alpha} \in L_{\infty ; 1}^{\prime}$ whenever $|\alpha|=2$ then there is an $a>0$, whose value depends on the coefficients of $H$ only through the parameters $\lambda_{c}$ and $\|c\|_{1}^{\prime}$, such that

$$
N_{\infty ; 1}^{\prime}(\varphi) \leq \varepsilon\|H \varphi\|_{\infty}+a \varepsilon^{-1}\|\varphi\|_{\infty}
$$

for all $\varphi \in L_{\infty ; 2}^{\prime}$ and all $\varepsilon \in\langle 0,1]$.

In Section 3 we derive a version of this conjecture in which the factor $\varepsilon^{-1}$ is replaced by $\varepsilon^{-1-\delta}$ for any $\delta>0$ but then the value of $a$ depends on $\delta$. This weak form of the conjecture is fundamental for the proof of Theorem 1.1 but the presence of the $\delta$-factor restricts our ability to derive Gaussian lower bounds under the hypotheses of Statement 1 of the theorem.

There are ample grounds for believing that the $L_{\infty}$-conjecture is valid. For example, if $H$ is strongly elliptic then it follows by a duality argument from a similar result on $L_{1}$ which is established in [Rob1] Chapter 5, Section 5. Alternatively, Proposition 3.1 gives the $L_{2^{-}}$, and $L_{\hat{2}^{-}}$, versions of the conjectures and Proposition 3.2 gives the slightly weakened form of the $L_{\infty}$-conjecture with the factor $\varepsilon^{-1-\delta}$. In addition, if the leading coefficients of $H$ are three times differentiable then the $L_{\infty}$-conjecture is established in Section 6 with the value of the constant $a$ depending on the norms $\left\|c_{\alpha}\right\|_{\infty ; 3}$ for $\alpha=2$.

The proof of the theorem is spread over the next eleven sections and in the course of the proofs we derive many more detailed results and estimates. In particular estimates on the derivatives of the kernel and continuity properties of $S$ as a function of the coefficients $c_{\alpha}$ play an important role. The key elements of the proof is contained in Sections 3,10, 11 and 12. In Section 2 we merely summarize some known results for operators with constant coefficients. Sections 4-9 describe the semigroup theory for operators with smooth, or at least relatively smooth, coefficients. The discussion is based on the parametrix method and is somewhat technical.

The reader who is ready to accept that operators with smooth coefficients generate interpolating semigroups can omit these sections. 


\section{Constant coefficients}

The starting point of our analysis of subelliptic operators with variable coefficients is the theory of analogous operators with constant coefficients. Theorem 1.1 has already been proved for this special case and much more is known [Jør] [San] [Rob1] [Var]. We begin by recalling some of the details which are of subsequent utility.

First, let $(\mathcal{X}, G, U)$ be a continuous representation of $G$ by bounded operators $U$ on the Banach space $\mathcal{X}$ and $A_{i}=d U\left(a_{i}\right)=\lim _{t \rightarrow 0}\left(I-U\left(e^{-t a_{i}}\right)\right) / t$ the corresponding generators: Then Jørgensen [Jør] has shown that each operator

$$
H_{U}=-\sum_{i=1}^{d^{\prime}} A_{i}^{2}+\sum_{i=1}^{d^{\prime}} c_{i} A_{i}
$$

with $c_{i} \in \mathrm{R}$ generates a continuous semigroup $S^{U}$ with a positive representation independent kernel $K$ such that

$$
S_{t}^{U} x=\int_{G} d h K_{t}(h) U(h) x
$$

for all $x \in \mathcal{X}$. But if one specializes to the representation of $G$ by left translations on the function spaces $L_{p}$, or $L_{\hat{p}}$, we now argue that this expression agrees with (1.30) for the semigroup $S$ whenever the coefficients of $H$ are constant. In this case $H$ automatically commutes with right translations. Therefore the associated semigroup is also invariant under right translations and this implies that the kernel $K$ satisfies

$$
K_{t}(g ; h)=K_{t}(g k ; h k)
$$

for all $g, h, k \in G$. Hence defining a function $K$ over $\mathrm{R}_{+} \times G$ by

$$
K_{t}(g)=K_{t}(g ; e)
$$

one has

$$
\begin{aligned}
\left(S_{t} \varphi\right)(g) & =\int_{G} d \hat{h} K_{t}(g ; h) \varphi(h) \\
& =\int_{G} d \hat{h} K_{t}\left(g h^{-1}\right) \varphi(h)=\int_{G} d h K_{t}(h) \varphi\left(h^{-1} g\right)
\end{aligned}
$$


Therefore $S$ is related to left translations $L$ by a formula analogous to $(2.2)$,

$$
S_{t}=\int_{G} d h K_{t}(h) L(h)=L\left(K_{t}\right) .
$$

Conversely, one can pass from (2.6) to (1.30) by setting

$$
K_{t}(g ; h)=K_{t}\left(g h^{-1}\right) .
$$

The kernel $K$ associated with $S^{U}$ is a positive $C^{\infty}$-function over $G$ which is representation independent [Jør] [Robl]. Moreover, since a continuous representation $U$ can have an exponential growth, i.e., $\|U(g)\| \sim e^{\rho|g|}$ for some $\rho \geq 0$, this result includes estimates

$$
\int_{G} d h K_{t}(h) e^{\rho|h|}<\infty
$$

for all $\rho \geq 0$. But these smoothness and boundedness results can be greatly improved [JeS] [Robl] [San] [Var], especially if the first-order terms in (2.1) are absent, i.e., if $c_{i}=0$.

Let $K^{0}$ denote the kernel for the special case that there are no first order terms, i.e., the kernel corresponding to the sublaplacian $-\sum_{i=1}^{d^{\prime}} A_{i}^{2}$. One has bounds

$$
\left|\left(A_{i_{1}} \ldots A_{i_{m}} K_{t}^{0}\right)(g)\right| \leq a_{m} t^{-D^{\prime} / 2} t^{-m / 2} e^{\omega t} e^{-b\left(|g|^{\prime}\right)^{2} / t}
$$

for all $t>0, g \in G$, and $1 \leq i_{1}, \ldots, i_{m} \leq d^{\prime}$, where $a_{m}, b>0$ and $\omega \geq 0$. Here $D^{\prime}$ is the local dimension of $G$ with respect to the algebraic basis $a_{1}, \ldots, a_{d^{\prime}}$ (see 1.14). These bounds are given explicitly in [JeS] for a stratified Lie algebra with $a_{1}, \ldots, a_{d^{\prime}}$ a vector space basis of the first component, and in [VSC], Chapter V, for $G$ unimodular. But the general result follows for small $t$ from Varopoulos' parabolic Harnack inequality [Var]. This inequality states that there is an $a_{m}>0$ and an $r>0$ such that for each $h \in G$

$$
\sup _{g \in B_{r \sqrt{t}}^{\prime}(h)}\left|\left(A_{i_{1}} \ldots A_{i_{m}} K_{t}^{0}\right)(g)\right| \leq a_{m} t^{-m / 2} \inf _{g \in B_{r \sqrt{t}}^{\prime}(h)} K_{2 t}^{0}(g)
$$

for all $t \in\langle 0,1]$ where

$$
B_{r}^{\prime}(h)=\left\{g ;\left|g h^{-1}\right|^{\prime} \leq r\right\} .
$$

(Varopoulos establishes this type of inequality for vector fields $A_{i}$ on a manifold and then the value of $a_{m}$ depends on the point $h$ in the manifold. But on a Lie group $a_{m}$ 
can be chosen independently of $h$ by translation invariance. A full discussion of these Harnack style inequalities is given in [VSC].) Therefore setting $g=h$ in (2.10) one has

$$
\left|\left(A_{i_{1}} \ldots A_{i_{m}} K_{t}^{0}\right)(g)\right| \leq a_{m} t^{-m / 2} K_{2 t}^{0}(g)
$$

for all $t \in\langle 0,1]$ and $g \in G$. But in addition one has estimates (see, for example, [Rob1] Chapter IV)

$$
K_{2 t}^{0}(g) \leq a t^{-D^{\prime} / 2} e^{\omega t} e^{-b\left(|g|^{\prime}\right)^{2} / t}
$$

for all $g \in G$ and $t>0$ with $a, b>0$ and $\omega \geq 0$, where $\omega$ can be chosen to be zero if $G$ is unimodular. Now (2.9), for $t \in\langle 0,1]$, follows by direct combination of $(2.11)$ and (2.12). If I however, $t \geq 1$ one can obtain the estimate from the small $t$ result by use of the convolution semigroup property of the kernel. This gives

$$
\left|\left(A_{i_{1}} \ldots A_{i_{m}} K_{t}^{0}\right)(g)\right| \leq \int_{G} d h\left|\left(A_{i_{1}} \ldots A_{i_{m}} K_{s}^{0}\right)(g h)\right| \cdot K_{t-s}^{0}\left(h^{-1}\right)
$$

for all $s \in\langle 0, t\rangle$. Then setting $s=1 / 2$ and using (2.9) and (2.12) one obtains bounds

$$
\left|\left(A_{i_{1}} \ldots A_{i_{m}} K_{t}^{0}\right)(g)\right| \leq a_{m}^{\prime} t^{-D^{\prime} / 2} e^{\omega t} \int_{G} d h e^{-2 b\left(\left(|g h|^{\prime}\right)^{2}+\left(|h|^{\prime}\right)^{2} / t\right)}
$$

for all $g \in G$ and $t \geq 1$. But the triangle inequality for the modulus $|\cdot|^{\prime}$ implies that

$$
\left(|g h|^{\prime}\right)^{2}+\left(|h|^{\prime}\right)^{2} / 2 t \geq\left(|g|^{\prime}\right)^{2} / 4 t
$$

if $t \geq 1$ and hence by combination (2.14) and (2.15) one finds

$$
\left|\left(A_{i_{1}} \ldots A_{i_{m}} K_{t}^{0}\right)(g)\right| \leq a_{m}^{\prime} t^{-D^{\prime} / 2} e^{\omega t} e^{-b\left(|g|^{\prime}\right)^{2} / 2 t} \int_{G} d h e^{-b\left(|h|^{\prime}\right)^{2} / t}
$$

for all $g \in G$ and $t \geq 1$. Now the integral can be estimated by noting that the Haar measure of the ball $B_{r}=\{g \in G ;|g| \leq r\}$ grows at most exponentially as $r \rightarrow \infty$. Hence

$$
\left|B_{r}^{\prime}\right| \leq\left|B_{r}\right| \leq a e^{\gamma r}
$$

for all $r \geq 1$ and suitable $a, \gamma>0$. Therefore

$$
\begin{aligned}
\int_{G} d h e^{-b\left(|h|^{\prime}\right)^{2} / t} & \leq \sum_{n \geq 0} \int_{n \leq|h|^{\prime} / t^{1 / 2} \leq n+1} d h e^{-b n^{2}} \\
& \leq \sum_{n \geq 0} a e^{\gamma(n+1) t^{1 / 2}} e^{-b n^{2}}
\end{aligned}
$$


for $t \geq 1$. The sum can be straightforwardly estimated and one finds bounds

$$
\int_{G} d h e^{-b\left(|h|^{\prime}\right)^{2} / t} \leq a^{\prime} e^{\gamma^{\prime} t}
$$

for all $t \geq 1$ and suitable $a^{\prime}, \gamma^{\prime}>0$. Combination of (2.16) and (2.18) then gives

$$
\left|\left(A_{i_{1}} \ldots A_{i_{m}} K_{t}^{0}\right)(g)\right| \leq a_{m}^{\prime \prime} t^{-D^{\prime} / 2} e^{\omega^{\prime} t} e^{-b\left(|g|^{\prime}\right)^{2} / 2 t}
$$

for all $t \geq 1$ with $a_{m}^{\prime \prime}, b^{\prime}>0$ and $\omega^{\prime} \geq 0$. But for $t \geq 1$ one has $t^{m / 2} \leq t^{m} \leq m ! \varepsilon^{-m} e^{e t}$ for all $\varepsilon>0$. Therefore (2.19) can be rephrased in the form (2.9). Thus the estimates (2.9) are valid for all $t>0$. 


\section{A priori estimates}

In this section we establish estimates on the left derivatives $A_{1}, \ldots, A_{d^{\prime}}$ which demonstrate that they are small perturbations of the subelliptic operator $H$ on $L_{2}, L_{\hat{2}}$, and $L_{\infty}$. The $L_{2}$-estimates are the direct analogue of the inequalities contained in the $L_{\infty}$-conjecture of Section 1. Subsequently, in Section 6, we discuss extensions of the $L_{\infty}$ results for operators with smooth coefficients.

First consider the $L_{2}$-case with a pure second-order operator $H_{0}$ expressed in the symmetric form (1.20), i.e.,

$$
H_{0}=-\sum_{i, j=1}^{d^{\prime}} A_{i} c_{i j} A_{j}
$$

with $c_{i j} \in L_{\infty ; 1}^{\prime}$. Then for $\varphi \in L_{2 ; 2}^{\prime}$

$$
\left\|A_{i} \varphi\right\|_{2}^{2} \leq \sum_{j=1}^{d^{\prime}}\left\|A_{j} \varphi\right\|_{2}^{2} \leq \lambda_{c}^{-1}\left(\varphi, H_{0} \varphi\right)
$$

Therefore

$$
\left\|A_{i} \varphi\right\|_{2} \leq \varepsilon\left\|H_{0} \varphi\right\|_{2}+\left(4 \varepsilon \lambda_{c}\right)^{-1}\|\varphi\|_{2}
$$

for all $\varepsilon>0$ and $\varphi \in L_{2 ; 2}^{\prime}$. But if

$$
H=H_{0}+\sum_{i=1}^{d^{\prime}} c_{i} A_{i}+c_{0}
$$

one deduces from (3.2) that

$$
N_{2 ; 1}^{\prime}(\varphi) \leq \varepsilon\|H \varphi\|_{2}+\varepsilon\|c\|_{\infty} N_{2 ; 1}^{\prime}(\varphi)+\left(\varepsilon\left\|c_{0}\right\|_{\infty}+\left(4 \varepsilon \lambda_{c}\right)^{-1}\right)\|\varphi\|_{2}
$$

where

$$
\|c\|_{\infty}=\sum_{i=1}^{d^{\prime}}\left\|c_{i}\right\|_{\infty}
$$


Hence for small $\varepsilon$ one can solve these inequalities and deduce that there is an $a>0$ such that

$$
\left\|A_{i} \varphi\right\|_{2} \leq \varepsilon\|H \varphi\|_{2}+a \varepsilon^{-1}\|\varphi\|_{2}
$$

for all $\varphi \in L_{2 ; 2}^{\prime}$, for $i=1, \ldots, d^{\prime}$, and $\varepsilon \in\langle 0,1]$. Note that the value of $a$ depends on the coefficients of $H$ only through the ellipticity constant $\lambda_{c}$, and the norms $\|c\|_{\infty}$, $\left\|c_{0}\right\|_{\infty}$. Alternatively if $H$ is expressed in the form (1.20) with $c_{i j}, c_{i} \in L_{\infty ; 1}^{\prime}$ then the estimates (3.3) are again valid but the value of $a$ depends on $\lambda_{c},\left\|c_{0}\right\|_{\infty}$ and $\sum_{i=1}^{d^{\prime}}\left\|c_{i}\right\|_{\infty ; 1 .}^{\prime}$. This follows because $A_{i} c_{i}=c_{i} A_{i}+\left(A_{i} c_{i}\right)$. Finally if $H$ is expressed in the form (1.17) then (3.3) is still valid but $a$ depends on $\lambda_{c}$ and the norm

$$
\|c\|_{1}^{\prime}=\sum_{\alpha ;|\alpha|=2}\left\|c_{\alpha}\right\|_{\infty ; 1}^{\prime}+\sum_{\alpha ;|\alpha|<2}\left\|c_{\alpha}\right\|_{\infty} .
$$

In fact we have proved the first half of the following result.

Proposition 3.1 If $H$ is a subelliptic operator in the form (1.17) with $c_{\alpha} \in L_{\infty ; 1}^{\prime}$ whenever $|\alpha|=2$ then there are $a, \hat{a}>0$ such that

$$
\begin{aligned}
& N_{2 ; 1}^{\prime}(\varphi) \leq \varepsilon\|H \varphi\|_{2}+a \varepsilon^{-1}\|\varphi\|_{2} \\
& N_{\hat{2} ; 1}^{\prime}(\psi) \leq \varepsilon\|H \psi\|_{\hat{2}}+\hat{a} \varepsilon^{-1}\|\psi\|_{\hat{2}}
\end{aligned}
$$

for all $\varphi \in L_{2 ; 2}^{\prime}, \psi \in L_{\hat{2} ; 2}^{\prime}$ and $\varepsilon \in\langle 0,1]$ where the values of $a$, and $\hat{a}$, depend on the coefficients of $H$ only through the parameters $\lambda_{c}$ and $\|c\|_{1}^{\prime}$.

Proof We have already established the $L_{2}$-estimates but the $L_{\hat{2}}$-estimates are an easy corollary. To deduce the latter first note that for $\psi \in L_{\hat{2} ; 2}^{\prime}$

$$
\begin{aligned}
\left\|A_{i} \psi\right\|_{\hat{2}} & =\left\|\left(\Delta^{-1 / 2} A_{i} \Delta^{1 / 2}\right) \Delta^{-1 / 2} \psi\right\|_{2} \\
& \leq\left\|A_{i} \Delta^{-1 / 2} \psi\right\|_{2}+\left(\left|\beta_{i}\right| / 2\right)\left\|\Delta^{-1 / 2} \psi\right\|_{2}
\end{aligned}
$$

where $\beta_{i}=\left(A_{i} \Delta\right)(e)$. Here we have used $\Delta^{t} A_{i} \Delta^{-t}=A_{i}-t \beta_{i}$. Now applying (3.5) with $H$ replaced by $\Delta^{-1 / 2} H \Delta^{1 / 2}$, which is allowable because the latter is a subelliptic operator of the type under consideration, one obtains bounds

$$
\begin{aligned}
\left\|A_{i} \psi\right\|_{\hat{2}} & \leq \varepsilon\left\|\left(\Delta^{-1 / 2} H \Delta^{1 / 2}\right) \Delta^{-12} \psi\right\|_{2}+a \varepsilon^{-1}\left\|\Delta^{-1 / 2} \psi\right\|_{2}+\left(\left|\beta_{i}\right| / 2\right)\left\|\Delta^{-1 / 2} \psi\right\|_{2} \\
& =\varepsilon\|H \psi\|_{\hat{2}}+\hat{a}_{i} \varepsilon^{-1}\|\psi\|_{\hat{2}}
\end{aligned}
$$


for all $\psi \in-L_{\hat{2} ; 2}^{\prime}$ and $\varepsilon \in\langle 0,1]$ with $\hat{a}_{i}=a+\left|\beta_{i}\right| / 2$. Thus

$$
N_{\hat{2} ; 1}^{\prime}(\psi) \leq \varepsilon\|H \psi\|_{\hat{2}}+\hat{a} \varepsilon^{-1}\|\psi\|_{\hat{2}}
$$

for $\psi \in L_{\hat{2} ; 2}^{\prime}$ and $\varepsilon \in\langle 0,1]$ with $\hat{a}=a+\sup _{1 \leq i \leq d^{\prime}}\left(\left|\beta_{i}\right| / 2\right)$. Again $\hat{a}$ decreases with $\lambda_{c}$ and increases with $\|c\|_{1}^{\prime}$.

Next we aim to prove a version of the estimates (3.5) on $L_{\infty}$. The proof now depends on a number of estimates involving subelliptic operators with constant coefficients and a subelliptic Sobolev inequality.

First, the Sobolev inequality (5.25) in Chapter IV of [Rob1] states that

$$
\|\varphi\|_{\infty} \leq \varepsilon N_{\hat{p} ; 1}^{\prime}(\varphi)+a \varepsilon^{-D^{\prime} /\left(p-D^{\prime}\right)}\|\varphi\|_{\hat{p}}
$$

for all $\varphi \in L_{\hat{p} ; 1}^{\prime}$, and all $p>D^{\prime}$, the local dimension of $G$ with respect to the algebraic basis $a_{1}, \ldots, a_{d^{\prime}}$, and all $\varepsilon \in\langle 0,1]$, where $a$ is a constant depending on $p$.

Secondly, if the $c_{\alpha}$ are constant then there is an $a>0$ such that

$$
\left\|A_{i} x\right\| \leq \varepsilon\|H x\|+a \varepsilon^{-1}\|x\|
$$

for all $i=1, \ldots, d^{\prime}$ and $\varepsilon \in\langle 0,1]$ for a general Banach space representation where the value of $a$ is independent of the particular representation. The proof is straightforward from (2.9). If $H_{0}$ is the (closed) subelliptic operator obtained by setting $c_{\alpha}=0$ for $|\alpha|=0,1$ then there is a corresponding kernel $K^{0}$ satisfying (2.9) and by Laplace transformation

$$
\left\|A_{i}\left(\lambda I+H_{0}\right)^{-1}\right\| \leq a \lambda^{-1 / 2}
$$

for all sufficiently large $\lambda$. Hence

$$
\begin{aligned}
\left\|A_{i} x\right\| & \leq a \lambda^{-1 / 2}\left\|\left(\lambda I+H_{0}\right) x\right\| \\
& \leq a\left(\lambda^{-1 / 2}\left\|H_{0} x\right\|+\lambda^{1 / 2}\|x\|\right) .
\end{aligned}
$$

Setting $\varepsilon=a \lambda^{-1 / 2}$ one obtains (3.8), with $a$ replaced by $a^{2}$, and $H$ replaced by $H_{0}$. But then

$$
\begin{aligned}
\sup _{1 \leq i \leq d^{\prime}}\left\|A_{i} x\right\| & \leq \varepsilon\|H x\|+a \varepsilon^{-1}\|x\|+\varepsilon\left\|\left(H-H_{0}\right) x\right\| \\
& \leq \varepsilon\|H x\|+a \varepsilon^{-1}\|x\|+\varepsilon\left(\sum_{i=1}^{d^{\prime}}\left|c_{i}\right| \cdot\left\|A_{i} x\right\|\right)+\varepsilon\left\|c_{0}\right\|_{\infty}\|x\|
\end{aligned}
$$


which can be solved to give (3.8).

Finally we need a local a priori estimate. If the $c_{\alpha}$ are constant, $c_{\alpha}=0$ for $|\alpha|=0,1, H_{0}$ again denotes the corresponding subelliptic operator, and $\chi \in C_{c}^{\infty}(G)$, then there exists a constant $a$ depending only on $\chi, \lambda_{c}, \sum_{\alpha}\left\|c_{\alpha}\right\|_{\infty}$, and $p$, such that

$$
N_{\hat{p} ; 2}^{\prime}(\chi \varphi) \leq a\left(\left\|H_{0} \varphi\right\|_{\hat{p}}+\|\varphi\|_{\hat{p}}\right)
$$

for all $\varphi \in L_{\hat{p} ; 2}^{\prime}$ and all $p \in\langle 1, \infty\rangle$. This is established in [RoS], Theorem 16(d). The estimate is false in the cases $p=1$ and $p=\infty$ [Orn] [DeLM]. Actually a similar bound holds even in the case $c_{\alpha} \in L_{\infty ; 1}^{\prime}$ by [RoS] but the point is to analyze the dependence of $a$ on the coefficients. Since by finite dimensionality the set of $H_{0}$ with $c_{\alpha}$ constant, $c_{\alpha}=0$ for $|\alpha|=0,1, \lambda_{c} \geq \lambda>0$ and $\Lambda_{c} \leq \mu$ is contained in the convex hull of a finite number of subelliptic operators it is clear that the constant $a$ in (3.10) can be taken to depend on $H_{0}$ only through $\lambda_{c}$ and $\Lambda_{c}$.

We next prove a version of the second estimate for operators with variable coefficients on $L_{\infty}$.

Proposition 3.2 If $H$ is a subelliptic operator of the form (1.17) and $c_{\alpha} \in L_{\infty ; 1}^{\prime}$ whenever $|\alpha|=2$ then for each $\delta>0$ there is an $a_{\delta}>0$ such that

$$
N_{\infty ; 1}^{\prime}(\varphi) \leq \varepsilon\|H \varphi\|_{\infty}+a_{\delta} \varepsilon^{-1-\delta}\|\varphi\|_{\infty}
$$

for all $\varphi \in L_{\infty ; 2}^{\prime}$ and $\varepsilon \in\langle 0,1]$. The value of $a_{\delta}$ depends on the coefficients of $H$ only through the ellipticity constant $\lambda_{c}$ and the norm $\|c\|_{1}^{\prime}$.

The first step in the proof of Proposition 3.2 is a slight refinement of the RothschildStein result.

Proposition 3.3 Let $H$ be a subelliptic operator of the form (1.17) and $\Omega$ a compact subset of $G$. There is an $a>0$ such that

$$
N_{\hat{p} ; 2, \Omega}^{\prime}(\varphi) \leq a\left(\|H \varphi\|_{\hat{p}}+\|\varphi\|_{\hat{p}}\right)
$$

for all $\varphi \in L_{\hat{p} ; 2}^{\prime}$ and $p \in\langle 1, \infty\rangle$ where a depends only on the right translation class of $\Omega, \lambda_{c},\|c\|_{1}^{\prime}$, and $p$. Here

$$
N_{\hat{p} ; 2, \Omega}^{\prime}(\varphi)=\sup _{1 \leq i, j \leq d^{\prime}}\left(\int_{\Omega} d \hat{g}\left|\left(A_{i} A_{j} \varphi\right)(g)\right|^{p}\right)^{1 / p}
$$


Proof We first prove the proposition for the operator $H_{0}$ obtained by setting $c_{\alpha}=0$ for $|\alpha|=0,1$ in $H$. Then for each $h \in G$ let $H_{h}$ be the constant coefficient operator

$$
H_{h}=\sum_{\alpha ;|\alpha|=2} c_{\alpha}(h) A^{\alpha}
$$

But by (3.11) there exists an $a$ independent of $h$ such that

$$
N_{\hat{p} ; 2, \Omega B_{1}}^{\prime}(\varphi) \leq a\left(\left\|H_{h} \varphi\right\|_{\hat{p}}+\|\varphi\|_{\hat{p}}\right)
$$

for all $\varphi \in L_{\hat{p} ; 2}^{\prime}$ where $B_{1}$ is the unit ball in $G$. As each $H_{h}$ commutes with right translation and $\|\cdot\|_{\hat{p}}$ is invariant under right translations $a$ can be taken to be independent of the right translation class of $\Omega$.

Now using the usual Taylor series expansion

$$
\begin{aligned}
L\left(e^{t a_{i}}\right) \varphi-\varphi & =\int_{0}^{t} d s L\left(e^{s a_{i}}\right) A_{i} \varphi \\
& =t A_{i} \varphi+\int_{0}^{t} d s \int_{0}^{s} d u L\left(e^{u a_{i}}\right) A_{i}^{2} \varphi \\
& =t A_{i} \varphi+\int_{0}^{t} d s(t-s) L\left(e^{s a_{i}}\right) A_{i}^{2} \varphi
\end{aligned}
$$

and putting $t=\varepsilon$ one obtains the estimate, similar to (3.8),

$$
\varepsilon\left\|A_{i} \varphi\right\|_{\hat{p}} \leq\left(\varepsilon^{2} / 2\right) e^{\omega \varepsilon}\left\|A_{i}^{2} \varphi\right\|_{\hat{p}}+2\|\varphi\|_{\hat{p}}
$$

where $\omega$ is a constant depending on the modular function $\Delta$. This arises because $L\left(e^{t a_{i}}\right)$ is not isometric on $L_{\hat{p}}$. Combining this with (3.11), we thus obtain an estimate

$$
N_{\hat{p} ; 1, \Omega B_{1}}^{\prime}(\varphi) \leq \varepsilon\left\|H_{h} \varphi\right\|_{\hat{p}}+a \varepsilon^{-1}\|\varphi\|_{\hat{p}}
$$

for $0<\varepsilon \leq 1$, where $a$ is independent of $h$ and the right translation class of $\Omega$.

As the coefficient functions $c_{i j}$ are uniformly continuous there exists a number $r>0$ such that if $\left|g h^{-1}\right| \leq r$ then

$$
\left|c_{i j}(g)-c_{i j}(h)\right| \leq\left(2 a d^{2}\right)^{-1}
$$

where $a$ is the constant in (3.19). We may assume that $r<1$. Now if $\varphi \in L_{\hat{p} ; 2}^{\prime}$, and $\operatorname{supp} \varphi$ is contained in a ball of radius $r$ around $h$ it follows from

$$
\left(H \varphi-H_{h} \varphi\right)(g)=-\sum_{i, j=1}^{d^{\prime}}\left(c_{i j}(g)-c_{i j}(h)\right)\left(A_{i} A_{j} \varphi\right)(g)
$$


and (3.16)-that

$$
\begin{aligned}
\left\|H_{h} \varphi\right\|_{\hat{p}} & \leq\|H \varphi\|_{\hat{p}}+\left(2 a d^{\prime 2}\right)^{-1} d^{2} N_{\hat{p} ; 2}(\varphi) \\
& \leq\|H \varphi\|_{\hat{p}}+\left(\left\|H_{h} \varphi\right\|_{\hat{p}}+\|\varphi\|_{\hat{p}}\right) / 2 .
\end{aligned}
$$

Hence

$$
\left\|H_{h} \varphi\right\|_{\hat{p}} \leq 2\|H \varphi\|_{\hat{p}}+\|\varphi\|_{\hat{p}}
$$

Thus, it follows from (3.16) that

$$
N_{\hat{p} ; 2}^{\prime}(\varphi) \leq a\left(\|H \varphi\|_{\hat{p}}+\|\varphi\|_{\hat{p}}\right)
$$

for some $a>\overline{0}$, provided $\varphi$ is supported in a ball of radius $r$ around some $h \in \Omega$.

Now, as $\Omega$ is compact, there exists a finite partition of unity $\left\{\chi_{k}\right\}$ on $\Omega$ consisting of $C^{\infty}$ functions $\chi_{k}$ with support in a ball of radius $r$ around $g_{k} \in \Omega$ such that $\sum_{k} \chi_{k}(g)=1$ for $g$ in a neighbourhood of $\Omega$. Then

$$
\begin{aligned}
N_{\hat{p} ; 2, \Omega}^{\prime}(\varphi) & =N_{\hat{p} ; 2, \Omega}^{\prime}\left(\sum_{k} \chi_{k} \varphi\right) \\
& \leq \sum_{k} N_{\hat{p} ; 2}^{\prime}\left(\chi_{k} \varphi\right) \\
& \leq a \sum_{k}\left(\left\|H \chi_{k} \varphi\right\|_{\hat{p}}+\left\|\chi_{k} \varphi\right\|_{\hat{p}}\right)
\end{aligned}
$$

But

$$
H \chi_{k} \varphi=\chi_{k} H \varphi+R
$$

where $R$ is a sum of terms consisting of derivatives of $\chi_{k}$, up to second order, multiplied with $A_{i} \varphi, i=1, \ldots, d$ or $\varphi$. Hence using (3.22), (3.21) and (3.19) we obtain estimate (3.13).

If $\Omega$ is replaced by a right translate of $\Omega$ we may replace the $\chi_{k}$ above with the corresponding right translates, and since the $L_{\infty}$-norms of these translates and their left derivatives are the same as before it is clear that $a$ does not depend on the right translation class of $\Omega$.

If now the $c_{\alpha},|\alpha|=0,1$, are nonzero, we see from (3.19) and (3.21) that the first and zeroth order term are dominated locally by the second order term, and hence Proposition 3.3 is valid for a general operator $H$. 
Proof of Proposition 3.2 Let $\varphi \in L_{\infty ; 2}$ and further let $\chi \in C^{\infty}$ be a function on $G$ such that $0 \leq \chi \leq 1, \chi=1$ in a neighbourhood of $e$ and $\chi$ has compact support $\Omega$. Choose a fixed $p>D^{\prime}$. By (3.19) and (3.21) we have

$$
N_{\hat{p} ; 1}^{\prime}(\chi \varphi) \leq \varepsilon\|H \chi \varphi\|_{\hat{p}}+a \varepsilon^{-1}\|\chi \varphi\|_{\hat{p}}
$$

where $a$ only depends on the right translation class, on $\chi, \lambda_{c},\|c\|_{1}^{\prime}$, and $p$.

By the Sobolev estimate (3.7), we also have

$$
N_{\infty ; 1}^{\prime}(\chi \varphi) \leq \varepsilon_{1} N_{\hat{p} ; 2}^{\prime}(\chi \varphi)+a \varepsilon_{1}^{-D^{\prime} /\left(p-D^{\prime}\right)} N_{\hat{p} ; 1}^{\prime}(\chi \varphi)
$$

where $a$ only depends on $p$. On the other hand, if $\psi$ is any function with support in $\Omega$ then

$$
\ldots \quad\|\psi\|_{\hat{p}}=\left(\int_{\Omega} d \hat{g}|\psi(g)|^{p}\right)^{1 / p} \leq\|\psi\|_{\infty}|\Omega|^{1 / p} .
$$

Hence, combining (3.24), (3.25), and (3.13), we obtain

$$
\begin{aligned}
N_{\infty ; 1}^{\prime}(\chi \varphi) \leq & \varepsilon N_{\hat{p} ; 2}^{\prime}(\chi \varphi)+a \varepsilon^{-D^{\prime} /\left(p-D^{\prime}\right)} N_{\hat{p} ; 1}^{\prime}(\chi \varphi) \\
\leq & \varepsilon a^{\prime}\left(\|H \chi \varphi\|_{\hat{p}}+\|\chi \varphi\|_{\hat{p}}\right)+ \\
& a \varepsilon^{-D^{\prime} /\left(p-D^{\prime}\right)}\left(\varepsilon_{1}\|H \chi \varphi\|_{\hat{p}}+a^{\prime \prime} \varepsilon_{1}^{-1}\|\chi \varphi\|_{\hat{p}}\right)
\end{aligned}
$$

Now choosing $\varepsilon_{1}=\varepsilon^{p /\left(p-D^{\prime}\right)}$ this gives bounds

$$
N_{\infty ; 1}^{\prime}(\chi \varphi) \leq b \varepsilon\|H \chi \varphi\|_{\hat{p}}+c \varepsilon^{-1-2 D^{\prime} /\left(p-D^{\prime}\right)}\|\chi \varphi\|_{\hat{p}}
$$

for all $\varepsilon \in\langle 0,1]$. Then combining this with (3.26) one finds bounds

$$
N_{\infty ; 1}^{\prime}(\chi \varphi) \leq \varepsilon\|H \chi \varphi\|_{\infty}+a_{\delta} \varepsilon^{-1-\delta}\|\chi \varphi\|_{\infty}
$$

for each $\delta>0$ and all $\varepsilon \in\langle 0,1]$. Now

$$
A_{i} \chi \varphi=\left(A_{i} \chi\right) \varphi+\chi A_{i} \varphi
$$

and

$$
H \chi \varphi=\chi H \varphi+R
$$

where $R$ is a sum of terms consisting of derivatives of $\chi_{i}$ up to order two multiplied with $A_{i} \varphi, i=1, \ldots, d$ or $\varphi$. Moreover, since

$$
\|\psi\|_{\infty}=\sup _{g \in G}\left\|\chi_{g} \psi\right\|_{\infty}
$$


where $\chi_{g}=R(g) \chi$ and the $a_{\delta}$ in (3.25) only depends on $\chi$ through its right translation class, we finally obtain

$$
N_{\infty ; 1}^{\prime}(\varphi) \leq \varepsilon\|H \varphi\|_{\infty}+a_{\delta} \varepsilon^{-1-\delta}\|\varphi\|_{\infty}
$$

for $0<\varepsilon \leq 1$, and $0<\delta$, where $a_{\delta}$ only depend on $\delta, \lambda_{c}$, and $\|c\|_{1}^{\prime}$. Then, by normclosure one obtains the statement of Proposition 3.2.

In Propositions 3.2 and 3.3 it is not essential that $c_{\alpha} \in L_{\infty ; 1}^{\prime}$ for $|\alpha|=2$. It suffices that the $c_{\alpha}$ for $|\alpha|=2$ are uniformly continuous. Then the constants $a$ depend on $\lambda_{c}$, the norm $\sum_{|\alpha|=0,1,2}\left\|c_{\alpha}\right\|_{\infty}$, and the modulus of continuity of the $c_{\alpha}$ with $|\alpha|=2$, i.e., the smallest positive function $\mu$ such that

$$
\sup _{\alpha ;|\alpha|=2}\left|c_{\alpha}(g)-c_{\alpha}(h)\right| \leq \mu\left(\left|g h^{-1}\right|\right)
$$

for all $g, h \in G$. This is clear from the proofs.

The estimates of Propositions 3.1 and 3.2 will be subsequently useful for the discussion of continuity and boundedness properties of the semigroups generated by subelliptic operators, and their integral kernels, on the $L_{p}$-spaces. The most notable deficiency in the estimates (3.12) is that they are only established for $\delta>0$. They are valid in the limiting case $\delta=0$ if the operator $H$ is strongly elliptic or if the leading coefficients of $H$ are three times differentiable, but in the latter case the value of the parameter $a_{0}$ entering the bounds depends on the norms $\left\|c_{\alpha}\right\|_{\infty ; 3}^{\prime}$ for $\alpha$ with $|\alpha|=2$. This latter result will be established in Section 6 .

Finally we need a version of (3.12) expressed on a Lipschitz space. If $q \in[1, \infty]$ and $\gamma \in\langle 0,1\rangle$ we define the space $\left(L_{\hat{p}}, L_{\hat{p} ; 1}^{\prime}\right)_{q, \gamma}$ to be the interpolation space between $L_{\hat{p}}$ and $L_{\hat{p} ; 1}^{\prime}$ defined by Peetre's $K$-method (see, for example, [BuB] Chapter 3 ). We denote the norm of this space by $\|\cdot\|_{\hat{p}, q, \gamma}^{\prime}$. The spaces are defined by introducing the $K$-function

$$
K_{\varphi}(t)=\inf \left\{\left\|\varphi_{0}\right\|_{\hat{p}}+t\left\|\varphi_{1}\right\|_{\hat{p} ; 1}^{\prime} ; \varphi=\varphi_{0}+\varphi_{1}, \varphi_{1} \in L_{\hat{p} ; 1}^{\prime}\right\}
$$

and then setting

$$
\|\varphi\|_{\hat{p}, q, \gamma}^{\prime}=\|\varphi\|_{\hat{p}}+\left(\int_{0}^{1} d t t^{-1}\left(t^{-\gamma} K_{\varphi}(t)\right)^{q}\right)^{1 / q} .
$$


Then the $C^{1}$-seminorm $N_{\hat{p}, q, \gamma ; 1}^{\prime}$ is defined by

$$
N_{\hat{p}, q, \gamma ; 1}^{\prime}(\varphi)=\sup _{1 \leq i \leq d^{\prime}}\left\|A_{i} \varphi\right\|_{\hat{p}, q, \gamma}^{\prime}
$$

and the $C^{1}$-norm $\|\cdot\|_{\hat{p}, q, \gamma ; 1}^{\prime}$ as the maximum of $\|\varphi\|_{\hat{p}, q, \gamma}^{\prime}$ and $N_{\hat{p}, q, \gamma ; 1}^{\prime}(\varphi)$. The $C^{n}$-norms etc. are defined in an analogous manner.

The Lipschitz spaces $\left(L_{\hat{p}}, L_{\hat{p} ; 1}^{\prime}\right)_{q, \gamma}$ satisfy Sobolev embedding properties similar to those of the $L_{\hat{p}}$-spaces. For example, it follows from (3.7) that

$$
L_{\hat{p} ; 1}^{\prime} \subseteq L_{\infty}
$$

for $p>D^{\prime}$ and hence

$$
L_{\hat{p} ; 2}^{\prime} \subseteq L_{\infty ; 1}^{\prime}
$$

under the same restriction. But then by interpolation one finds

$$
\left(L_{\hat{p}}, L_{\hat{p} ; 1}^{\prime}\right)_{q, \gamma ; 1} \subseteq\left(L_{\infty}, L_{\infty ; 1}^{\prime}\right)_{q, \gamma}
$$

for the $C^{1}$-subspace $\left(L_{\hat{p}}, L_{\hat{p} ; 1}^{\prime}\right)_{q, \gamma ; 1}$ of the interpolation space, i.e., the common domain of the $A_{1}, \ldots, A_{d^{\prime}}$ acting on $\left(L_{\hat{p}}, L_{\hat{p} ; 1}^{\prime}\right)_{q, \gamma}$. The interpolation argument is based on the observations that

$$
\begin{aligned}
\left(L_{\hat{p} ; 1}^{\prime}, L_{\hat{p} ; 2}^{\prime}\right)_{q, \gamma} & =\left(L_{\hat{p}}, L_{\hat{p} ; 2}^{\prime}\right)_{q, \gamma+1} \\
& =\left(L_{\hat{p}}, L_{\hat{p} ; 1}^{\prime}\right)_{q, \gamma ; 1}
\end{aligned}
$$

These identifications were established by Pesenson [Pes] (see also [ElR1] Theorem 2.1).

The proof of the Lipschitz analogue of the Lipschitz inequality (3.7) is a little bit more complicated.

Lemma 3.4 For each $p \in[1, \infty\rangle, q \in[1, \infty]$ and $\gamma \in\langle 0,1\rangle$ there is an a $>0$ such that

$$
\|\varphi\|_{\infty, q, \gamma}^{\prime} \leq \varepsilon N_{\hat{p}, q, \gamma ; 1}^{\prime}(\varphi)+a \varepsilon^{-D^{\prime} /\left(p-D^{\prime}\right)}\|\varphi\|_{\hat{p}, q, \gamma}^{\prime}
$$

for all $\varphi \in\left(L_{\hat{p}}, L_{\hat{p} ; 1}^{\prime}\right)_{q, \gamma}$ and all $\varepsilon \in\langle 0,1]$ whenever $p>D^{\prime}$.

Proof Let $H_{0}=-\sum_{i=1}^{d^{\prime}} A_{i}^{2}+\omega_{0} I$ and let $S^{0}$ denote the semigroup generated on the $L_{\hat{p}}$-spaces by the closure of $H_{0}$. If $\omega_{0}$ is sufficiently large it follows that $\left\|S_{t}^{0}\right\|_{\hat{p} \rightarrow \hat{p}}$ is 
exponentially decreasing as $t \rightarrow \infty$. Moreover, as a consequence of the kernel bounds (2.9) one also has bounds $\left\|A_{i} S_{t}^{0}\right\|_{\hat{p} \rightarrow \hat{p}} \leq a t^{-1 / 2} e^{-\omega t}$ and $\left\|H_{0} S_{t}^{0}\right\|_{\hat{p} \rightarrow \hat{p}} \leq a t^{-1} e^{-\omega t}$ with $\omega>0$.

Next it follows from [ElR1] Theorem 3.2 that $\|\cdot\|_{\hat{p}, q, \gamma}^{\prime}$ is equivalent to the norm

$$
\|\varphi\|_{\hat{p}, q, \gamma}^{S}=\|\varphi\|_{\hat{p}}+\left(\int_{0}^{\infty} d t t^{-1}\left(t^{-\gamma / 2}\left\|\left(I-S_{t}^{0}\right) \varphi\right\|_{\hat{p}}\right)^{q}\right)^{1 / q}
$$

and $\left(L_{\hat{p}}, L_{\hat{p} ; 1}^{\prime}\right)_{q, \gamma}$ consists of those $\varphi \in L_{\hat{p}}$ for which the latter norm is finite. Therefore $\|\varphi\|_{\infty, q, \gamma}^{\prime} \leq c\|\varphi\|_{\infty, q, \gamma}^{S}$. Now the first term $\|\varphi\|_{\infty}$ in the norm $\|\varphi\|_{\infty, q, \gamma}^{S}$ is bounded directly by (3.7),

$$
\begin{aligned}
\|\varphi\|_{\infty} & \leq \varepsilon N_{\hat{p} ; 1}^{\prime}(\varphi)+a \varepsilon^{-D^{\prime} /\left(p-D^{\prime}\right)}\|\varphi\|_{\hat{p}} \\
& \leq \varepsilon N_{\hat{p}, q, \gamma ; 1}^{\prime}(\varphi)+a \varepsilon^{-D^{\prime} /\left(p-D^{\prime}\right)}\|\varphi\|_{\hat{p}, q, \gamma} .
\end{aligned}
$$

The second term can, however, be bounded by (3.7) and the Minkowski inequality as follows;

$$
\begin{gathered}
\left(\int_{0}^{\infty} d t t^{-1}\left(t^{-\gamma / 2}\left\|\left(I-S_{t}^{0}\right) \varphi\right\|_{\infty}\right)^{q}\right)^{1 / q} \leq \varepsilon\left(\int_{0}^{\infty} d t t^{-1}\left(t^{-\gamma / 2} N_{\tilde{p} ; 1}^{\prime}\left(\left(I-S_{t}^{0}\right) \varphi\right)\right)^{q}\right)^{1 / q} \\
+a \varepsilon^{-D^{\prime} /\left(p-D^{\prime}\right)}\left(\int_{0}^{\infty} d t t^{-1}\left(t^{-\gamma / 2}\left\|\left(I-S_{t}^{0}\right) \varphi\right\|_{\hat{p}}\right)^{q}\right)^{1 / q} \cdot
\end{gathered}
$$

Since, by the above observation on equivalent norms, one has bounds

$$
\left(\int_{0}^{\infty} d t t^{-1}\left(t^{-\gamma / 2}\left\|\left(I-S_{t}^{0}\right) \varphi\right\|_{\hat{p}}\right)^{q}\right)^{1 / q} \leq c\|\varphi\|_{\hat{p}, q, \gamma}^{\prime}
$$

it remains to establish that

$$
\left(\int_{0}^{\infty} d t t^{-1}\left(t^{-\gamma / 2} N_{\hat{p} ; 1}^{\prime}\left(\left(I-S_{t}^{0}\right) \varphi\right)\right)^{q}\right)^{1 / q} \leq c^{\prime}\|\varphi\|_{\hat{p}, q, \gamma ; 1}^{\prime} .
$$

But this is achieved by the usual artifices of Lipschitz space technology.

First

$$
\begin{aligned}
\left\|A_{i}\left(I-S_{t}^{0}\right) \varphi\right\|_{\hat{p}} & \leq \int_{t}^{\infty} d s\left\|A_{i} S_{s / 2}^{0}\right\|_{\hat{p} \rightarrow \hat{p}}\left\|H_{0} S_{s / 2}^{0}\right\|_{\hat{p}} \\
& \leq a^{\prime} \int_{t}^{\infty} d s \int_{s}^{\infty} d u s^{-1 / 2}\left\|H_{0}^{2} S_{u / 2}^{0}\right\|_{\hat{p}} .
\end{aligned}
$$

Hence by two applications of the second Hardy inequality ([Rob1] Section II.4) one finds

$$
\left(\int_{0}^{\infty} d t t^{-1}\left(t^{-\gamma / 2} N_{\tilde{p} ; 1}^{\prime}\left(\left(I-S_{t}^{0}\right) \varphi\right)\right)^{q}\right)^{1 / q} \leq a^{\prime \prime}\left(\int_{0}^{\infty} d t t^{-1}\left(t^{2-\gamma^{\prime}}\left\|H_{0}^{2} S_{t}^{0} \varphi\right\|_{\hat{p}}\right)^{q}\right)^{1 / q}
$$


where $\gamma^{\prime}=(1+\gamma) / 2$. But by [ElR1], Theorems 3.1 and 3.2, the right hand side is bounded by the norm of $\left(L_{\hat{p}}, L_{\hat{p} ; 2}^{\prime}\right)_{q, 1+\gamma}$ and by [ElR1] Theorem 2.1 this is equivalent to the norm of $\left(L_{\hat{p}}, L_{\hat{p} ; 1}^{\prime}\right)_{q, \gamma ; 1}$.

The bounds (3.36) now follow from this last observation and hence the desired Sobolev inequalities (3.32) are a consequence of combining (3.33)-(3.36).

The advantage of the Lipschitz spaces is that they have improved regularity properties. In particular if $H_{0}$ is now a general subelliptic operator with constant coefficients then one has bounds

$$
\|\varphi\|_{\hat{p}, q, \gamma ; 2}^{\prime} \leq a\left(\left\|H_{0} \varphi\right\|_{\hat{p}, q, \gamma}^{\prime}+\|\varphi\|_{\hat{p}, q, \gamma}^{\prime}\right)
$$

analogous to the Rothschild-Stein bounds but now valid for all $\varphi$ in the $C^{2}$-subspace of the interpolation space $\left(L_{\hat{p}}, L_{\hat{p} ; 1}^{\prime}\right)_{q, \gamma}$. These bounds follow from [ElR1] Theorem 5.1.III. Specifically one appeals to Statement I of Proposition 4.3 of [ElR1] with $n=1, k=l=2$ and $H=H_{0}$.

Since one now has the Sobolev embedding and the Rothschild-Stein bounds relative to the interpolation spaces one can repeat the above arguments with $L_{\hat{p}}$ replaced by $\left(L_{\hat{p}}, L_{\hat{p} ; 1}^{\prime}\right)_{q, \gamma}$ to obtain the analogue of Proposition 3.2. All previous estimates relative to the norms on $L_{\infty}$ or on $L_{\hat{p}}$ can equally well be made with respect to the norms on the interpolation spaces. Moreover, the proof of the Lipschitz space version of Proposition 3.3 and its application simplify because the Rothschild-Stein bounds hold in the stronger global form.

Corollary 3.5 Let $H$ be a subelliptic operator of the form (1.17) with $c_{\alpha} \in L_{\infty ; 1}^{\prime}$ whenever $|\alpha|=2$. Let $q \in[1, \infty\rangle$ and $\gamma \in\langle 0,1\rangle$. Then for each $\delta>0$ there is an $a_{\delta}>0$ such that

$$
N_{\infty, q, \gamma ; 1}^{\prime}(\varphi) \leq \varepsilon\|H \varphi\|_{\infty, q, \gamma}^{\prime}+a_{\delta} \varepsilon^{-1-\delta}\|\varphi\|_{\infty, q, \gamma}^{\prime}
$$

for all $\varphi \in\left(L_{\infty}, L_{\infty ; 1}^{\prime}\right)_{q, \gamma ; 2}$ and $\varepsilon \in\langle 0,1]$. The value of $a_{\delta}$ depends on the coefficients of $H$ only through the ellipticity constant $\lambda_{c}$ and the norm $\|c\|_{1}^{\prime}$.

The a priori inequalities (3.12) and (3.37) will be of importance in Sections 10 and 11 for the derivation of smoothness properties for the action of semigroups generated by subelliptic operators. 


\section{Resolvent estimates for constant coefficients}

The second key link in the proof of Theorems 1.1 and 1.2 is a variant of the parametrix method developed by Langlands for the analysis of strongly elliptic operators with constant coefficients (see [Rob1], Chapter 1 for details). In this latter context the method is used to represent the resolvent of the operator under examination in terms of the resolvent of a uniformly strongly elliptic operator on $\mathbf{R}^{d}$. Now, however, we attempt to express the resolvent of the subelliptic operator with variable coefficients in terms of the resolvent of a subelliptic operator with constant coefficients. This requires a number of detailed estimates on the latter resolvents and it is these estimates which are the focus of the current section.

Throughout the remainder of this section we consider subelliptic operators $H$ of the form (1.17) but with constant coefficients. Although the estimates we obtain are used principally on $L_{\infty}$ the results of this section are valid whenever $\left(L_{\infty}, G, L\right)$ is replaced by a general isometric representation $(\mathcal{X}, G, U)$ of $G$ on the Banach space $\mathcal{X}$ which is weakly or weakly* continuous. In the first part of the section we only consider the second-order part $H_{0}$ of $H$, i.e.,

$$
H_{0}=\sum_{\alpha ;|\alpha|=2} c_{\alpha} A^{\alpha}
$$

Then we have the estimates

$$
\left|\left(A_{i_{1}} \ldots A_{i_{m}} K_{t}^{0}\right)(g)\right| \leq a_{m} t^{-D^{\prime} / 2} t^{-m / 2} e^{\omega t} e^{-b\left(|g|^{\prime}\right)^{2} / t},
$$

on the heat kernel $K^{0}$ associated with $H_{0}$, analogous to (2.9). (These estimates are presumably also true for the kernel associated with $H$ but this is not necessary in the sequel.) Subsequently we treat $H$ by perturbation theory. We simplify our notation by using $H$ and $H_{0}$ to denote the closed differential operators. Then the essential perturbation estimate is

$$
\left\|H_{1}\left(\lambda I+H_{0}\right)^{-1}\right\| \leq a \lambda^{-1 / 2}
$$

for all large $\lambda$ where

$$
H_{1}=H-H_{0}=\sum_{\alpha ;|\alpha|=0,1} c_{\alpha} A^{\alpha}
$$


and $\|\cdot\|$ denotes the norm on the Banach space $\mathcal{L}(\mathcal{X})$ of bounded operators from $\mathcal{X}$ into $\mathcal{X}$. This estimate follows from (3.9), which is a consequence of (2.9). The value of $a$ depends on the coefficients $c_{\alpha}$ only through $\lambda_{c}$ and the norm $\sum_{|\alpha| \leq 2}\left|c_{\alpha}\right|$.

All the operators we want to estimate are composed of polynomials in the $A_{i}$ and operators of the form

$$
R=\int_{G} d g \hat{R}(g) U(g)
$$

Then it follows from the left-invariance of $d g$ that

$$
A_{i_{1}} \ldots A_{i_{m}} R=\int_{G} d g(-1)^{m}\left(A_{i_{1}} \ldots A_{i_{m}} \hat{R}\right)(g) U(g)
$$

where the $A_{i}$ on the right denote derivatives with respect to the left regular representation. Hence we have the estimate

$$
\left\|A_{i_{1}} \ldots A_{i_{m}} R\right\| \leq\left\|A_{i_{1}} \ldots A_{i_{m}} \hat{R}\right\|_{1}
$$

which will be used throughout this section.

More specifically, the operators we want to estimate are polynomials in $\left(\lambda I+H_{0}\right)^{-1}$ and $A_{i}, i=1, \ldots, d^{\prime}$. To this end we introduce the fractional powers

$$
\left(\lambda I+H_{0}\right)^{-z}=\Gamma(z)^{-1} \int_{0}^{\infty} d t e^{-\lambda t} t^{z-1} S_{t}^{0}
$$

where $S_{t}^{0}=e^{-t H_{0}}$. These are well defined for $\operatorname{Re} \lambda>0$ and $\operatorname{Re} z>0$, and have the properties

$$
\left(\lambda I+H_{0}\right)^{-z_{1}}\left(\lambda I+H_{0}\right)^{-z_{2}}=\left(\lambda I+H_{0}\right)^{-z_{1}-z_{2}},
$$

and $\left(\lambda I+H_{0}\right)^{-1}$ is the usual resolvent.

From now on we assume that $\lambda$ is real. If $\lambda$ is complex, all the estimates we obtain are still valid if $\lambda$ is replaced by $\operatorname{Re} \lambda$ to the right. The case that $\lambda$ is complex will be considered in Section 9.

Lemma 4.1 There exist constants $\eta \geq 0$ and $a_{m}>0$ such that

$$
\left\|A_{i_{1}} A_{i_{2}} \ldots A_{i_{m}}\left(\lambda I+H_{0}\right)^{-z}\right\| \leq a_{m} \frac{\Gamma(\operatorname{Re} z-m / 2)}{|\Gamma(z)|}(\lambda-\eta)^{m / 2-\operatorname{Re} z}
$$

and

$$
\left\|\left(\lambda I+H_{0}\right)^{-z} A_{i_{1}} A_{i_{2}} \ldots A_{i_{m}}\right\| \leq a_{m} \frac{\Gamma(\operatorname{Re} z-m / 2)}{|\Gamma(z)|}(\lambda-\eta)^{m / 2-\operatorname{Re} z}
$$

provided that $\operatorname{Re} z>m / 2, \lambda>\eta$, and $1 \leq i_{k} \leq d^{\prime}$ for $k=1, \ldots, m$. The constant $a_{m}$ is independent of $z$ and $\lambda$, and $\eta$ is independent of $z, \lambda$, and $m$. The values of $a_{m}$ and $\eta$ depend on $\lambda_{c}$ and $\Lambda_{c}$. 
Proof For the first estimate, it follows from (4.7) that it suffices to compute the $L_{1}$-norm of the kernel

$$
R(h)=\Gamma(z)^{-1} \int_{0}^{\infty} d t e^{-\lambda t} t^{z-1}\left(A_{i_{1}} \ldots A_{i_{m}} K_{t}^{0}\right)(h)
$$

But by (2.9),

$$
\left|\left(A_{i_{1}} A_{i_{2}} \ldots A_{i_{m}} K_{t}^{0}\right)(h)\right| \leq a_{m} t^{-D^{\prime} / 2} t^{-m / 2} e^{\omega t} e^{-b|h|^{2} / t}
$$

Hence

$$
\|R\|_{1} \leq c_{m}|\Gamma(z)|^{-1} \int_{0}^{\infty} d t e^{-(\lambda-\omega) t} t^{\operatorname{Re} z-1} t^{-D^{\prime} / 2} t^{-m / 2} \int_{G} d h e^{-b|h|^{2} / t}
$$

We first estimate that

$$
\int_{G} d h e^{-b|h|^{2} / t} \leq c t^{D^{\prime} / 2} e^{\rho t}
$$

for suitable constants $c, \rho>0$. To this end, divide the integration as follows

$$
\begin{aligned}
\int_{G} d h e^{-b|h|^{2} / t} & \leq \sum_{n \geq 0} \int_{n \leq|g|^{\prime} / t^{1 / 2} \leq n+1} d g e^{-b n^{2}} \\
& \leq \sum_{n \geq 0} \int_{|g|^{\prime} \leq(n+1) t^{1 / 2}} d g e^{-b n^{2}}
\end{aligned}
$$

But if $n$ is small, i.e., $(n+1) t^{1 / 2} \leq 1$, then

$$
\left|B_{(n+1) t^{1 / 2}}^{\prime}\right| \leq a\left((n+1) t^{1 / 2}\right)^{D^{\prime}}
$$

for a suitable constant $a$. Since $\sum_{n \geq 0} n^{D^{\prime}} e^{-b n^{2}}$ converges, it follows that the sum over the $n$ such that $(n+1) t^{1 / 2} \leq 1$ is dominated by $a t^{D^{\prime} / 2}$ for a suitable constant $a$. Now consider the $n$ such that $(n+1) t^{1 / 2}>1$. We have

$$
\left|B_{r}^{\prime}\right| \leq\left|B_{r}\right| \leq a e^{\gamma r}
$$

for suitable constants $a$ and $\gamma$ depending only on the Lie group. Thus

$$
\begin{aligned}
\sum_{\substack{n \geq 0 \\
(n+1) t^{1 / 2} \geq 1}}\left|B_{(n+1) t^{1 / 2}}^{\prime}\right| e^{-b n^{2}} & \leq \sum_{\substack{n \geq 0 \\
(n+1) t^{1 / 2} \geq 1}} a e^{\gamma(n+1) t^{1 / 2}} e^{-b n^{2}} \\
& \leq a \int_{t^{-1 / 2}}^{\infty} d x e^{\gamma(x+1) t^{1 / 2}} e^{-b(x-1)^{2}} \\
& \leq a e^{\rho t}
\end{aligned}
$$


for a suitable $\rho$. Note also that as $t \rightarrow 0$ then the integral above tends to zero faster than any power of $t$. Combining this with the estimate for the small $n$ sum we obtain (4.15).

Inserting (4.15) into (4.14) we obtain

$$
\|R\|_{1} \leq a_{m} a|\Gamma(z)|^{-1} \int_{0}^{\infty} d t e^{-(\lambda-(\omega+\rho)) t} t^{\operatorname{Re} z-1} t^{-m / 2} .
$$

The change of variable $u=(\lambda-\eta) t$, where $\eta=\omega+\rho$, gives

$$
\|R\|_{1} \leq a_{m} a|\Gamma(z)|^{-1}(\lambda-\eta)^{m / 2-\operatorname{Re} z} \int_{0}^{\infty} d u e^{-u} u^{\operatorname{Re} z-m / 2-1} .
$$

The last integral converges provided $\operatorname{Re} z-m / 2>0$, and we obtain

$$
\|R\|_{1} \leq a_{m} a|\Gamma(z)|^{-1} \Gamma(\operatorname{Re} z-m / 2)(\lambda-\eta)^{m / 2-\operatorname{Re} z} .
$$

This proves the first estimate in Lemma 4.1.

To prove the second estimate, let $\mathcal{F}$ be the dual or predual of $\mathcal{X}$ according to whether $U$ is a weakly continuous or weakly* continuous isometric representation of G. The formal adjoint of $H_{0}$ has exactly the same form as $H_{0}$, and is a pregenerator on $\mathcal{F}$, and hence we have the estimate (4.10) when $\|\cdot\|_{\mathcal{X} \rightarrow \mathcal{X}}$ is replaced by $\|\cdot\|_{\mathcal{F} \rightarrow \mathcal{F} \text {. }}$ But the operator

$$
\left(\lambda I+H_{0}\right)^{-z} A_{i_{1}} \ldots A_{i_{m}}
$$

on $\mathcal{X}$, is the adjoint of the operator

$$
(-1)^{m} A_{i_{m}} \ldots A_{i_{1}}\left(\lambda I+H_{0}\right)^{-z}
$$

on $\mathcal{F}$. Hence the estimate (4.11) follows by duality.

The next lemma involves slightly more complicated products of operators and the singular behaviour of $\left(\lambda I+H_{0}\right)^{-z}$ at $\operatorname{Re} z=0$ is more critical. Note that in the representation (4.8) for the resolvents the integral diverges for $\operatorname{Re} z=0$ and in addition $\Gamma$ has a pole at $z=0$. This is the reason for introducing the small positive constant $\varepsilon$ in the following statement.

Lemma 4.2 There exist constants $\eta \geq 0, a_{n} \geq 0$ such that for each $\beta>m / 2$ with $0<\varepsilon<\beta-m / 2$, and each $\alpha$ with $\varepsilon \leq \alpha \leq \beta-\varepsilon$ one has

$$
\begin{aligned}
& \left\|\left(\lambda_{1} I+H_{0}\right)^{-\alpha} A_{i_{1}} \ldots A_{i_{m}}\left(\lambda_{2} I+H_{0}\right)^{-\beta+\alpha}\right\| \\
& \quad \leq a_{m} \frac{\Gamma(\beta-\varepsilon-m / 2) \Gamma(\varepsilon)}{\Gamma(\alpha) \Gamma(\beta-\alpha)}\left(\lambda_{1}-\eta\right)^{\gamma_{1}}\left(\lambda_{2}-\eta\right)^{\gamma_{2}}
\end{aligned}
$$


where

$$
\gamma_{1}=(-\varepsilon(\beta-\varepsilon-\alpha)+(-\beta+\varepsilon+m / 2)(\alpha-\varepsilon)) /(\beta-2 \varepsilon)
$$

and

$$
\gamma_{2}=((-\beta+\varepsilon+m / 2)(\beta+\varepsilon-\alpha)-\varepsilon(\alpha-\varepsilon)) /(\beta-2 \varepsilon)
$$

provided that $\lambda_{1}, \lambda_{2}>\eta$ and $1 \leq i_{k} \leq d^{\prime}$ for $k=1, \ldots, m$. Here $a_{m}$ and $\eta$ are independent of $\alpha, \beta, \varepsilon$ and $\eta$ is independent of $m$.

Remark If $\lambda_{1}=\lambda_{2}=\lambda$ then the $\lambda$-dependent factor in the bound (4.19) is equal to $(\lambda-\eta)^{m / 2-\beta}$.

Proof Let $\mathcal{F}$ be the dual or predual of $\mathcal{X}$, according to whether $U$ is weakly, or weakly*, continuous. By an extension of the argument used to derive (3.8) from (2.9) one deduces that there is a constant $a_{m}$ such that

$$
\left\|A_{i_{1}} \ldots A_{i_{m}} x\right\| \leq a_{m}\left(\left\|H_{0}^{m+1} x\right\|+\|x\|\right)
$$

for $x \in \mathcal{X}$, or $x \in \mathcal{F}$, where $1 \leq i_{k} \leq d^{\prime}$. Now, assume $x \in \mathcal{X}_{2(m+1)}$ and $y \in \mathcal{F}$ and consider the function

$$
\begin{aligned}
f(z) & =\Gamma(z) \Gamma(\beta-z)\left(y,\left(\lambda_{1} I+H\right)^{-z} A_{i_{1}} \ldots A_{i_{m}}\left(\lambda_{2} I+H\right)^{-\beta+z} x\right) \\
& =\Gamma(z) \Gamma(\beta-z)\left(\left(\lambda_{1} I+H_{0}^{\dagger}\right)^{-z} y, A_{i_{1}} \ldots A_{i_{m}}\left(\lambda_{2} I+H_{0}^{\dagger}\right)^{-\beta+z} x\right)
\end{aligned}
$$

where we have used $H_{0}^{*}=H_{0}^{\dagger}\left(=H_{0}\right)$. It follows readily from (4.10) for $m=0$ and (4.20) that $f$ is well defined for all $z$ with $0<\operatorname{Re} z<\beta$. More specifically, we have the estimate

$$
\begin{aligned}
|f(z)| \leq|\Gamma(z)| \cdot|\Gamma(\beta-z)| \cdot\left\|\left(\lambda_{1} I+H_{0}^{\dagger}\right)^{-z} y\right\|_{\mathcal{F}} \cdot \\
\cdot\left\|A_{i_{1}} \ldots A_{i_{m}}\left(\lambda_{2} I+H\right)^{-\beta+z} x\right\|_{\mathcal{X}} \\
\leq|\Gamma(z)| \cdot|\Gamma(\beta-z)| \cdot a_{m} \frac{\Gamma(\operatorname{Re} z)}{|\Gamma(z)|}\left(\lambda_{1}-\eta\right)^{-\operatorname{Re} z}\|y\|_{\mathcal{F}} \cdot \\
\cdot a_{m}\left(\left\|H_{0}^{m+1}\left(\lambda_{2} I+H_{0}\right)^{-\beta+z} x\right\|_{\mathcal{X}}+\left\|\left(\lambda_{2} I+H_{0}\right)^{-\beta+z} x\right\|_{\mathcal{X}}\right) .
\end{aligned}
$$

Furthermore, as $H_{0}$ commutes with $\left(\lambda I+H_{0}\right)^{-\beta+z}$, we obtain

$$
\begin{gathered}
|f(z)| \leq a_{m}^{3} \Gamma(\operatorname{Re} z) \Gamma(\beta-\operatorname{Re} z)\left(\lambda_{1}-\eta\right)^{-\operatorname{Re} z}\left(\lambda_{2}-\eta\right)^{-\beta+\operatorname{Re} z}\|y\|_{\mathcal{F}} \\
\cdot\left(\left\|H_{0}^{m+1} x\right\|_{\mathcal{F}}+\|x\|_{\mathcal{F}}\right) .
\end{gathered}
$$


Thus $f$ is well defined, and by similarly estimating the formal derivative of $f$ with respect to $z$ one sees that $f$ is analytic in the strip $0<\operatorname{Re} z<\beta$. Also, as $\alpha \mapsto \Gamma(\alpha)$ is bounded in $\varepsilon<\alpha<\beta-\varepsilon$, one sees from (4.22) that $f$ is bounded in the strip $\varepsilon \leq \operatorname{Re} z \leq \beta-\varepsilon$. It then follows from Hadamard's three line theorem that

$$
|f(z)| \leq M_{\beta-\varepsilon}^{(\operatorname{Re} z-\varepsilon) /(\beta-2 \varepsilon)} M_{\varepsilon}^{(\beta-\varepsilon-\operatorname{Re} z) /(\beta-2 \varepsilon)}
$$

where

$$
M_{\delta}=\sup \{|f(z)| ; \operatorname{Re} z=\delta\} .
$$

Thus (4.19) follows once we can establish appropriate estimates on $M_{\varepsilon}$ and $M_{\beta-\varepsilon}$. If Re $z=\varepsilon$, i.e., $z=\varepsilon+i \xi$ where $\xi \in \mathrm{R}$, we have from Lemma 4.1 with $A_{i_{1}} \ldots A_{i_{m}}=A^{m}$,

$$
\begin{aligned}
|f(\varepsilon+i \xi)| \leq & |\Gamma(\varepsilon+i \xi)| \cdot|\Gamma(\beta-\varepsilon-i \xi)| \cdot \\
& \cdot\|y\|_{\mathcal{F}}\left\|\left(\lambda_{1} I+H_{0}\right)^{-\varepsilon-i \xi}\right\|_{\mathcal{X} \rightarrow \mathcal{X}}\left\|A^{m}\left(\lambda_{2} I+H_{0}\right)^{-\beta+\varepsilon+i \xi}\right\|_{\mathcal{X} \rightarrow \mathcal{X}}\|x\|_{\mathcal{X}} \\
\leq & a_{m}^{2} \Gamma(\varepsilon)\left(\lambda_{1}-\eta\right)^{-\varepsilon} \Gamma(\beta-\varepsilon-m / 2)\left(\lambda_{2}-\eta\right)^{-\beta+\varepsilon+m / 2} \cdot\|y\|_{\mathcal{F}}\|x\|_{\mathcal{X}} \\
= & M_{\varepsilon} .
\end{aligned}
$$

Similarly, when $\operatorname{Re} z=\beta-\varepsilon$, one can use the splitting

$$
\left(\lambda_{1} I+H_{0}\right)^{-(\beta-\varepsilon)-i \xi} A^{m}\left(\lambda_{2} I+H_{0}\right)^{-\varepsilon+i \xi}=\left(\left(\lambda_{1} I+H_{0}\right)^{-(\beta-\varepsilon)-i \xi} A^{m}\right)\left(\lambda_{2} I+H_{0}\right)^{-\varepsilon+i \xi}
$$

and (4.11) to obtain a bound with

$$
M_{\beta-\varepsilon}=a_{m}^{2} \Gamma(\varepsilon) \Gamma(\beta-\varepsilon-m / 2)\left(\lambda_{1} I-\eta\right)^{-\beta+\varepsilon+m / 2}\left(\lambda_{2} I-\eta\right)^{-\varepsilon}\|y\|_{\mathcal{F}}\|x\|_{\mathcal{X}} .
$$

Thus, by the three line theorem,

$$
\begin{aligned}
|f(z)| \leq & a_{m}^{2} \Gamma(\varepsilon) \Gamma(\beta-\varepsilon-m / 2)\|y\|_{\mathcal{F}}\|x\|_{\mathcal{X}} . \\
& \cdot\left(\left(\lambda_{1}-\eta\right)^{-\varepsilon}\left(\lambda_{2}-\eta\right)^{-\beta+\varepsilon+m / 2}\right)^{(\beta-\varepsilon-\operatorname{Re} z) /(\beta-2 \varepsilon)} . \\
& \cdot\left(\left(\lambda_{1}-\eta\right)^{-\beta+\varepsilon+m / 2}\left(\lambda_{2}-\eta\right)^{-\varepsilon}\right)^{(\operatorname{Re} z-\varepsilon) /(\beta-2 \varepsilon)}
\end{aligned}
$$

and specializing to $z=\alpha \in \mathbf{R}$ this implies (4.19).

We first use Lemma 4.2 in the special case $\lambda_{1}=\lambda_{2}=\lambda$, and the estimate simplifies to

$$
\begin{aligned}
\|\left(\lambda I+H_{0}\right)^{-\alpha} A_{i_{1}} \ldots A_{i_{m}}(\lambda I & \left.+H_{0}\right)^{-\beta+\alpha} \| \\
& \leq a_{m} \frac{\Gamma(\beta-\varepsilon-m / 2) \Gamma(\varepsilon)}{\Gamma(\alpha) \Gamma(\beta-\alpha)}(\lambda-\eta)^{m / 2-\beta} .
\end{aligned}
$$

This is instrumental in the proof of the next result. 
Lemma 4.3 There exist constants $a, \lambda_{0}>0$ such that

$$
\begin{gathered}
\left\|\left(\lambda I+H_{0}\right)^{-1}\right\| \leq a \lambda^{-1} \\
\left\|\left(\lambda I+H_{0}\right)^{-1} A_{i_{1}} A_{i_{2}}\left(\lambda I+H_{0}\right)^{-1}\right\| \leq a \lambda^{-1} \\
\left\|\left(\lambda I+H_{0}\right)^{-1} A_{i_{1}} A_{i_{2}}\left(\lambda I+H_{0}\right)^{-1} A_{i_{3}} A_{i_{4}}\left(\lambda I+H_{0}\right)^{-1}\right\| \leq a \lambda^{-1} \\
\left\|A_{i_{1}}\left(\lambda I+H_{0}\right)^{-1}\right\| \leq a \lambda^{-1 / 2} \\
\left\|A_{i_{1}}\left(\lambda I+H_{0}\right)^{-1} A_{i_{2}} A_{i_{3}}\left(\lambda I+H_{0}\right)^{-1}\right\| \leq a \lambda^{-1 / 2}
\end{gathered}
$$

for all $\lambda>\lambda_{0}$ and $1 \leq i_{k} \leq d^{\prime}$.

Remark One may obtain many other similar estimates for monomials in the $A_{i}$ and the resolvents $\left(\lambda I+H_{0}\right)^{-1}$ but these are the ones needed in the sequel. Again the values of $a$ and $\lambda_{0}$ depend on the coefficients of $H_{0}$ only through the ellipticity parameters $\lambda_{c}$ and $\Lambda_{c}$.

Proof The first and fourth estimates are special cases of Lemma 4.1, whilst the second is a special case of (4.25), or it follows from Lemma 4.1 by using the splitting

$$
\left(\lambda I+H_{0}\right)^{-1} A_{i_{1}} A_{i_{2}}\left(\lambda I+H_{0}\right)^{-1}=\left(\left(\lambda I+H_{0}\right)^{-1} A_{i_{1}}\right)\left(A_{i_{2}}\left(\lambda I+H_{0}\right)^{-1}\right) .
$$

However, to prove the third and fifth estimates we make essential use of (4.25):

$$
\begin{aligned}
& \left\|\left(\lambda I+H_{0}\right)^{-1} A_{i_{1}} A_{i_{2}}\left(\lambda I+H_{0}\right)^{-1} A_{i_{3}} A_{i_{4}}\left(\lambda I+H_{0}\right)^{-1}\right\| \\
& \quad \leq\left\|\left(\lambda I+H_{0}\right)^{-1} A_{i_{1}} A_{i_{2}}\left(\lambda I+H_{0}\right)^{-1 / 2}\right\| \cdot\left\|\left(\lambda I+H_{0}\right)^{-1 / 2} A_{i_{3}} A_{i_{4}}\left(\lambda I+H_{0}\right)^{-1}\right\| \\
& \quad \leq a \lambda^{-1 / 2} \cdot \lambda^{-1 / 2}=a \lambda^{-1}
\end{aligned}
$$

and

$$
\begin{aligned}
& \left\|A_{i_{1}}\left(\lambda I+H_{0}\right)^{-1} A_{i_{2}} A_{i_{3}}\left(\lambda I+H_{0}\right)^{-1}\right\| \\
& \quad \leq\left\|A_{i_{1}}\left(\lambda I+H_{0}\right)^{-2 / 3}\right\| \cdot\left\|\left(\lambda I+H_{0}\right)^{-1 / 3} A_{i_{2}} A_{i_{3}}\left(\lambda I+H_{0}\right)^{-1}\right\| \\
& \quad \leq a \lambda^{-2 / 3+1 / 2} \lambda^{-4 / 3+2 / 2}=a \lambda^{-1 / 2} .
\end{aligned}
$$

We are now going to prove Lemma 4.3 for a general $H$ of the form (4.1) by using perturbation theory. Write

$$
H=H_{0}+H_{1}
$$


where $H_{0}$ is the pure second order part of $H$, given by (4.2) and $H_{1}$ is the lower order part

$$
H_{1}=\sum_{\alpha ;|\alpha|=0,1} c_{\alpha} A^{\alpha}
$$

Then, using (3.8), we have the estimate

$$
\left\|H_{1} x\right\| \leq \varepsilon\left\|H_{0} x\right\|+a \varepsilon^{-1}\|x\|
$$

for all $\varepsilon \in\langle 0,1]$. Applying this with $x$ replaced by $\left(\lambda I+H_{0}\right)^{-1} x$, and using (4.10), we obtain the bounds

$$
\begin{aligned}
\left\|H_{1}\left(\lambda I+H_{0}\right)^{-1} x\right\| & \leq \varepsilon\|x\|+\left(\varepsilon \lambda+a \varepsilon^{-1}\right)\left\|\left(\lambda I+H_{0}\right)^{-1} x\right\| \\
& \leq\left(\varepsilon+a\left(\varepsilon \lambda+\varepsilon^{-1}\right) \lambda^{-1}\right)\|x\|
\end{aligned}
$$

for a suitable new constant $a$ and all large $\lambda$. Thus for each $\varepsilon>0$ there is a $\lambda_{\varepsilon}>0$ such that

$$
\left\|H_{1}\left(\lambda I+H_{0}\right)^{-1}\right\|<\varepsilon
$$

for all $\lambda>\lambda_{\varepsilon}$. Then by duality, one obtains

$$
\left\|\left(\lambda I+H_{0}\right)^{-1} H_{1}\right\|<\varepsilon
$$

for all $\lambda>\lambda_{\varepsilon}$.

Next, using (4.34) and (4.35) with $\varepsilon<1$, it is clear that we can write

$$
\begin{aligned}
(\lambda I+H)^{-1} & =\left(\lambda I+H_{0}\right)^{-1} K_{R, \lambda} \\
& =K_{L, \lambda}\left(\lambda I+H_{0}\right)^{-1}
\end{aligned}
$$

where

$$
\begin{aligned}
K_{R, \lambda} & =\left(I+H_{1}\left(\lambda I+H_{0}\right)^{-1}\right)^{-1} \\
& =\sum_{n=0}^{\infty}\left(-H_{1}\left(\lambda I+H_{0}\right)^{-1}\right)^{n}
\end{aligned}
$$

and

$$
\begin{aligned}
K_{L, \lambda} & =\left(I+\left(\lambda I+H_{0}\right)^{-1} H_{1}\right)^{-1} \\
& =\sum_{n=0}^{\infty}\left(-\left(\lambda I+H_{0}\right)^{-1} H_{1}\right)^{n} .
\end{aligned}
$$


Thus $K_{R, \lambda}$ and $K_{L, \lambda}$ are uniformly bounded in $\lambda$ for large $\lambda$.

Hence, if we replace $H_{0}$ by $H$ in Lemma 4.3, the estimates (4.26), (4.27) and (4.29) follow immediately from the corresponding estimates on $H_{0}$ and the boundedness of $K_{R, \lambda}$ and $K_{L, \lambda}$ in (4.35). Moreover, to prove (4.28) and (4.30) it suffices to show that

$$
\left\|A(\lambda I+H)^{-\alpha}\right\| \leq \mathrm{O}\left(\lambda^{1 / 2-\alpha}\right)
$$

for $1 / 2<\alpha \leq 1$, and

$$
\left\|(\lambda I+H)^{-\alpha} A^{2}\left(\lambda I+H_{0}\right)^{-1}\right\| \leq \mathrm{O}\left(\lambda^{-\alpha}\right)
$$

for $0<\alpha<1$, where $\lambda$ is assumed to be large in both estimates and $A^{m}$ denotes a monomial of order $m$ in $A_{1}, \ldots, A_{d^{\prime}}$. To this end we use the representation

$$
(\lambda I+H)^{-\alpha}=(\sin \alpha \pi / \pi) \int_{0}^{\infty} d \mu \mu^{-\alpha}((\lambda+\mu) I+H)^{-1}
$$

which is valid for $0<\alpha<1$, [Yos]. In view of (4.36) and this representation, all we need to establish (4.39) and (4.40) is that

$$
\int_{0}^{\infty} d \mu \mu^{-\alpha}\left\|A\left((\lambda+\mu) I+H_{0}\right)^{-1}\right\| \leq \mathrm{O}\left(\lambda^{1 / 2-\alpha}\right)
$$

for $1 / 2<\alpha \leq 1$ and that

$$
\int_{0}^{\infty} d \mu \mu^{-\alpha}\left\|\left((\lambda+\mu) I+H_{0}\right)^{-1} A^{2}\left(\lambda I+H_{0}\right)^{-1}\right\| \leq \mathrm{O}\left(\lambda^{-\alpha}\right)
$$

for $0<\alpha<1$ respectively. But by (4.29), the integral in (4.42) is dominated by a multiple of

$$
\begin{aligned}
\int_{0}^{\infty} d \mu \mu^{-\alpha}(\lambda+\mu)^{-1 / 2} & =\int_{0}^{\infty} d \mu \lambda(\mu \lambda)^{-\alpha}(1+\mu)^{-1 / 2} \lambda^{-1 / 2} \\
& =\lambda^{1 / 2-\alpha} \int_{0}^{\infty} d \mu \mu^{-\alpha}(1+\mu)^{-1 / 2}
\end{aligned}
$$

As for (4.43), we estimate

$$
\begin{aligned}
& \mu^{-\alpha}\left\|\left((\lambda+\mu) I+H_{0}\right)^{-1} A^{2}\left(\lambda I+H_{0}\right)^{-1}\right\| \\
& \quad \leq \mu^{-\alpha}\left\|\left((\lambda+\mu) I+H_{0}\right)^{-1+\alpha / 2}\right\| \cdot\left\|\left((\lambda+\mu) I+H_{0}\right)^{-\alpha / 2} A^{2}\left(\lambda I+H_{0}\right)^{-1}\right\| \\
& \quad \leq \mu^{-\alpha} \mathrm{O}\left((\lambda+\mu)^{-1+\alpha / 2}\right) \mathrm{O}\left((\lambda+\mu)^{\gamma_{1}} \lambda^{\gamma_{2}}\right)
\end{aligned}
$$

for all $\mu \geq 0$, and all large $\lambda$, where

$$
\begin{aligned}
& \gamma_{1}=-\left(\varepsilon(1-\varepsilon)+(\alpha / 2-\varepsilon)^{2}\right) /(\alpha / 2+1-2 \varepsilon), \\
& \gamma_{2}=-(\alpha / 2-\varepsilon) /(\alpha / 2+1-2 \varepsilon)
\end{aligned}
$$


and $0<\varepsilon \leq \alpha / 2$. Here we have used Lemma 4.2. It follows that the integral (4.43) converges and, by a change of variable, as above, one deduces that

$\int_{0}^{\infty} d \mu \mu^{-\alpha}(\lambda+\mu)^{-1+\alpha / 2}(\lambda+\mu)^{\gamma_{1}} \lambda^{\gamma_{2}}=\lambda^{-\alpha / 2+\gamma_{1}+\gamma_{2}} \int_{0}^{\infty} d \mu \mu^{-\alpha}(1+\mu)^{-1+\alpha / 2+\gamma_{1}}$.

But $\gamma_{1}+\gamma_{2}=-\alpha / 2$ and hence the integral is given by a multiple of $\lambda^{-\alpha}$. This establishes (4.42) and we have proved the following generalization of Lemma 4.2.

Lemma 4.4 If $H$ is a subelliptic operator with constant coefficients there exist $a, \lambda_{0}>0$ such that

$$
\begin{gathered}
\left\|(\lambda I+H)^{-1}\right\| \leq a \lambda^{-1} \\
\left\|(\lambda I+H)^{-1} A_{i_{1}} A_{i_{2}}(\lambda I+H)^{-1}\right\| \leq a \lambda^{-1} \\
\left\|(\lambda I+H)^{-1} A_{i_{1}} A_{i_{2}}(\lambda I+H)^{-1} A_{i_{3}} A_{i 4}(\lambda I+H)^{-1}\right\| \leq a \lambda^{-1} \\
\left\|A_{i_{1}}(\lambda I+H)^{-1}\right\| \leq a \lambda^{-1 / 2} \\
\left\|A_{i_{1}}(\lambda I+H)^{-1} A_{i_{2}} A_{i_{3}}(\lambda I+H)^{-1}\right\| \leq a \lambda^{-1 / 2}
\end{gathered}
$$

for all $\lambda>\lambda_{0}$ and $1 \leq i_{k} \leq d^{\prime}$. The values of $a$ and $\lambda_{0}$ depend on the coefficients of $H$ through $\lambda_{c}$ and the norm $\sum_{\alpha}\left|c_{\alpha}\right|$.

The estimates of this lemma are essential for the subsequent discussion of parametrices for operators with smooth coefficients. 


\section{The parametrix method}

In this section we begin the analysis of the subelliptic operators (1.17) with variable coefficients. The method we develop requires the coefficients $c_{\alpha}$ to be smooth, e.g., $c_{\alpha} \in L_{\infty ; 2}^{\prime}$ or $L_{\infty ; 3}^{\prime}$, but these assumptions can be weakened by subsequent additional arguments given in Sections 10 and 11.

First, for each $h \in C$ introduce the constant coefficient operator $H_{h}$ by

$$
\left(H_{h} \varphi\right)(g)=\sum_{\alpha ;|\alpha| \leq 2} c_{\alpha}(h)\left(A^{\alpha} \varphi\right)(g)
$$

and let $R_{\lambda}(\cdot ; h)$ be the fundamental solution of the operator $\lambda I+H_{h}$. Thus $R_{\lambda}(\cdot ; h)$, for each fixed $h$, is the kernel of the resolvent $\left(\lambda I+H_{h}\right)^{-1}$ considered in Section 4. Now define the (right) parametrix $R_{\lambda}$ as the operator

$$
\begin{aligned}
\left(R_{\lambda} \varphi\right)(g) & =\int_{G} d h \psi(h) R_{\lambda}(h ; g)(L(h) \varphi)(g) \\
& =\int_{G} d \hat{h} \psi\left(g h^{-1}\right) R_{\lambda}\left(g h^{-1} ; g\right) \varphi(h)
\end{aligned}
$$

where $\psi$ is a function in $C_{c}^{\infty}(G)$ with $0 \leq \psi \leq 1$ and $\psi(e)=1$. The parametrix can be thought of as an approximation to the resolvent $(\lambda I+H)^{-1}$ of $H$, whose existence has yet to be established. Thus we are interested in analyzing $R_{\lambda}$ as an approximate right inverse of $(\lambda I+H)$. Hence we introduce the remainders $Q_{\lambda}$ by

$$
(\lambda I+H) R_{\lambda}=I+Q_{\lambda}
$$

and we aim to show that these are small, at least for large $\lambda$.

In order to analyze $R_{\lambda}$ and $Q_{\lambda}$ we need some basic properties of the kernel $R_{\lambda}(\cdot ; \cdot)$. Note first that if $\mathrm{R}$ is any operator from $L_{\infty}$ into $L_{\infty}$ given by a kernel, i.e.,

$$
(R \varphi)(g)=\int_{G} d h R(h ; g) \varphi\left(h^{-1} g\right)
$$

then it follows easily that

$$
\|R\|_{\infty \rightarrow \infty}=\underset{g \in G}{\operatorname{ess} \sup } \int_{G} d h|R(h ; g)| .
$$


But if $R_{k},-k \in G$, is the operator on $L_{\infty}$ defined by

$$
\left(R_{k} \varphi\right)(g)=\int_{G} d h R(h ; k) \varphi\left(h^{-1} g\right)
$$

then

$$
\left\|R_{k}\right\|_{\infty \rightarrow \infty}=\int_{G} d h|R(h ; k)|
$$

and hence

$$
\|R\|_{\infty \rightarrow \infty}=\operatorname{esssup}_{k \in G}\left\|R_{k}\right\|_{\infty \rightarrow \infty}
$$

Thus we have obtained the following estimate.

Lemma 5.1 Let $H_{g}$ be the constant coefficient operator defined by (5.1). Then the parametrix $R_{\lambda}$ of $H$ satisfies

$$
\left\|R_{\lambda}\right\|_{\infty \rightarrow \infty} \leq \underset{g \in G}{\operatorname{ess} \sup _{0}}\left\|\left(\lambda I+H_{g}\right)^{-1}\right\|_{\infty \rightarrow \infty} .
$$

Now in Lemma 4.4 we estimated that

$$
\left\|\left(\lambda I+H_{g}\right)^{-1}\right\|_{\infty \rightarrow \infty} \leq a \lambda^{-1}
$$

for all $\lambda>\lambda_{0}$ where the values of $a$ and $\lambda_{0}$ depend only on the coefficients of $H_{g}$ through the lower ellipticity constant

$$
\inf \left\{-\sum_{\alpha ;|\alpha|=2} c_{\alpha}(g) \xi^{2} ; \xi \in \mathbf{R}^{d_{1}},|\xi|=1\right\}
$$

and the norm

$$
\sum_{\alpha ;|\alpha| \leq 2}\left|c_{\alpha}(g)\right|
$$

Thus because of the general subellipticity assumption and as $c_{\alpha} \in L_{\infty}$ one can choose $a$, and $\lambda_{0}$, such that these estimates are valid uniformly in $g$.

Lemma 5.2 There exist $a, \lambda_{0}>0$ such that

$$
\left\|R_{\lambda}\right\|_{\infty \rightarrow \infty} \leq a \lambda^{-1}
$$

for all $\lambda>\lambda_{0}$. The values of $a$, and $\lambda_{0}$, depend on the coefficients of $H$ through the ellipticity constant $\lambda_{c}$ and the norm

$$
\|c\|=\sum_{\alpha ;|\alpha| \leq 2}\left\|c_{\alpha}\right\|_{\infty}
$$


In order to show that $R_{\lambda}$ maps $C_{0}(G)$ into $C_{0}(G)$, and not merely $L_{\infty}$ into $L_{\infty}$, and also to estimate the remainder $Q_{\lambda}$ we need estimates on the derivatives of $R_{\lambda}(\cdot ; \cdot)$ with respect to both variables. We use $A_{i}$ to denote the left derivative in the direction $a_{i}$ in the first variable and $\partial_{i}$ the corresponding derivative with respect to the second variable. Then

$$
h \mapsto\left(A_{i} \partial_{i_{1}} \ldots \partial_{i_{n}} R_{\lambda}\right)(h ; g)
$$

is the kernel of the operator $A_{i} \partial_{i_{1}} \ldots \partial_{i_{n}}\left(\lambda I+H_{g}\right)^{-1}$ where the $\partial_{i}$ are now derivatives with respect to the explicit $g$-variable. This is clear from the formula

$$
\begin{aligned}
\left(\left(\lambda I+H_{k}\right)^{-1} \varphi\right)(g) & =\int_{G} d h R_{\lambda}(h ; k) \varphi\left(h^{-1} g\right) \\
& =\int_{G} d \hat{h} R_{\lambda}\left(g h^{-1} ; k\right) \varphi(h)
\end{aligned}
$$

But

$$
\partial_{i}\left(\lambda I+H_{g}\right)^{-1}=-\left(\lambda I+H_{g}\right)^{-1}\left(\partial_{i} H_{g}\right)\left(\lambda I+H_{g}\right)^{-1}
$$

as a consequence of the resolvent identity

$$
\left(\lambda I+H_{g_{1}}\right)^{-1}-\left(\lambda I+H_{g_{2}}\right)^{-1}=-\left(\lambda I+H_{g_{1}}\right)^{-1}\left(H_{g_{1}}-H_{g_{2}}\right)\left(\lambda I+H_{g_{2}}\right)^{-1} .
$$

Moreover

$$
\partial_{i} H_{g}=\sum_{\alpha ;|\alpha| \leq 2}\left(A_{i} c_{\alpha}\right)(g) A^{\alpha}
$$

Hence

$$
\partial_{i}\left(\lambda I+H_{g}\right)^{-1}=-\sum_{\alpha ;|\alpha| \leq 2}\left(A_{i} c_{\alpha}\right)(g)\left(\lambda I+H_{g}\right)^{-1} A^{\alpha}\left(\lambda I+H_{g}\right)^{-1} .
$$

Since $\left\|\partial_{i} c_{\alpha}\right\|_{\infty} \leq\left\|c_{\alpha}\right\|_{\infty ; 1}^{\prime}$ and $|\alpha| \leq 2$ we may use Lemma 4.4 to obtain the estimate

$$
\left\|\partial_{i}\left(\lambda I+H_{g}\right)^{-1}\right\|_{\infty \rightarrow \infty} \leq a_{1} \lambda^{-1}
$$

for all sufficiently large $\lambda$. The value of $a_{1}$ now of course depends on the norms $\left\|c_{\alpha}\right\|_{\infty ; 1}^{\prime}$. Furthermore

$$
\begin{gathered}
\partial_{i} \partial_{j}\left(\lambda I+H_{g}\right)^{-1}=-\sum_{\alpha ;|\alpha| \leq 2}\left(A_{i} A_{j} c_{\alpha}\right)(g)\left(\lambda I+H_{g}\right)^{-1} A^{\alpha}\left(\lambda I+H_{g}\right)^{-1} \\
+\sum_{\alpha ;|\alpha| \leq 2} \sum_{\beta ;|\beta| \leq 2}\left(A_{i} c_{\alpha}\right)(g)\left(A_{j} c_{\beta}\right)(g)\left\{\left(\lambda I+H_{g}\right)^{-1} A^{\alpha}\left(\lambda I+H_{g}\right)^{-1} A^{\beta}\left(\lambda I+H_{g}\right)^{-1}\right. \\
\left.\quad+\left(\lambda I+H_{g}\right)^{-1} A^{\beta}\left(\lambda I+H_{g}\right)^{-1} A^{\alpha}\left(\lambda I+H_{g}\right)^{-1}\right\}
\end{gathered}
$$


and again Lemma 4.4 gives bounds

$$
\left\|\partial_{i} \partial_{j}\left(\lambda I+H_{g}\right)^{-1}\right\|_{\infty \rightarrow \infty} \leq a_{2} \lambda^{-1}
$$

for large $\lambda$ with the value of $a_{2}$ dependent on the norms $\left\|c_{\alpha}\right\|_{\infty ; 2}$. Finally

$$
\begin{aligned}
A_{j} \partial_{i}\left(\lambda I+H_{g}\right)^{-1} & =\partial_{i} A_{j}\left(\lambda I+H_{g}\right)^{-1} \\
& =-\sum_{\alpha ;|\alpha| \leq 2}\left(A_{i} c_{\alpha}\right)(g) A_{j}\left(\lambda I+H_{g}\right)^{-1} A^{\alpha}\left(\lambda I+H_{g}\right)^{-1}
\end{aligned}
$$

and hence by Lemma 4.4

$$
\left\|A_{j} \partial_{i}\left(\lambda I+H_{g}\right)^{-1}\right\|_{\infty \rightarrow \infty} \leq a \lambda^{-1 / 2}
$$

with $a$ dependent on $\left\|c_{\alpha}\right\|_{\infty ; 1}^{\prime}$. By extension of this argument one then obtains the following.

Lemma 5.3 If the coefficients $c_{\alpha}$ of $H$ are in $L_{\infty ; n}^{\prime}$ then there exist $a_{n}, \lambda_{0}>0$ such that

$$
\begin{aligned}
\left\|\partial_{i_{1}} \ldots \partial_{i_{n}}\left(\lambda I+H_{g}\right)^{-1}\right\|_{\infty \rightarrow \infty} & \leq a_{n} \lambda^{-1} \\
\left\|A_{i} \partial_{i_{1}} \ldots \partial_{i_{n}}\left(\lambda I+H_{g}\right)^{-1}\right\|_{\infty \rightarrow \infty} & \leq a_{n} \lambda^{-1 / 2}
\end{aligned}
$$

for all $\lambda>\lambda_{0}, m \leq n$, and $1 \leq i, i_{k} \leq d^{\prime}$ where the values of $a_{n}$ and $\lambda_{0}$ depend on the coefficients of $H$ only through the ellipticity constant $\lambda_{c}$ and the norm

$$
\|c\|_{n}^{\prime}=\sum_{\alpha ;|\alpha| \leq 2}\left\|c_{\alpha}\right\|_{\infty ; n}^{\prime}
$$

Thus the estimates

$$
\begin{aligned}
& \underset{g \in G}{\operatorname{essup}} \int_{G} d h\left|\left(\partial_{i_{1}} \ldots \partial_{i_{n}} R_{\lambda}\right)(h ; g)\right| \leq a_{n} \lambda^{-1} \\
& \underset{g \in G}{\operatorname{ess} \sup _{G}} \int_{G} d h\left|\left(A_{i} \partial_{i_{1}} \ldots \partial_{i_{n}} R_{\lambda}\right)(h ; g)\right| \leq a_{n} \lambda^{-1 / 2}
\end{aligned}
$$

are valid under the same restrictions.

Moreover, if $c_{\alpha} \in L_{\infty ; n}$ then these bounds are valid with the $i_{k}$ in the range $1, \ldots, d$ but with $1 \leq i \leq d^{\prime}$. Then the values of $a_{n}$ and $\lambda_{0}$ also depend on the norm

$$
\|c\|_{n}=\sum_{\alpha ;|\alpha| \leq 2}\left\|c_{\alpha}\right\|_{\infty ; n}
$$


Now $R_{\lambda}$ is defined by (5.2) and we want to show that $R_{\lambda} C_{0}(G) \subseteq C_{0}(G)$. First if $\varphi$ has support in $\Omega$ and $\psi$ in $\Omega_{\psi}$ then $\left(R_{\lambda} \varphi\right)(g) \neq 0$ only for those $g$ such that there exists an $h \in \Omega_{\psi}$ with $h^{-1} g \in \Omega$, i.e., $R_{\lambda} \varphi$ is supported by $\Omega_{\psi} \Omega$, which is compact. But as $\left\|R_{\lambda}\right\|_{\infty \rightarrow \infty} \leq a \lambda^{-1}<\infty$ for large $\lambda$ it follows that $R_{\lambda}$ maps functions vanishing at infinity into functions vanishing at infinity.

Next we establish that $R_{\lambda}$ preserves continuity. But if $\varphi \in C_{c}(G)$ then

$$
\begin{aligned}
\left(R_{\lambda} \varphi\right)\left(g_{1}\right)-\left(R_{\lambda} \varphi\right)\left(g_{2}\right) & =\int_{G} d h \psi(h)\left(R_{\lambda}\left(h ; g_{1}\right)-R_{\lambda}\left(h ; g_{2}\right)\right) \varphi\left(h^{-1} g_{1}\right) \\
& +\int_{G} d h \psi(h) R_{\lambda}\left(h ; g_{2}\right)\left(\varphi\left(h^{-1} g_{1}\right)-\varphi\left(h^{-1} g_{2}\right)\right) .
\end{aligned}
$$

Moreover, for $c_{\alpha} \in L_{\infty ; 1}^{\prime}$ Lemma 5.3 gives bounds

$$
\sup _{g \in G} \int_{G} d h\left|\left(\partial_{i} R_{\lambda}\right)(h ; g)\right| \leq a \lambda^{-1}
$$

and

$$
\sup _{g \in G} \int_{G} d h\left|R_{\lambda}(h ; g)\right| \leq a \lambda^{-1}
$$

and as $\varphi$ is uniformly continuous on $G$ it follows that $\left(R_{\lambda} \varphi\right)\left(g_{1}\right) \rightarrow\left(R_{\lambda} \varphi\right)\left(g_{2}\right)$ as $g_{1} \rightarrow g_{2}$. Hence $R_{\lambda} \varphi$ is continuous. Finally by a density argument $R_{\lambda} C_{0} \subseteq C_{0}$.

Lemma 5.4 If $c_{\alpha} \in L_{\infty ; 1}^{\prime}$ for all $\alpha$ and $\lambda$ is large then $R_{\lambda} C_{0} \subseteq C_{0}$. Moreover, if $c_{\alpha} \in L_{\infty ; 2}^{\prime}$ then $R_{\lambda} C_{0 ; 1}^{\prime} \subseteq C_{0 ; 1}^{\prime}$ and one has bounds

$$
N_{\infty ; 1}^{\prime}\left(R_{\lambda} \varphi\right) \leq a \lambda^{-1 / 2}\|\varphi\|_{\infty}
$$

for all $\varphi \in C_{0}$ and all large $\lambda$ where the value of a depends on the ellipticity constant $\lambda_{c}$ and the norm $\|c\|_{1}^{\prime}$.

Proof The first statement has been proved above. But if $c_{\alpha} \in L_{\infty ; 2}^{\prime}$ and $\varphi \in C_{c}^{\infty}$, then $R_{\lambda} \varphi \in L_{\infty ; 1}^{\prime}$ and has compact support. Thus $R_{\lambda} C_{c}^{\infty} \subseteq C_{c}$. Moreover, (5.10) gives

$$
\begin{aligned}
\left(A_{i} R_{\lambda} \varphi\right)(g)= & \int_{G} d h \psi(h)\left(\left(\partial_{i} R_{\lambda}\right)(h ; g)+\left(A_{i} R_{\lambda}(h ; g)\right)\right) \varphi\left(h^{-1} g\right) \\
& +\int_{G} d h\left(A_{i} \psi\right)(h) R_{\lambda}(h ; g) \varphi\left(h^{-1} g\right)
\end{aligned}
$$

through integration by parts. Then estimating with (5.11), (5.12), and the bound

$$
\underset{g \in G}{\operatorname{ess} \sup _{G}} \int_{G} d h\left|\left(A_{i} R_{\lambda}\right)(h ; g)\right| \leq a \lambda^{-1 / 2}
$$


one deduces that

$$
\left\|A_{i} R_{\lambda} \varphi\right\|_{\infty} \leq a \lambda^{-1 / 2}\|\varphi\|_{\infty}
$$

Then $R_{\lambda} C_{0} \subseteq C_{0 ; 1}^{\prime}$ and (5.13) holds by continuity. The dependence of $a$ on the coefficients of $H$ follows from Lemma 5.3.

Next we estimate $Q_{\lambda}$. To this end we compute that

$$
\begin{aligned}
\left((\lambda I+H) R_{\lambda} \varphi\right)(g) & =\left(\lambda+\sum_{\alpha ;|\alpha| \leq 2} c_{\alpha}(g) A^{\alpha}\right)\left(\int_{G} d h \psi(h) R_{\lambda}(h ; g) \varphi\left(h^{-1} g\right)\right) \\
& =\left(\lambda+\sum_{\alpha ;|\alpha| \leq 2} c_{\alpha}(g) A^{\alpha}\right)\left(\int_{G} d \hat{h} \psi\left(g h^{-1}\right) R_{\lambda}\left(g h^{-1} ; g\right) \varphi(h)\right)
\end{aligned}
$$

Thus the differential operator $A^{\alpha}$, which acts on the $g$-variable, acts under the integral on $\psi$ and on both variables of the kernel $R_{\lambda}(\cdot ; \cdot)$. If we collect all terms for which $A^{\alpha}$ acts on the first variable of the kernel then their sum is

$$
\left(\lambda+\sum_{\alpha ;|\alpha| \leq 2} c_{\alpha}(g) A^{\alpha}\right) R_{\lambda}\left(g h^{-1} ; g\right)=\delta\left(g h^{-1}\right) \text {. }
$$

Here $A$ has the meaning of Lemma 5.3, a left derivative with respect to the first variable, and $\delta$ is the point measure in the $g$-variable, i.e.,

$$
\begin{aligned}
\int_{G} d g \psi(g)\left(\lambda+\sum_{\alpha ;|\alpha| \leq 2} c_{\alpha}(g) A^{\alpha}\right) R_{\lambda}\left(g h^{-1} ; g\right) & =\int_{G} d g \delta\left(g h^{-1}\right) \psi(g) \\
& =\Delta(h) \psi(h)
\end{aligned}
$$

for any $\psi \in C_{c}^{\infty}$. Hence, if the contribution to the expression $\left((\lambda I+H) R_{\lambda} \varphi\right)(g)$ of terms of this type is denoted by

$P_{\lambda}(g)$ then

$$
P_{\lambda}(g)=\int_{G} d \hat{h} \psi\left(g h^{-1}\right) \delta\left(g h^{-1}\right) \varphi(h)=\psi(e) \varphi(g)=\varphi(g) .
$$

Thus the remaining terms combine to give $\left(Q_{\lambda} \varphi\right)(g)$. In these terms at least one of the derivatives of each product $A^{\alpha}$ acts on $\psi$ or on the second variable of the kernel $R_{\lambda}(\cdot ; \cdot)$. Thus $\left(Q_{\lambda} \varphi\right)(g)$ is a finite linear combination of terms

$$
c(g) \int_{G} d h \chi(h) P_{\lambda}(h ; g) \varphi\left(h^{-1} g\right)
$$

where $c$ is one of the coefficients of $H, \chi$ is a derivative of $\psi$ of order less than or equal to two, and $P_{\lambda}(h ; g)$ is one of the following functions

$$
R_{\lambda}(h ; g), \quad\left(A_{i} R_{\lambda}\right)(h ; g), \quad\left(A_{i} \partial_{j} R_{\lambda}\right)(h ; g)
$$




$$
\left(\partial_{i} R_{\lambda}\right)(h ; g), \quad\left(\partial_{i} \partial_{j} R_{\lambda}\right)(h ; g)
$$

Thus since $c_{\alpha} \in L_{\infty ; 2}^{\prime}$ then

$$
\underset{g \in G}{\operatorname{esssup}}|c(g)| \int_{G} d h|\chi(h)| \cdot\left|P_{\lambda}(h ; g)\right| \leq a \lambda^{1 / 2}
$$

for all $\lambda>\lambda_{0}$ by Lemmas 5.1-5.3. Hence we have the first statement of the following lemma.

Lemma 5.5 If the coefficients $c_{\alpha}$ of $H$ are in $L_{\alpha ; 2}^{\prime}$ then there exist $a, \lambda_{0}>0$ such that

$$
\left\|Q_{\lambda}\right\|_{\infty \rightarrow \infty} \leq a \lambda^{-1 / 2}
$$

for all $\lambda>\lambda_{0}$ where the values of $a$, and $\lambda_{0}$, depend on the coefficients $c_{\alpha}$ only through $\lambda_{c}$ and the norm $\left\|c_{\alpha}\right\|_{2}^{\prime}$. Moreover, if $c_{\alpha} \in L_{\infty ; 3}^{\prime}$ then $Q_{\lambda} C_{0}(G) \subseteq C_{0}(G)$.

The proof of the second statement is the same as the corresponding proof for $R_{\lambda}$.

Additional smoothness properties of $R_{\lambda}$ and $Q_{\lambda}$ will be obtained in the course of subsequent arguments.

Remark In this section we have studied the properties of the right parametrix $R_{\lambda}$ of $H$. Similarly, one may define a left parametrix $R_{\lambda}^{L}$ of $H$ by

$$
\begin{aligned}
\left(R_{\lambda}^{L} \varphi\right)(g) & =\int_{G} d h \psi(h) R_{\lambda}^{\dagger}\left(h^{-1} ; h^{-1} g\right)(L(h) \varphi)(g) \\
& =\int_{G} d \hat{h} \psi\left(g h^{-1}\right) R_{\lambda}^{\dagger}\left(h g^{-1} ; h\right) \varphi(h)
\end{aligned}
$$

where $R_{\lambda}^{\dagger}(\cdot ; h)$ is now the fundamental solution of the operator $\lambda I+H_{h}^{\dagger}$, and

$$
\left(H_{h}^{\dagger} \varphi\right)(g)=\sum_{\alpha ;|\alpha| \leq 2} c_{\alpha}(h)\left((-A)^{\alpha_{*}} \varphi\right)(g)
$$

where $A^{\alpha_{*}}$ is equal to the product $A^{\alpha}$ but in the reverse order. In analogy with (5.3) one may now define a remainder term $Q_{\lambda}^{L}$ by

$$
R_{\lambda}^{L}(\lambda I+H)=I+Q_{\lambda}^{L}
$$

and prove that

$$
\left\|Q_{\lambda}^{L}\right\|_{\infty \rightarrow \infty}=O\left(\lambda^{-1 / 2}\right)
$$


which corresponds to (5.21). Note that if $R_{\lambda}$ is the right parametrix defined by (5.2), a simple computation shows that its adjoint is given by

$$
\left(R^{*} \psi\right)(g)=\int_{G} d \hat{h} \psi\left(h^{-1}\right) R_{\lambda}\left(h^{-1} ; h^{-1} g\right)(L(h) \varphi)(g)
$$

If we compare this with (5.22) we see that, except for first order terms, this left parametrix is the adjoint of the right parametrix corresponding to $\mathrm{H}^{\dagger}$, and this explains (and can be used as a basis for a proof of) (5.25). We will not make use of the left parametrix in the sequel. 


\section{Semigroups on $C_{0}$ and $L_{\infty}$}

Let $H$ denote the operator (1.17) defined on $C_{0 ; 2}^{\prime}$. We next argue that if $c_{\alpha} \in L_{\infty ; 3}^{\prime}$ for $|\alpha|=1,2, c_{0} \in C_{b}(G)$, and $H$ is subelliptic then it is norm-closable and its closure generates a continuous semigroup on $C_{0}$. First note that $c_{0}$ is a bounded multiplication operator on $C_{0}$ and hence it is a bounded perturbation of the corresponding $H$ with $c_{0}=0$. Hence we may assume $c_{0}=0$ in the generation proof. Secondly, remark that $H$, with $c_{0}=0$, is automatically dissipative on $C_{0 ; 2}^{\prime}$, i.e., if $\varphi \in C_{0 ; 2}^{\prime}$ and $\varphi\left(g_{0}\right)=\|\varphi\|_{\infty}$ then $(H \varphi)\left(g_{0}\right) \leq 0$. But dissipativity implies closability (see, for example, [Paz]). Thus to prove that the closure of $H$ generates a continuous contraction semigroup it suffices to prove that the range of $(\lambda I+H)$ is norm-dense for some large $\lambda$. Now suppose $\eta \in C_{0}^{*}$, the finite Baire measures on $G$, is orthogonal to the range of $(\lambda I+H)$. Then

$$
0=\left(\eta,(\lambda I+H) R_{\lambda} \varphi\right)=\left(\eta,\left(I+Q_{\lambda}\right) \varphi\right)
$$

for all $\varphi \in C_{c}^{\infty}$. But since $Q_{\lambda} C_{0} \subseteq C_{0}$ and $\left\|Q_{\lambda}\right\|_{\infty \rightarrow \infty} \leq a \lambda^{-1 / 2}$ for large $\lambda$, by Lemma 5.5 , this equation extends by continuity to all $\varphi \in C_{0}$. Now choosing $\lambda$ sufficiently large that $\left\|Q_{\lambda}\right\|_{\infty \rightarrow \infty}<1$ the operator $I+Q_{\lambda}$ is invertible and setting $\varphi=\left(I+Q_{\lambda}\right)^{-1} \psi$ one concludes that

$$
\left(\eta,(\lambda I+H) R_{\lambda} \varphi\right)=(\eta, \psi)=0
$$

for all $\psi \in C_{0}$. Therefore $\eta=0$, and the range of $(\lambda I+H)$ is dense for all large $\lambda$.

Although we have required that $c_{\alpha} \in L_{\infty ; 3}^{\prime}$ for $|\alpha|=1,2$ the condition on the first-order coefficients can easily be weakened.

Proposition 6.1 If $c_{\alpha} \in L_{\infty ; 3}^{\prime}$ for all $|\alpha|=2$ and $c_{\alpha} \in C_{b}$ for $|\alpha|<2$, then the subelliptic operator

$$
H=\sum_{\alpha ;|\alpha| \leq 2} c_{\alpha} A^{\alpha}
$$

on $C_{0}(G)$ is norm-closable and its closure generates a continuous semigroup. 
Proof If $H_{0}$ denotes the operator obtained from $H$ by setting $c_{\alpha}=0$ for $|\alpha|=0,1$ then its closure, which we also denote by $H_{0}$ generates a continuous semigroup. In particular $\left(\lambda I+H_{0}\right)^{-1}$ is a well defined bounded operator for all large $\lambda$. Next let $R_{\lambda}^{0}$ and $Q_{\lambda}^{0}$ denote the parametrix and remainder corresponding to $H_{0}$. Then

$$
\left(\lambda I+H_{0}\right) R_{\lambda}^{0} \varphi=\left(I+Q_{\lambda}^{0}\right) \varphi
$$

for all $\varphi \in C_{c}^{\infty}$ and hence

$$
\left(\lambda I+H_{0}\right)^{-1}\left(I+Q_{\lambda}^{0}\right) \varphi=R_{\lambda}^{0} \varphi
$$

for all large $\lambda$ and $\varphi \in C_{c}^{\infty}$. But this last identity extends to all $\varphi \in C_{0}$ by continuity. Consequently

$$
\left(\lambda I+H_{0}\right)^{-1} \varphi=R_{\lambda}^{0}\left(I+Q_{\lambda}^{0}\right)^{-1} \varphi
$$

for all $\varphi \in C_{0}$. But $R_{\lambda}^{0} C_{0 ; 1}^{\prime} \subseteq C_{0 ; 1}^{\prime}$ by Lemma 5.4 and

$$
\left\|A_{i}\left(\lambda I+H_{0}\right)^{-1}\right\|_{\infty \rightarrow \infty} \leq a \lambda^{-1 / 2}\left\|\left(I+Q_{\lambda}^{0}\right)^{-1}\right\|_{\infty \rightarrow \infty}
$$

by (5.11) and (6.1). Now choosing $\lambda_{0}$ such that $\left\|\left(I+Q_{\lambda}^{0}\right)^{-1}\right\|_{\infty \rightarrow \infty} \leq 2$ for $\lambda>\lambda_{0}$ one has bounds

$$
\left\|A_{i}\left(\lambda I+H_{0}\right)^{-1}\right\|_{\infty \rightarrow \infty} \leq 2 a \lambda^{-1 / 2}
$$

for $i=1, \ldots, d^{\prime}$ and $\lambda>\lambda_{0}$. These can be re-expressed in a perturbation theoretic form

$$
\left\|A_{i} \varphi\right\|_{\infty} \leq \varepsilon\left\|H_{0} \varphi\right\|_{\infty}+a^{\prime} \varepsilon^{-1}\|\varphi\|_{\infty}
$$

for all $\varphi \in C_{0 ; 2}^{\prime}$ and all $\varepsilon \in\langle 0,1]$. Then, since the first-order terms $H_{1}=\sum_{|\alpha|=1} c_{\alpha} A^{\alpha}$ in $H$ are dissipative, $H_{0}+H_{1}$ generates a continuous contraction semigroup by the perturbation theory of contraction semigroups. Finally since $c_{0}$ is bounded the closure of $H$ generates a continuous semigroup by the theory of bounded perturbations.

Note that the values of the constants $a$ and $a^{\prime}$ in (6.2) and (6.3) depend on the coefficients of $H_{0}$ through the norm $\|c\|_{2}^{\prime}$. This is a consequence of the estimates of Lemmas 5.4 and 5.5.

Proposition 6.1 has a direct analogue on $L_{\infty}$. 
Proposition 6.2 If $c_{\alpha} \in L_{\infty ; 3}^{\prime}$ for $|\alpha|=2$ and $c_{\alpha} \in L_{\infty}$ for $|\alpha|<2$ then the subelliptic operator

$$
H=\sum_{\alpha ;|\alpha| \leq 2} c_{\alpha} A^{\alpha}
$$

on $L_{\infty}(G ; d g)$ is weak*-weak $k^{*}$-closable and its closure generates a weakly* continuous semigroup.

Proof At this stage we only prove the proposition with the additional assumption $c_{\alpha} \in C_{b}$ for $|\alpha|=1$. The general case follows from the holomorphy of the corresponding semigroup, which will be established in Lemma 9.4, and the perturbation argument outlined below.

First set $c_{0}=0$. Then the corresponding closed $H$ generates a strongly continuous contraction semigroup on $C_{0}$ by Proposition 6.1. Moreover, it follows from (6.1) that

$$
(\lambda I+H)^{-1}=R_{\lambda}\left(I+Q_{\lambda}\right)^{-1}=\sum_{n \geq 0} R_{\lambda}\left(-Q_{\lambda}\right)^{n}
$$

for all large $\lambda$. Therefore the resolvent $(\lambda I+H)^{-1}$ extends by weak ${ }^{*}$-closure to a contraction on $L_{\infty}$, by Kaplansky's density theorem. .Thus $H$ is weak*-weak*-closed and generates a contraction semigroup on $L_{\infty}$. Finally $c_{0}$ is a bounded perturbation and hence the full $H$ is a generator.

The extension from $c_{\alpha} \in C_{b}$ to $c_{\alpha} \in L_{\infty}$ for the coefficients with $|\alpha|=1$ follows by a perturbation argument. If $H_{0}$ is the pure second-order part of $H$ then it follows from (6.2) and the foregoing extension argument that (6.3) is valid for all $\varphi \in L_{\infty ; 2}^{\prime}$. This in itself is not sufficient to make a perturbation argument because we are now dealing with weakly* continuous semigroups. But if in addition one has the holomorphy bound $\left\|H_{0} S_{t}^{0}\right\|_{\infty \rightarrow \infty} \leq a^{\prime \prime} t^{-1}$ for $t \in\langle 0,1]$ where $S^{0}$ is the semigroup generated by $H_{0}$ then (6.3) gives

$$
\left\|A_{i} S_{t}^{0}\right\|_{\infty \rightarrow \infty} \leq \varepsilon a^{\prime \prime} t^{-1}+\varepsilon^{-1} a^{\prime}
$$

for all $\varepsilon, t \in\langle 0,1]$. Hence setting $\varepsilon=t^{1 / 2}$ gives a bound $\left\|A_{i} S_{t}^{0}\right\|_{\infty \rightarrow \infty} \leq a t^{-1 / 2}$ which is integrable as $t \rightarrow 0$. Therefore one can apply 'time-dependent' perturbation theory to conclude that $H$ generates a weakly* continuous semigroup (see, for example, [BrR2] Theorem 3.1.33 or the proof of Proposition 11.1 below).

The holomorphy bounds which are necessary for the above argument will be established in Section 9. 
Despite this gap in the proof of Proposition 6.2 we have established-the following result for the subelliptic operator written in the symmetric form (1.20).

Corollary 6.3 If $c_{i j} \in L_{\infty ; 3}^{\prime}, c_{i} \in L_{\infty ; 1}^{\prime}$, and $c_{0} \in L_{\infty}$, then the subelliptic operator

$$
H=-\sum_{i, j=1}^{d^{\prime}} A_{i} c_{i j} A_{j}+\sum_{i=1}^{d^{\prime}}\left(c_{i} A_{i}+A_{i} c_{i}\right) / 2+c_{0}
$$

on $L_{\infty}$ is weak $k^{*}$-weak $k^{*}$-closable and its closure generates a weakly* continuous semigroup.

This follows by noting that $H$ can be written in the form (6.3) with $c_{\alpha} \in L_{\infty ; 3}^{\prime}$ if $|\alpha|=2, c_{\alpha} \in L_{\infty ; 1}^{\prime}$, if $|\alpha|=1$, and with zero-order term in $L_{\infty}$. But $L_{\infty ; 1}^{\prime} \subseteq C_{0}$ and hence the foregoing proof establishes the corollary.

Although the asymmetric form (6.4) of $H$ is most natural on $C_{0}$ and $L_{\infty}$ the symmetric form is more suited to the $L_{p}$-spaces with $p \in[1, \infty\rangle$ because duality arguments are important.

Next note that the inequalities (6.3) also follow for the operator $H$ with first order terms since

$$
H-H_{0}=\sum_{\alpha ;|\alpha|=0,1} c_{\alpha} A^{\alpha}
$$

and hence

$$
N_{\infty ; 1}^{\prime}(\varphi) \leq\left(\varepsilon\|H \varphi\|_{\infty}+a^{\prime} \varepsilon^{-1}\|\varphi\|_{\infty}\right)+\varepsilon\left(a_{1} N_{\infty ; 1}^{\prime}(\varphi)+a_{2}\|\varphi\|_{\infty}\right)
$$

But one can then solve these inequalities to obtain

$$
N_{\infty ; 1}^{\prime}(\varphi) \leq \varepsilon\|H \varphi\|_{\infty}+a \varepsilon^{-1}\|\varphi\|_{\infty}
$$

for all $\varphi \in C_{0 ; 2}^{\prime}$ and $\varepsilon \in\langle 0,1]$, where the value of the $a$ now depends on the ellipticity constant $\lambda_{c}$ and the norm $\|c\|_{2}^{\prime}$. This is a weak version of the $L_{\infty}$-conjecture of Section 1. The weakness is in the dependence of the parameter $a$ on the norm $\|c\|_{2}^{\prime}$ which precludes the extension of the inequalities (6.6) to operators with coefficients in $L_{\infty ; 1}^{\prime}$. Nevertheless, by approximation the inequalities extend to operators with leading coefficients in $L_{\infty ; 2}^{\prime}$.

If, however, the coefficients are in $L_{\infty ; 3}^{\prime}$ the inequalities extend to the closure of $H$ on $L_{\infty}$ and this another version of the basic $L_{\infty}$-conjecture. 
Proposition 6.4 If $H$ is a subelliptic operator of the form (1.17) and $c_{\alpha} \in L_{\infty ; 3}^{\prime}$ whenever $|\alpha|=2$ then there is an $a>0$ such that

$$
N_{\infty ; 1}^{\prime}(\varphi) \leq \varepsilon\|\bar{H} \varphi\|_{\infty}+a \varepsilon^{-1}\|\varphi\|_{\infty}
$$

for all $\varphi$ in the domain of the weak $k^{*}$-weak $k^{*}$-closure $\bar{H}$ of $H$ and all $\varepsilon \in\langle 0,1]$. The value of a depends on the coefficients of $H$ only through the parameters $\lambda_{c}$ and $\|c\|_{3}^{\prime}$.

Proof It follows from Corollary 6.3 that $H$ is weak*-weak*-closable and its closure $\bar{H}$ generates a weakly* continuous semigroup. But the semigroup generated by $\bar{H}$ leaves $C_{0}(G)$ globally invariant by Proposition 6.1 and the generator of the restricted semigroup is the norm closure of $H$, which we also denote by $H$. Since the estimate (6.7) is valid for $\varphi \in D(H)$, and since for each $\lambda>0$ the resolvent $(\lambda I+H)^{-1}$ is the restriction of $(\lambda I+\bar{H})^{-1}$ from $L_{\infty}$ to $C_{0}(G)$, one concludes that

$$
\begin{aligned}
\left\|A_{i}(\check{\lambda} I+H)^{-1} \varphi\right\|_{\infty} & \leq \varepsilon\left\|H(\lambda I+H)^{-1} \varphi\right\|_{\infty}+a \varepsilon^{-1}\left\|(\lambda I+H)^{-1} \varphi\right\|_{\infty} \\
& =\varepsilon\left\|\left(I-\lambda(\lambda I+H)^{-1}\right) \varphi\right\|_{\infty}+a \varepsilon^{-1}\left\|(\lambda I+H)^{-1} \varphi\right\|_{\infty} \\
& \leq 2 \varepsilon\|\varphi\|_{\infty}+a \lambda^{-1} \varepsilon^{-1}\|\varphi\|_{\infty} .
\end{aligned}
$$

Moreover, as $(\lambda I+\bar{H})^{-1}$ is weakly* continuous and $A_{i}$ is weak*-weak*-closed, and the unit sphere in $L_{\infty}$ is weak*-compact, it follows from Kaplansky's density theorem that the last inequality extends to all $\varphi \in L_{\infty}$. Therefore setting $\varepsilon=\lambda^{-1 / 2}$ we obtain

$$
\left\|A_{i}(\lambda I+\bar{H})^{-1} \varphi\right\|_{\infty} \leq a^{\prime} \lambda^{-1 / 2}\|\varphi\|_{\infty}
$$

for all $\varphi \in L_{\infty}$. Replacing $\varphi$ by $(\lambda I+\bar{H}) \varphi$ one then deduces that

$$
\left\|A_{i} \varphi\right\|_{\infty} \leq a^{\prime} \lambda^{-1 / 2}\|\bar{H} \varphi\|_{\infty}+a^{\prime} \lambda^{1 / 2}\|\varphi\|_{\infty} .
$$

Finally, setting $\varepsilon=a^{\prime} \lambda^{-1 / 2}$ one obtains (6.7) for all $\varphi \in D(\bar{H})$.

Note that the arguments used to prove the proposition also allow one to extend the conclusions of Proposition 3.2.

Corollary 6.5 If $H$ is a subelliptic operator of the form (1.17) and $c_{\alpha} \in L_{\infty ; 3}^{\prime}$ whenever $|\alpha|=2$ then for each $\delta>0$ there is an $a_{\delta}>0$ such that

$$
N_{\infty ; 1}^{\prime}(\varphi) \leq \varepsilon\|\bar{H} \varphi\|_{\infty}+a_{\delta} \varepsilon^{-1-\delta}\|\varphi\|_{\infty}
$$

for all $\varphi$ in the domain of the weak $k^{*}$-weak $k^{*}$-closure $\bar{H}$ of $H$ and all $\varepsilon \in\langle 0,1]$. The value of $a_{\delta}$ depends on the coefficients of $H$ only through the ellipticity constant $\lambda_{c}$ and the norm $\|c\|_{1}^{\prime}$. 
We return to further discussion of a priori inequalities in the next two sections and again in Section 10. 


\section{Semigroups on $L_{1}$}

Let $H$ denote a subelliptic operator of the form (1.17) on $L_{1 ; 2}^{\prime}$. If $c_{\alpha} \in L_{\infty ;|\alpha|}^{\prime}$ then the adjoint $H^{*}$ of $H$ is defined on $L_{\infty ; 2}^{\prime}$. The restriction of $H^{*}$ to $L_{\infty ; 2}^{\prime}$, which we call the formal adjoint $H^{\dagger}$, is a subelliptic operator of the same form.

$$
H^{\dagger}=\sum_{\alpha ;|\alpha| \leq 2} c_{\alpha}^{\dagger} A^{\alpha}
$$

The coefficients $c_{\alpha}^{\dagger}$ are uniquely determined by the $c_{\alpha}$ and since $c_{\alpha}^{\dagger}=c_{\alpha}$ for $|\alpha|=2$ the ellipticity constants coincide. Similarly if $H$ is defined on $L_{\infty ; 2}^{\prime}$ one can define its formal adjoint on $L_{1 ; 2}^{\prime}$ and then $H^{\dagger \dagger}=H$.

Next suppose $H$ acts on $L_{1 ; 2}^{\prime}, c_{\alpha} \in L_{\infty ; 3}^{\prime}$ if $|\alpha|=2$, and $c_{\alpha} \in L_{\infty ; 1}^{\prime}$ if $|\alpha|=1$. Then $H^{\dagger}$ on $L_{\infty ; 2}^{\prime}$ has coefficients $c_{\alpha}^{\dagger}=c_{\alpha} \in L_{\infty ; 3}^{\prime}$ if $|\alpha|=2$, and $c_{\alpha}^{\dagger} \in L_{\infty ; 1}^{\prime}$ if $|\alpha|=1$. Therefore the $L_{\infty}$-closure of $H^{\dagger}$ generates a weakly* continuous semigroup on $L_{\infty}$ by Proposition 6.2. Thus the adjoint $H^{\dagger *}$ of $H^{\dagger}$ is the generator of a strongly continuous semigroup on the predual $L_{1}$. (If $H^{\dagger}$ generates $S^{\dagger}$ on $L_{\infty}$ then $H^{\dagger *}$ generates $S=S^{\dagger *}$ on $L_{1}$.) But $H^{\dagger *}$ is an extension of $H^{\dagger \dagger}=H$. Thus if we can establish that the $L_{1}$ closure $\bar{H}$ of $H$ satisfies $\bar{H}=H^{\dagger *}$ then $\bar{H}$ generates the strongly continuous semigroups $S$ on $L_{1}$. To this end it suffices to show that the range $R(\lambda I+H)$ of $(\lambda I+H)$ is dense in $L_{1}$ for some large positive $\lambda$.

The same tactic also applies to $H$ on $L_{\hat{1} ; 2}^{\prime}$ but the change of measure alters the duality arguments. Relative to the inner product

$$
\begin{aligned}
\langle\varphi, \psi\rangle & =\int_{G} d \hat{g} \bar{\varphi}(g) \psi(g) \\
& =\int_{G} d g \Delta(g)^{-1} \bar{\varphi}(g) \psi(g)=\left(\Delta^{-1} \varphi, \psi\right)
\end{aligned}
$$

one has the relation

$$
\begin{aligned}
\langle H \varphi, \psi\rangle & =\left(\Delta^{-1} H \varphi, \psi\right) \\
& =\left(\varphi, H^{\dagger} \Delta^{-1} \psi\right)=\left\langle\varphi, \delta\left(H^{\dagger}\right) \psi\right\rangle
\end{aligned}
$$

for $\varphi \in L_{\hat{p} ; 2}^{\prime}$ and $\psi \in L_{\hat{q} ; 2}^{\prime}$, where $p$ and $q$ are conjugate and $\delta\left(H^{\dagger}\right)=\Delta H^{\dagger} \Delta^{-1}$. This operator is, however, a subelliptic operator of the type under consideration. It 
is obtained from $H^{\dagger}$ by replacing $A_{i}$ by $A_{i}-\beta_{i} I$ where $\beta_{i}=\left(A_{i} \Delta\right)(e)$. Thus to decide that the $L_{\hat{1}}$-closure $\bar{H}$ of $H$ generates a strongly continuous semigroup $\hat{S}$ on $L_{\hat{1}}$ it suffices to show that $\bar{H}=\delta\left(H^{\dagger}\right)^{*}$, where the star now denotes the adjoint with respect to the inner product $\langle\cdot, \cdot\rangle$. This is achieved by a simple modification of the subsequent $L_{1}$-proof.

Let $R_{\lambda}^{\dagger}$ and $Q_{\lambda}^{\dagger}$ denote the parametrix and remainder associated with $H^{\dagger}$ on $L_{\infty}$. Then

$$
\left(\lambda I+H^{\dagger}\right)^{-1}=R_{\lambda}^{\dagger}\left(I+Q_{\lambda}^{\dagger}\right)^{-1}
$$

where we have again simplified notation by using $H^{\dagger}$ to denote the closed operator. Hence

$$
\left(\lambda I+H^{\dagger *}\right)^{-1}=\left(I+Q_{\lambda}^{\dagger *}\right)^{-1} R_{\lambda}^{\dagger *}
$$

on $L_{1}$, or

$$
\left(\lambda I+H^{\dagger *}\right)\left(I+Q_{\lambda}^{\dagger *}\right)^{-1} R_{\lambda}^{\dagger *} \psi=\psi
$$

for all $\psi \in L_{1}$. But if $\psi \in L_{1}$ has the property that

$$
\left(I+Q_{\lambda}^{\dagger *}\right)^{-1} R_{\lambda}^{\dagger *} \psi \in L_{1 ; 2}^{\prime}=D(H)
$$

then

$$
(\lambda I+H)\left(I+Q_{\lambda}^{\dagger *}\right)^{-1} R_{\lambda}^{* *} \psi=\psi
$$

Now we argue below that $\left(I+Q_{\lambda}^{\dagger *}\right)^{-1} R_{\lambda}^{\dagger *}$ is bounded as an operator from $L_{1 ; 2}^{\prime}$ into $L_{1 ; 2}^{\prime}$ if the second-order coefficients are sufficiently smooth. Hence it follows that $R(\lambda I+H) \supseteq L_{1 ; 2}^{\prime}$ and since $L_{1 ; 2}^{\prime}$ is $L_{1}$-dense in $L_{1}$ one concludes that $\bar{H}=H^{\dagger *}$.

Lemma 7.1 If $c_{\alpha}^{\dagger} \in L_{\infty ; n+2}$ for all $\alpha$ then $\left(I+Q_{\lambda}^{\dagger *}\right)^{-1} R_{\lambda}^{\dagger *}$ is bounded as an operator from $L_{1 ; n}$ into $L_{1 ; n}$ for all sufficiently large $\lambda$.

Proof In Section 5 we established that $R_{\lambda}^{\dagger}$ and $Q_{\lambda}^{\dagger}$ are operators of the form

$$
\begin{aligned}
& \left(R_{\lambda}^{\dagger} \varphi\right)(g)=\int_{G} d h R(h ; g) \varphi\left(h^{-1} g\right) \\
& \left(Q_{\lambda}^{\dagger} \varphi\right)(g)=\int_{G} d h Q(h ; g) \varphi\left(h^{-1} g\right)
\end{aligned}
$$


where, by Łemma 5.3, the kernels $R, Q$ satisfy estimates

$$
\begin{aligned}
& \underset{g \in G}{\operatorname{ess} \sup _{G}} \int_{G} d h\left|\left(\partial_{i_{1}} \ldots \partial_{i_{m}} R\right)(h ; g)\right| \leq a_{n} \lambda^{-1} \\
& \underset{g \in G}{\operatorname{essup}} \int_{G} d h\left|\left(\partial_{i_{1}} \ldots \partial_{i_{m}} Q\right)(h ; g)\right| \leq a_{n} \lambda^{-1 / 2}
\end{aligned}
$$

for all large $\lambda$ and $m \leq n+2$ where $1 \leq i_{k} \leq d$. Also $R$ and $Q$ have compact support around $e$ in the first variable. Then a simple computation establishes that

$$
\left(R_{\lambda}^{\dagger *} \varphi\right)(g)=\int_{G} d h R(h ; h g) \varphi(h g)
$$

with a similar action for $Q_{\lambda}^{\dagger *}$. Thus the $n$-th term in the expansion

$$
\left(I+Q_{\lambda}^{\dagger *}\right)^{-1} R_{\lambda}^{\dagger *} \varphi=\sum_{n \geq 0}\left(-Q_{\lambda}^{\dagger *}\right)^{n} R_{\lambda}^{\dagger *} \varphi
$$

has the form

$$
\begin{aligned}
&\left(\left(Q_{\lambda}^{\dagger *}\right)^{n} R_{\lambda}^{\dagger *} \varphi\right)(g)= \int_{G} d h_{0} \ldots \int_{G} d h_{n} Q\left(h_{n} ; h_{n} g\right) \ldots \\
& \ldots Q\left(h_{1} ; h_{1} h_{2} \ldots h_{n} g\right) R\left(h_{0} ; h_{0} h_{1} \ldots h_{n} g\right) \\
& \cdot \varphi\left(h_{0} h_{1} \ldots h_{n} g\right) .
\end{aligned}
$$

Applying $A_{i}$ to this expression amounts to taking the left derivative with respect to $g$ in the direction $a_{i}$. Thus we have to consider expressions such as

$$
\begin{aligned}
\left(A_{i} Q\right)(h ; k g) & =\left.\frac{d}{d t} Q\left(h ; k e^{-t a_{i}} g\right)\right|_{t=0} \\
& =\left.\frac{d}{d t} Q\left(h ; e^{-t k a_{i} k^{-1}} k g\right)\right|_{t=0} \\
& =\left(d L\left(k a_{i} k^{-1}\right) Q\right)(h ; k g)
\end{aligned}
$$

where the left derivative is with respect to the second variable of $Q$. But

$$
d L\left(k a_{i} k^{-1}\right)=\sum_{j=1}^{d} b_{i j}(k) \partial_{j}
$$

where the coefficients $b_{i j} \in C^{\infty}, b_{i j}(e)=\delta_{i j}$, and the $b_{i j}$ together with their derivatives are at most exponentially increasing. Hence we obtain an estimate

$$
\left|\left(A_{i} Q\right)(h ; k g)\right| \leq b e^{\omega|k|} \sum_{j=1}^{d}\left|\left(\partial_{j} Q\right)(h ; k g)\right| .
$$


Similarly -

$$
\left|\frac{d}{d t} \varphi\left(k e^{-t a_{i}} g\right)\right|_{t=0} \leq b e^{\omega|k|} \sum_{j=1}^{d}\left|\left(A_{j} \varphi\right)(k g)\right|
$$

Therefore we obtain an estimate

$$
\begin{gathered}
\left|\left(A_{i}\left(Q_{\lambda}^{* *}\right)^{n} R_{\lambda}^{\dagger *} \varphi\right)(g)\right| \leq \quad b \sum_{k=1}^{n} \sum_{j=1}^{d} \int_{G} d h_{0} \ldots \int_{G} d h_{n}\left|Q\left(h_{n} ; h_{n} g\right)\right| \ldots \\
\quad \ldots e^{\omega\left|h_{k} \ldots h_{n}\right|}\left|\left(\partial_{j} Q\right)\left(h_{k} ; h_{k} \ldots h_{n} g\right)\right| \ldots\left|Q\left(h_{1} ; h_{1} \ldots h_{n} g\right)\right| \cdot \\
\cdot\left|R\left(h_{0} ; h_{0} \ldots h_{n} g\right)\right| \cdot\left|\varphi\left(h_{0} \ldots h_{n} g\right)\right| \\
+b \sum_{j=1}^{d} \int_{G} d h_{0} \ldots \int_{G} d h_{n}\left|Q\left(h_{n} ; h_{n} g\right)\right| \ldots\left|Q\left(h_{1} ; h_{1} \ldots h_{n} g\right)\right| \\
\quad \cdot e^{\omega\left|h_{0} \ldots h_{n}\right|}\left\{\left|\left(\partial_{j} R\right)\left(h_{0} ; h_{0} \ldots h_{n} g\right)\right| \cdot\left|\varphi\left(h_{0} \ldots h_{n} g\right)\right|\right. \\
+ \\
\left.+R\left(h_{0} ; h_{0} \ldots h_{n} g\right)|\cdot|\left(\partial_{j} \varphi\right)\left(h_{0} \ldots h_{n} g\right) \mid\right\}
\end{gathered}
$$

Now the kernels $Q, R$ have support in the first variable in a ball $B_{r}=\{h \in G ;|h| \leq$ $r\}$. Consequently

$$
e^{\omega\left|h_{k} \ldots h_{n}\right|} \leq e^{\omega(n-k+1) r} \leq e^{\omega(n+1) r}
$$

in the region where the integrand is non-zero. Next we use the observation that the $L_{1} \rightarrow L_{1}$ norm of an operator of the type

$$
(P \varphi)(g)=\int_{G} d h P(h ; h g) \varphi(h g)
$$

is equal to the $L_{\infty} \rightarrow L_{\infty}$ norm of the adjoint operator

$$
\left(P^{*} \varphi\right)(g)=\int_{G} d h P(h ; g) \varphi\left(h^{-1} g\right)
$$

and the latter norm is given by

$$
\left\|P^{*}\right\|_{\infty \rightarrow \infty}=\underset{g \in G}{\operatorname{essup}} \int_{G} d h|P(h ; g)| .
$$

Hence we obtain from Lemmas 5.3 and 5.5 the bounds

$$
\left\|A_{i}\left(Q_{\lambda}^{\dagger *}\right)^{n} R_{\lambda}^{\dagger *} \varphi\right\|_{1} \leq b d\left(a e^{\omega r} \lambda^{-1 / 2}\right)^{n+1}(n+2)\|\varphi\|_{1 ; 1}
$$

for all large $\lambda$. One then concludes that if $\lambda$ is sufficiently large, i.e., if $a e^{\omega r} \lambda^{-1 / 2}<1$, then $\left(I+Q_{\lambda}^{\dagger *}\right)^{-1} R_{\lambda}^{\dagger *}$ is a bounded operator from $L_{1 ; 1}$ to $L_{1 ; 1}$. 
At thispoint we have established Lemma 7.1 for $n=1$. But for higher $n$ the proof follows by successive differentiation and repetition of the above estimation procedure using Lemmas 5.3 and 5.5.

If one now considers the operator (7.1) with $c_{\alpha}^{\dagger} \in L_{\infty ; 4}$ for all $\alpha$ then Lemma 7.1 implies that the closure of $H=H^{\dagger \dagger}$ on $L_{1 ; 2}^{\prime}$ generates a strongly continuous semigroup on $L_{1}$.

Proposition 7.2 If $c_{\alpha} \in L_{\infty ; 5}$ for $|\alpha|=2$ and $c_{\alpha} \in L_{\infty ; 4}$ for $|\alpha|=1$ then the $L_{1}$-closure of $H$ generates a strongly continuous semigroup $S$ on $L_{1}$. Moreover, the $L_{\hat{1}}$-closure of $H$ generates a strongly continuous semigroup $\hat{S}$ on $L_{\hat{1}}$.

Proof It follows from the adjoint relation that $c_{\alpha}^{\dagger} \in L_{\infty ; 5}$ for $|\alpha|=2$ and $c_{\alpha}^{\dagger} \in L_{\infty ; 4}$ for $|\alpha|=1$. Now $H_{0}^{\dagger}=H^{\dagger}-c_{0}$ is the formal adjoint of a subelliptic operator $H_{0}$ on $L_{1}$ which is a bounded perturbation of $H$. Applying Lemma 7.1 to $H_{0}^{\dagger}$ one deduces that $\left(I+Q_{\lambda}^{\dagger *}\right)^{-1} R_{\lambda}^{\dagger *}$ is bounded as an operator from $L_{1 ; 2}$ into $L_{1 ; 2}$ for all large $\lambda$. Therefore by (7.4) the range of $\left(\lambda I+H_{0}\right)$ contains the dense subset $L_{1 ; 2}$ of $L_{1}$. Consequently $\bar{H}_{0}=H_{0}^{\dagger *}$ and $\bar{H}_{0}$ is the generator of a strongly continuous semigroup on $L_{1}$. Finally $\bar{H}$ is a generator because it is a bounded perturbation of $\bar{H}_{0}$.

The proof that the $L_{\hat{1}}$-closure of $H$ is a generator follows by a similar argument but with $H^{\dagger}$ on $L_{\infty}$ replaced by $\delta\left(H^{\dagger}\right)=\Delta H^{\dagger} \Delta^{-1}$ and the inner product $(\cdot, \cdot)$ replaced by the inner product (7.2).

A similar result holds for $H$ written in the symmetric form (1.20).

Corollary 7.3 If $c_{i j} \in L_{\infty ; 5}, c_{i} \in L_{\infty ; 4}$, and $c_{0} \in L_{\infty}$, then the subelliptic operator

$$
H=-\sum_{i, j=1}^{d^{\prime}} A_{i} c_{i j} A_{j}+\sum_{i=1}^{d^{\prime}}\left(c_{i} A_{i}+A_{i} c_{i}\right) / 2+c_{0}
$$

on $L_{1}$ is norm-closable and its closure generates a strongly continuous semigroup $S$. Moreover, the operator is closable on $L_{\hat{1}}$ and its closure generates a second continuous semigroup $\hat{S}$.

Proof The condition on the coefficients ensures that the coefficients $c_{\alpha}^{\dagger},|\alpha|=1,2$ of the formal adjoint $H^{\dagger}$ on $L_{\infty}$, of $H$ on $L_{1}$, are in $L_{\infty ; 4}$. Therefore the result follows from Proposition 7.2. 
At this stage we have constructed semigroups generated by the closure of $H$ on $L_{\infty}, L_{1}$ and $L_{\hat{1}}$ but we have not considered their interpolation properties nor the consistency of their action on $L_{1}$ and $L_{\hat{1}}$. The only connection at this point between these semigroups is by duality. The semigroup on $L_{1}$ is the dual relative to the left invariant Haar measure of the semigroup generated by the formal adjoint $H^{\dagger}$ on $L_{\infty}$. The semigroup on $L_{\hat{1}}$ is the dual relative to the right invariant Haar measure of the semigroup generated by the formal adjoint $\delta\left(H^{\dagger}\right)$ on $L_{\infty}$. 


\section{Semigroups on $L_{p}, p \in\langle 1, \infty\rangle$}

Up to this point we have concentrated on subelliptic operators of the form (1.17) because we have principally used $L_{\infty}$-techniques. In the next five sections, however, duality arguments and $L_{2}$-estimates play an important role. Hence it is more appropriate to consider $H$ in the symmetric form (1.20). If $c_{i j} \in L_{\infty ; 5}, c_{i} \in L_{\infty ; 4}$, and $c_{0} \in L_{\infty}$ then the closure $H_{\infty}$ of $H$ on $L_{\infty}$ generates a weakly* continuous semigroup $S^{(\infty)}$ on $L_{\infty}$ by Corollary 6.3. Moreover, since $H_{\infty}-\omega I$ with $\omega=\left\|c_{0}\right\|_{\infty}+\sum_{i=1}^{d^{\prime}}\left\|A_{i} c_{i}\right\|_{\infty} / 2$ is dissipative one has bounds

$$
\left\|S_{t}^{(\infty)}\right\|_{\infty \rightarrow \infty} \leq e^{\omega t}
$$

In addition the closure $H_{1}$ of $H$ on $L_{1}$ generates a strongly continuous semigroup $S^{(1)}$ on $L_{1}$ by Proposition 7.3 and by duality one has bounds

$$
\left\|S_{t}^{(1)}\right\|_{1 \rightarrow 1} \leq e^{\omega t}
$$

Next we argue that these semigroups coincide on $L_{1} \cap L_{\infty}$ and then we can apply the Riesz-Thorin interpolation theorem and obtain an interpolating semigroup on the $L_{p}$-spaces. Subsequently we examine the corresponding semigroups on the $L_{\hat{p}^{-} \text {-spaces. }}$

In order to deduce that the semigroups coincide on $L_{1} \cap L_{\infty}$ it suffices to prove that the resolvents $\left(\lambda I+H_{1}\right)^{-1}$ and $\left(\lambda I+H_{\infty}\right)^{-1}$ coincide on $L_{1} \cap L_{\infty}$ for all large $\lambda$. To this end, define

$$
\mathcal{L}=R_{\lambda}\left(I+Q_{\lambda}\right)^{-1} C_{c}^{2}
$$

where the $R_{\lambda}$ are viewed as bounded operators on $L_{\infty} \supseteq C_{c}^{2}$. We will establish that

$$
\mathcal{L} \subseteq L_{1,2} \cap L_{\infty ; 2} \subseteq D\left(H_{1}\right) \cap D\left(H_{\infty}\right)
$$

Since

$$
(\lambda I+H) \mathcal{L}=C_{c}^{2}
$$

and $C_{c}^{2}$ is dense both in $L_{1}$ and $L_{\infty}$ it then follows that $\mathcal{L}$ is a core of both $H_{1}$ and $H_{\infty}$. Furthermore

$$
\left.\left(\lambda I+H_{1}\right)^{-1}\right|_{C_{c}^{2}}=\left.R_{\lambda}\left(I+Q_{\lambda}\right)^{-1}\right|_{C_{c}^{2}}=\left.\left(\lambda I+H_{\infty}\right)^{-1}\right|_{C_{c}^{2}} .
$$


Therefore the semigroups must coincide on $L_{1} \cap L_{\infty}$. Thus it remains- to prove the following.

Lemma 8.1 If $c_{i j} \in L_{\infty ; 5}, c_{i} \in L_{\infty ; 4}$ and $c_{0} \in L_{\infty}$ then

$$
\mathcal{L} \subseteq L_{1 ; 2} \cap L_{\infty ; 2}
$$

Proof The proof is a variation of the argument used to establish Lemma 7.1. Setting

$$
\begin{aligned}
& \left(R_{\lambda} \varphi\right)(g)=\int_{G} d h R(h ; g) \varphi\left(h^{-1} g\right) \\
& \left(Q_{\lambda} \varphi\right)(g)=\int_{G} d h Q(h ; g) \varphi\left(h^{-1} g\right)
\end{aligned}
$$

the kernels $R$ and $Q$ satisfy estimates

$$
\begin{aligned}
& \underset{g \in G}{\operatorname{esssup}} \int_{G} d h\left|\left(\partial_{i_{1}} \ldots \partial_{i_{m}} R\right)(h ; g)\right| \leq a \lambda^{-1} \\
& \underset{g \in G}{\operatorname{esssup}} \int_{G} d h\left|\left(\partial_{i_{1}} \ldots \partial_{i_{m}} Q\right)(h ; g)\right| \leq a \lambda^{-1}
\end{aligned}
$$

for $\lambda>\lambda_{0}, m=1,2$, and $i_{k}=1, \ldots, d$, by Lemma 5.3 .

Moreover, both kernels have compact support in the first variable. Now using

$$
\left(R_{\lambda}\left(I+Q_{\lambda}\right)^{-1} \varphi\right)(g)=\sum_{n \geq 0}\left(R_{\lambda}\left(-Q_{\lambda}\right)^{n} \varphi\right)(g)
$$

one argues as in the proof of Lemma 7.1 that this sum, together with its derivatives of order one or two in the $g$-variable, converges in $L_{\infty}$ for all large $\lambda$. This establishes that $\mathcal{L} \subseteq L_{\infty ; 2}$. One also obtains estimates

$$
\left\|A^{\alpha} R_{\lambda} Q_{\lambda}^{n} \varphi\right\|_{\infty} \leq a n^{|\alpha|}\left(b \lambda^{-1 / 2}\right)^{n+1}\|\varphi\|_{\infty ;|\alpha|}
$$

for suitable $a, b>0$ and all $\alpha$ with $|\alpha| \leq 2$.

Now to obtain the $L_{1}$-estimates assume $\varphi$ has support in the ball $B_{r_{0}}=\{g \in$ $\left.G ;|g| \leq r_{0}\right\}$ and that the support of the kernels $R, Q$ with respect to the first variable is contained in $B_{r}$. Then the support of $A^{\alpha} R_{\lambda} Q_{\lambda}^{n} \varphi$ is in $B_{r_{0}+(n+1) r}$. But the volume of $B_{r}$ grows at most exponentially. Hence one has bounds

$$
\left|B_{r_{0}+(n+1) r}\right| \leq k e^{\omega\left(r_{0}+(n+1) r\right)}
$$

for all $n=0,1, \ldots$ Thus

$$
\left\|A^{\alpha} R_{\lambda} Q_{\lambda}^{n} \varphi\right\|_{1} \leq\left\|A^{\alpha} R_{\lambda} Q_{\lambda}^{n} \varphi\right\|_{\infty}\left|B_{r_{0}+(n+1) r}\right| \leq a^{\prime} n^{|\alpha|}\left(b^{\prime} \lambda^{-1 / 2}\right)^{n+1}\|\varphi\|_{\infty ;|\alpha|}
$$


for suitable $a^{\prime}, b^{\prime}>0$ and all $\alpha$ with $|\alpha| \leq 2$ by (8.2) and (8.3). Therefore if $\lambda$ is sufficiently large then $A^{\alpha} R_{\lambda}\left(I+Q_{\lambda}\right)^{-1} \varphi \in L_{1}$ for $|\alpha| \leq 2$. This establishes that $\mathcal{L} \subseteq L_{1 ; 2}$ and completes the proof of the lemma.

At this point we can establish the existence of an interpolating semigroup on the $L_{p}$-spaces.

Proposition 8.2 If $c_{i j} \in L_{\infty ; 5}, c_{i} \in L_{\infty ; 4}$, and $c_{0} \in L_{\infty}$ then the $L_{p}$-closure of the corresponding subelliptic operator $H$ on $L_{p ; 2}$ generates a continuous semigroup $S^{(p)}$ and

$$
S_{t}^{(p)} \varphi=S_{t}^{(r)} \varphi
$$

for all $\varphi \in L_{p}^{-} \cap L_{r}$ and $p, r \in[1, \infty]$. Moreover, the $L_{\hat{p}}$-closure of $H$ on $L_{\hat{p} ; 2}$ generates a continuous. semigroup $\hat{S}^{(p)}$ and

$$
\hat{S}_{t}^{(p)} \varphi=\hat{S}_{t}^{(r)} \varphi
$$

for all $\varphi \in L_{\hat{p}} \cap L_{\hat{r}}$ and $p, r \in[1, \infty]$. The semigroups $S^{(p)}$ and $\hat{S}^{(p)}$ are consistent, i.e., $S_{t}^{(p)}\left(L_{p} \cap L_{\hat{p}}\right) \subseteq L_{p} \cap L_{\hat{p}}, \hat{S}_{t}^{(p)}\left(L_{p} \cap L_{\hat{p}}\right) \subseteq L_{p} \cap L_{\hat{p}}$ and

$$
S_{t}^{(p)} \varphi=\hat{S}_{t}^{(p)} \varphi
$$

for all $\varphi \in L_{p} \cap L_{\hat{p}}$ and $p \in[1, \infty]$.

Proof We have constructed $S^{(1)}$ in Proposition 7.2 and $S^{(\infty)}$ in Corollary 6.3 and proved they coincide on $L_{1} \cap L_{\infty}$ as a consequence of Lemma 8.1. Thus the RieszThorin theorem gives a family $S^{(p)}$ of semigroups on the $L_{p}$-spaces which coincide with $S^{(1)}$, and $S^{(\infty)}$, on $L_{1} \cap L_{p}$, and $L_{p} \cap L_{\infty}$, respectively. To prove that $S^{(p)}$ is continuous let $\varphi \in L_{1 ; 2} \cap L_{\infty ; 2}$. Then $t \mapsto S_{t}^{(p)} \varphi$ is differentiable at $t=0$ both in $L_{1}$ and in $L_{\infty}$. Moreover

$$
t^{-1}\left\|\left(I-S_{t}^{(p)}\right) \varphi\right\|_{p} \leq\left(t^{-1}\left\|\left(I-S_{t}^{(1)}\right) \varphi\right\|_{1}\right)^{1-1 / p}\left(t^{-1}\left\|\left(I-S_{t}^{(\infty)}\right) \varphi\right\|_{\infty}\right)^{1 / p}
$$

and it follows that

$$
\sup _{0<t \leq 1} t^{-1}\left\|\left(I-S_{t}^{(p)}\right) \varphi\right\|_{p}<\infty .
$$

Since $L_{p}$ is reflexive for $p \in\langle 1, \infty\rangle$ this implies that $t \mapsto S_{t}^{(p)} \varphi$ is differentiable at $t=0$ and as $L_{1 ; 2} \cap L_{\infty ; 2}$ is $L_{p}$-dense $S^{(p)}$ must be strongly continuous. Moreover, the 
bound implies that $\varphi \in D\left(H_{p}\right)$, where $H_{p}$ is the generator of $S^{(p)}$, and $H_{p} \varphi=H \varphi$. Now, however, it follows from Lemma 8.1 and the definition of $\mathcal{L}$ that

$$
C_{c}^{2} \subseteq\left(\lambda I+H_{p}\right)\left(L_{1 ; 2} \cap L_{\infty ; 2}\right)
$$

As $C_{c}^{2}$ is dense in $L_{p}$ this implies that $L_{1 ; 2} \cap L_{\infty ; 2}$ is a core for $H_{p}$. Thus $H_{p}$ is the $L_{p}$-closure of $H$ on $L_{p ; 2}$.

Next the $L_{\hat{1}}$-closure $\hat{H}_{1}$ of $H$ generates a continuous semigroup $\hat{S}^{(1)}$ on $L_{\hat{1}}$ which coincides with $S^{(\infty)}$ on $L_{\infty}$, by Proposition 7.2. Since $\|\varphi\|_{\hat{1}}=\left\|\triangle^{-1} \varphi\right\|_{1}$ for $\varphi$ with compact support and since $g \mapsto \Delta(g)^{-1}$ grows at most exponentially the proof of Lemma 8.1 establishes that

$$
\mathcal{L} \subseteq \mathcal{L}_{\hat{1} ; 2} \cap \mathcal{L}_{\infty ; 2}
$$

Hence the construction of $\hat{S}^{(p)}$ on $L_{\hat{p}}$ is identical to the foregoing construction of $S^{(p)}$ on $L_{p}$.

Finally; $S^{(p)}$ coincides with $S^{(\infty)}$ on $L_{p} \cap L_{\infty}$ and $\hat{S}^{(p)}$ coincides with $S^{(\infty)}$ on $L_{\hat{p}} \cap L_{\infty}$. Therefore $S^{(p)}$ and $\hat{S}^{(p)}$ coincide on $L_{p} \cap L_{\hat{p}} \cap L_{\infty}$. But $L_{p} \cap L_{\hat{p}}$ is a Banach space with respect to the norm

$$
\|\mid \varphi\|_{p}=\left(\int_{G} d g\left(1+\Delta(g)^{-1}\right)|\varphi(g)|^{p}\right)^{1 / p}
$$

and if $p \in[1, \infty\rangle$ it is clear that $L_{p} \cap L_{\hat{p}} \cap L_{\infty}$ is dense in $L_{p} \cap L_{\hat{p}}$. Therefore for each $\varphi \in L_{p} \cap L_{\hat{p}}$ there is a sequence $\varphi_{n} \in L_{p} \cap L_{\hat{p}} \cap L_{\infty}$ which is $\|\cdot\|_{p^{-}}$, and $\|\cdot\|_{\hat{p}^{-}}$, convergent to $\varphi$. Thus

$$
S_{t}^{(p)} \varphi=\lim _{n \rightarrow \infty} S_{t}^{(p)} \varphi_{n}=\lim _{n \rightarrow \infty} \hat{S}_{t}^{(p)} \varphi_{n}=\hat{S}_{t}^{(p)} \varphi
$$

and the semigroups are consistent. 


\section{Holomorphy}

In the previous section we constructed consistent interpolating semigroups $S$, and $\hat{S}$, generated by a subelliptic operator with smooth coefficients on the $L_{p^{-}}$, and $L_{\hat{p}^{-}}$, spaces. Next we argue that each $S^{(p)}$, and hence each $\hat{S}^{(p)}$, is holomorphic with a sector of holomorphy which is independent of $\mathrm{p}$. Since there are several variations in the meaning of 'holomorphic semigroup' we begin by stating the following known theorem $[\mathrm{Paz}][\mathrm{ReS}]$ which contains the definition we adopt and also gives useful criteria for holomorphy. In this theorem $t \mapsto T(t)=e^{-t H}$ is a strongly continuous semigroup on the Banach space $\mathcal{X}$, or a weakly* continuous semigroup if $\mathcal{X}$ has a predual.

Theorem 9.1 The following conditions are equivalent;

1. There exists a $\delta>0$ such that the function $t \in \mathbf{R}_{+} \mapsto T(t)$ extends to an analytic function

$$
z \in \Delta_{\delta}=\{z \in \mathbf{C} ;|\arg z|<\delta\} \mapsto T(z)
$$

with the properties

$$
\lim _{\substack{z \rightarrow 0 \\ z \in \Delta_{\delta}}} T(z) x=x
$$

for all $x \in \mathcal{X}$

$$
T\left(z_{1}+z_{2}\right)=T\left(z_{1}\right) T\left(z_{2}\right)
$$

for all $z_{1}, z_{2} \in \Delta$, and there is an $\omega \geq 0$ such that $\|T(z)\| e^{-\omega \operatorname{Rez}}$ is uniformly bounded in each closed subsector $\bar{\Delta}_{\delta^{\prime}}$ where $\delta^{\prime}<\delta$.

2. There exists $a \delta>0$ and an $\omega \geq 0$ such that for each $0<\delta^{\prime}<\delta$ there exists a constant $M_{\delta^{\prime}}>0$ and

$$
\left\|(\lambda I+H)^{-1}\right\| \leq M_{\delta^{\prime}} /|\lambda-\omega|
$$

for $\lambda-\omega \in \Delta_{\pi / 2+\delta^{\prime}}$, i.e., the resolvent exists and satisfies the estimate. 
3. $T(t) \mathcal{X} \subseteq D(H)$ whenever $t>0$ and there exists a constant $C$ such that

$$
\|H T(t)\| \leq C / t
$$

for sufficiently small $t$.

Furthermore, if any of these conditions are fulfilled, then the $\delta>0$ in Conditions 1 and 2 can be taken to be the same; if Condition 2 holds then $C$ in Condition 3 can be taken to be $M_{\delta^{\prime}} / \pi \cos \delta^{\prime}$; if Condition 3 holds we may take $\delta=\arctan (1 / C e)$ in Conditions 1 and 2.

The proof of the theorem follows from the discussion in [Paz] and [ReS]. Our aim is to prove that the interpolating semigroup constructed in Section 8 satisfies the condition of the theorem on each $L_{p}$-space with a $\delta$ which is independent of $p$. The key to this is the following result in which we use the notation

$$
\Delta_{\delta}^{\lambda_{0}}=\Delta_{\delta} \cap\left\{\lambda \in \mathrm{C} ;|\lambda|>\lambda_{0}\right\}
$$

Lemma 9.2 Let $H$ be a pure second-order subelliptic operator,

$$
H=\sum_{\alpha ;|\alpha|=2} c_{\alpha} A^{\alpha}
$$

on $L_{\infty}$ with coefficients $c_{\alpha} \in L_{\infty ; 5}$. There exist constants $a, \lambda_{0}, \delta>0$ and bounded operators $R_{\lambda}$ and $Q_{\lambda}$ on $L_{\infty}$ satisfying

$$
(\lambda I+H) R_{\lambda}=I+Q_{\lambda}
$$

and

$$
\left\|R_{\lambda}\right\|_{\infty \rightarrow \infty} \leq a|\lambda|^{-1}, \quad\left\|Q_{\lambda}\right\|_{\infty \rightarrow \infty} \leq a|\lambda|^{-1 / 2}
$$

for all $\lambda \in \Delta_{\pi / 2+\delta}^{\lambda_{0}}$.

Proof Let $K^{0}$ denote the semigroup kernel associated with the subelliptic operator

$$
H_{0}=-\sum_{\alpha ;|\alpha|=2} c_{\alpha}(g) A^{\alpha}
$$

with constant coefficients. Then $K^{0}$ satisfies the bounds (2.9). Consequently if $\partial_{t}=$ $\partial / \partial t$ then

$$
\left\|H_{0} K_{t}^{0}\right\|_{1}=\left\|\partial_{t} K_{t}^{0}\right\|_{1} \leq a t^{-1} e^{\rho t}
$$


for suitable $a>0$ and $\rho \geq 0$ by the estimation procedure used in the proof of Lemma 3.1. But using $K_{t+s}^{0}=K_{t}^{0} * K_{s}^{0}$ one deduces that

$$
\left\|\partial_{t}^{n} K_{t}^{0}\right\|_{1} \leq\left\|\partial_{t} K_{t / n}^{0}\right\|_{1}^{n} \leq n !\left(e a t^{-1}\right)^{n} e^{\rho t} .
$$

Thus the series

$$
z \mapsto \sum_{n \geq 0} \frac{z^{n}}{n !} \partial^{n} K_{t}^{0}
$$

converges in $L_{1}(G ; d g)$ for $|z|<t / a e$ and

$$
\left\|\sum_{n \geq 0} z^{n} \partial_{t}^{n} K_{t}\right\|_{1} \leq \sum_{n \geq 0}\left(|z| e a t^{-1}\right)^{n} e^{\rho t}
$$

Therefore defining $K_{t+z}^{0}$ by

$$
K_{t+z}^{0}=\sum_{n \geq 0} \frac{z^{n}}{n !} \partial_{t}^{n} K_{t}^{0}
$$

one concludes that $\left\|K_{t+z}\right\|_{1} e^{\rho t}$ is uniformly bounded in any sector $\{t+z ; t>0,|z|<$ $(1-\varepsilon) t / a e\}$ with $\varepsilon \in\langle 0,1]$. Thus if $H_{0}$ is viewed as an operator on any Banach space $\mathcal{X}$ where $G$ acts by an isometric representation $U$ then $H_{0}$ generates a holomorphic semigroup

$$
S_{t}=\int_{G} d h K_{t}^{0}(h) U(h)
$$

with holomorphy sector $\Delta_{\delta}$ where $\sin \delta=(a e)^{-1}$.

In particular this means that $e^{i \theta} H_{0}$ generates a semigroup with kernel $K_{e^{i \theta} t}$ whenever $|\theta|<\arcsin \lambda(a e)^{-1}$.

Now we are prepared to prove estimates analogous to those of Lemma 4.1 for complex $\lambda$. We again use the representation

$$
A_{i_{1}} \ldots A_{i_{m}}\left(\lambda I+e^{i \theta} H_{0}\right)^{-z}=\Gamma(z)^{-1} \int_{0}^{\infty} d t e^{-\lambda t} t^{z-1} A_{i_{1}} \ldots A_{i_{m}} S_{e^{i \theta} t}
$$

where $\operatorname{Re} \lambda>0$. Next set

$$
S_{e^{i \theta_{t}}}=S_{t / 2} \sum_{n \geq 0} \frac{z_{\theta}^{n}}{n !}(-H)^{n} S_{t / 2}
$$

where $z_{\theta}=\left(e^{i \theta}-1\right) t$. Thus

$$
\begin{aligned}
\| A_{i_{1}} \ldots A_{i_{n}}(\lambda I & \left.+e^{i \theta} H_{0}\right)^{-z} \| \\
& \leq|\Gamma(z)|^{-1} \int_{0}^{\infty} d t e^{-\operatorname{Re} \lambda t} t^{\operatorname{Re} z-1}\left\|A_{i_{1}} \ldots A_{i_{n}} K_{t / 2}^{0}\right\|_{1} \cdot \sum_{n \geq 0} \frac{\left|z_{\theta}\right|^{n}}{n !}\left\|H^{n} K_{t / 2}^{0}\right\|_{1} \\
& \leq|\Gamma(z)|^{-1} \sum_{n \geq 0} a_{m} \int_{0}^{\infty} d t e^{-\operatorname{Re} \lambda t} t^{\operatorname{Re} z-1} t^{-m / 2} e^{\rho t} \cdot\left(\left|z_{\theta}\right| e a t^{-1}\right)^{n} .
\end{aligned}
$$


Here we have used the estimate

$$
\left\|A_{i_{1}} \ldots A_{i_{m}} K_{t / 2}^{0}\right\|_{1} \leq a_{m} t^{-m / 2} e^{\rho t}
$$

which follows from (2.9). But if $|\theta|$ is small enough

$$
\sum_{n \geq 0}\left(\left|z_{\theta}\right| e a t^{-1}\right)^{n}=\sum_{n \geq 0}\left(\left|e^{i \theta}-1\right| e a\right)^{n}
$$

is bounded independently of $t$. Thus proceeding as in the proof of Lemma 4.1 we obtain estimates

$$
\left\|A_{i_{1}} \ldots A_{i_{m}}\left(\lambda I+e^{i \theta} H_{0}\right)^{-z}\right\|_{\infty \rightarrow \infty} \leq a_{m} \frac{\Gamma(\operatorname{Re} z-m / 2)}{|\Gamma(z)|}(\operatorname{Re} \lambda)^{m / 2-\operatorname{Re} z}
$$

for $|\theta|<\delta$ and $\operatorname{Re} \lambda>\lambda_{0}$ where $\delta$ and $\lambda_{0}$ are suitable positive constants.

One can now repeat the arguments used in Section 4 to establish Lemma 4.3 and obtain a generalization of this result in which $H_{0}$ is replaced by $e^{i \theta} H_{0}$ and the bounds $\lambda^{-1}, \lambda^{-1 / 2}$, are replaced by $(\operatorname{Re} \lambda)^{-1},(\operatorname{Re} \lambda)^{-1 / 2}$. Then remarking that

$$
\left(\lambda I+e^{i \theta} H_{0}\right)^{-1}=e^{-i \theta}\left(e^{-i \theta} \lambda I+H_{0}\right)^{-1}
$$

one deduces the following version of Lemma 4.3.

Lemma 9.3 There exist $a, \lambda_{0}, \delta>0$ such that

$$
\begin{gathered}
\left\|\left(\lambda I+H_{0}\right)^{-1}\right\| \leq a|\lambda|^{-1} \\
\left\|\left(\lambda I+H_{0}\right)^{-1} A_{i_{1}} A_{i_{2}}\left(\lambda I+H_{0}\right)^{-1}\right\| \leq a|\lambda|^{-1} \\
\left\|\left(\lambda I+H_{0}\right)^{-1} A_{i_{1}} A_{i_{2}}\left(\lambda I+H_{0}\right)^{-1} A_{i_{3}} A_{i_{4}}\left(\lambda I+H_{0}\right)^{-1}\right\| \leq a|\lambda|^{-1} \\
\left\|A_{i_{1}}\left(\lambda I+H_{0}\right)^{-1}\right\| \leq a|\lambda|^{-1 / 2} \\
\left\|A_{i_{1}}\left(\lambda I+H_{0}\right)^{-1} A_{i_{2}} A_{i_{3}}\left(\lambda I+H_{0}\right)^{-1}\right\| \leq a|\lambda|^{-1 / 2}
\end{gathered}
$$

for all $\lambda \in \Delta_{\pi / 2+\delta}^{\lambda_{0}}$ and $1 \leq i_{k} \leq d^{\prime}$.

Using these estimates one can now develop the parametrix method for $H$ exactly as in Section 5. For example, one defines

$$
\left(R_{\lambda} \varphi\right)(g)=\int_{G} d h \psi(h) R_{\lambda}(h ; g)(L(h) \varphi)(g)
$$


where $R_{\lambda}(-; g)$ is the kernel of the resolvent $\left(\lambda I+H_{0}\right)^{-1}$ and $\lambda \in \Delta_{\pi / 2+\delta}^{\lambda_{0}}$. This kernel can be obtained from the semigroup kernel $K^{0}$ corresponding to $H_{0}$ even if $\lambda$ is complex. Then

$$
R_{e^{i \theta|\lambda|}}(h ; g)=e^{-i \theta} \int_{0}^{\infty} d t e^{|\lambda| t} K_{e^{i \theta} t}^{0}(h)
$$

for small enough $\theta$. The proof of Lemma 9.2 is then completed by repetition of the arguments of Section 5 .

The parametrix $R_{\lambda}$, the remainder $Q_{\lambda}$, and the foregoing estimates now allow one to deduce holomorphy properties.

Lemma 9.4 The semigroup generated by the closure of the subelliptic operator $H$ of Lemma 9.2 is holomorphic on $L_{\infty}$.

Proof The basic parametrix formula

$$
\left(\psi,(\lambda I+H) R_{\lambda} \varphi\right)=\left(\psi,\left(I+Q_{\lambda}\right) \psi\right)
$$

for $\psi \in L_{1}, \varphi \in L_{\infty}$ and $\lambda \in \Delta_{\pi / 2+\delta}^{\lambda_{0}}$ gives the bounds

$$
\left\|\left(\lambda I+H^{*}\right) \psi\right\|_{1}\left\|R_{\lambda}\right\|_{\infty \rightarrow \infty}\|\varphi\|_{\infty} \geq|(\psi, \varphi)|-\|\psi\|_{1}\left\|Q_{\lambda}\right\|_{\infty \rightarrow \infty}\|\varphi\|_{\infty}
$$

for $\psi \in D\left(H^{*}\right)$. Now choosing $\varphi \in L_{\infty}$ such that $\|\varphi\|_{\infty}=1$ and $|(\psi, \varphi)|=\|\psi\|_{1}$ we obtain the estimates

$$
\left\|\left(\lambda I+H^{*}\right) \psi\right\|_{1} \geq a^{-1}\left(1-a\left|\lambda_{0}\right|^{-1 / 2}\right)|\lambda| \cdot\|\psi\|_{1}
$$

by use of the bounds in Lemma 9.2. Therefore the semigroup $S^{*}$ generated by $H^{*}$ on $L_{1}$ is holomorphic in the sector $\Delta_{\delta}$ and by duality the semigroup $S$ generated by the closure of $H$ on $L_{\infty}$ is holomorphic in the same sector.

Although this result is only established for pure second-order operators it extends to operators

$$
H=\sum_{\alpha ;|\alpha| \leq 2} c_{\alpha} A^{\alpha}
$$

with $c_{\alpha} \in L_{\infty ; 5}$ for $|\alpha|=2$ and $c_{\alpha} \in L_{\infty}$ for $|\alpha|<2$ by perturbation theory. Let $H_{0}$ now denote the operator of Lemma 9.2. The holomorphy of the corresponding semigroup on $L_{\infty}$ states that

$$
\left\|\left(\lambda I+H_{0}\right) \varphi\right\|_{\infty} \geq m_{\delta}|\lambda| \cdot\|\varphi\|_{\infty}
$$


for some $m_{\delta}$ and all $\varphi \in D\left(H_{0}\right), \lambda \in \Delta_{\pi / 2+\delta}^{\lambda_{0}}$. On the other hand it follows from the perturbation estimate (6.3) that there is an $a>0$ such that

$$
\left\|\left(H-H_{0}\right) \varphi\right\|_{\infty} \leq \varepsilon\left\|H_{0} \varphi\right\|_{\infty}+a \varepsilon^{-1}\|\varphi\|_{\infty}
$$

for all $\varphi \in D\left(H_{0}\right)=D(H)$ and $\varepsilon \in\langle 0,1]$. Therefore

$$
\begin{aligned}
\|(\lambda I+H) \varphi\|_{\infty} & \geq\left\|\left(\lambda I+H_{0}\right) \varphi\right\|_{\infty}-\left\|\left(H-H_{0}\right) \varphi\right\|_{\infty} \\
& \geq(1-\varepsilon)\left\|\left(\lambda I+H_{0}\right) \varphi\right\|_{\infty}-\left(\varepsilon|\lambda|+a \varepsilon^{-1}\right)\|\varphi\|_{\infty} \\
& \geq\left((1-\varepsilon) m_{\delta}-\varepsilon-a(\varepsilon|\lambda|)^{-1}\right)|\lambda| \cdot\|\varphi\|_{\infty}
\end{aligned}
$$

for all $\varepsilon \in\langle 0,1]$ and $\lambda \in \Delta_{\pi / 2+\delta}^{\lambda_{0}}$. Now we may assume $\lambda_{0}>1$ and set $\varepsilon=|\lambda|^{-1 / 2} \leq$ $\left|\lambda_{0}\right|^{-1 / 2}$. This then gives

$$
\|(\lambda I+H) \varphi\|_{\infty} \geq m_{\delta}^{\prime}|\lambda| \cdot\|\varphi\|_{\infty}
$$

with $m_{\delta}^{\prime}=\left(1-\left|\lambda_{0}\right|^{-1 / 2}\right) m_{\delta}-\left|\lambda_{0}\right|^{-1 / 2}(1+a)$. Increasing the value of $\lambda_{0}$ if necessary one then has $m_{\delta}^{\prime}>0$ and the resolvent of $H$ satisfies the bounds of Condition 2 in Theorem 9.1. Therefore the corresponding semigroup is holomorphic in the sector $\Delta_{\delta}$.

This last result is the key to proving that the interpolating semigroup of Proposition 8.2 is holomorphic on each of the $L_{p}$-spaces.

Proposition 9.5 If $c_{i j} \in L_{\infty ; 5}, c_{i} \in L_{\infty ; 4}$, and $c_{0} \in L_{\infty}$, then the semigroup $S^{(p)}$ $\left(\hat{S}^{(p)}\right)$ generated by the $L_{p}$-closure ( $L_{\hat{p}}$-closure) of the subelliptic operator (1.20) is holomorphic in a sector $\Delta_{\delta}$ with $\delta>0$ independent of $p$.

Proof The foregoing argument establishes that $S^{(\infty)}$ is holomorphic in a sector $\Delta_{\delta}$. But the same argument applies to the formal adjoint (1.23) and in fact the corresponding semigroup $S^{t(\infty)}$ is holomorphic in the same sector $\Delta_{\delta}$. Now in Section 7 we argued that $H^{\dagger *}=H$, the closed operator on $L_{1}$. Therefore $S^{(1)}$ is holomorphic in $\Delta_{\delta}$. Then it follows from the Riesz-Thorin

interpolation theorem that $S^{(p)}$ is holomorphic in $\Delta_{\delta}$. For example, holomorphy on $L_{\hat{1}}$ and $L_{\infty}$ is equivalent to resolvent bounds

$$
\left\|(\lambda I+H)^{-1}\right\|_{\hat{1} \rightarrow \hat{1}} \leq M_{\delta}|\lambda|^{-1},\left\|(\lambda I+H)^{-1}\right\|_{\infty \rightarrow \infty} \leq M_{\delta}|\lambda|^{-1}
$$

for $\lambda \in \Delta_{\pi / 2+\delta}^{\delta_{0}}$ and these bounds extend to $L_{p}$ by interpolation. Finally $\hat{S}^{(p)}$ is holomorphic in the same sector because $\mathrm{S}$ and $\hat{S}$ are consistent. 
Remark -Although the holomorphy angle $\delta$ determined by the foregoing estimates is independent of $p$ it does depend on the ellipticity constant and the $C^{n}$-norms $\left\|c_{i j}\right\|_{\infty ; 5}$ etc. of the coefficients. The estimates of Corollary 10.2 will, however, establish the existence of a holomorphy sector $\Delta_{\delta}$ determined by $\lambda_{c}$ and the norms $\left\|c_{i j}\right\|_{\infty},\left\|c_{i}\right\|_{\infty},\left\|c_{0}\right\|_{\infty}$, i.e., independent of $p$ and of the derivatives of the coefficients. 


\section{Semigroup kernels}

Throughout this section we assume that $H$ is a (closed) subelliptic operator in the form

$$
H=-\sum_{i, j=1}^{d^{\prime}} A_{i} c_{i j} A_{j}+\sum_{i=1}^{d^{\prime}}\left(c_{i} A_{i}+A_{i} c_{i}^{\prime}\right) / 2+c_{0} .
$$

Thus we simultaneously discuss each of the forms (1.20)-(1.22). We assume that $c_{i j} \in L_{\infty ; 5}, c_{i}, c_{i}^{\prime} \in L_{\infty ; 4}$ and $c_{0} \in L_{\infty}$. Then $H$ generates interpolating semigroups $S$, and $\hat{S}$, on the $L_{p^{-}}$and $L_{\hat{p}^{-}}$, spaces as a consequence of Proposition 8.2. Next we argue that $S$ is given by an integral kernel $K$ through (1.30) and obtain upper bounds on the kernel and its derivatives with respect to $t$ and $g$.

The bounds we obtain differ in character from all the estimates of Sections 6-9. Those estimates were based on parametrix arguments and the bounds depend on the coefficients of $H$ through the ellipticity constant $\lambda_{c}$, defined by (1.18), and the norms $\left\|c_{i j}\right\|_{\infty ; 5},\left\|c_{i}\right\|_{\infty ; 4},\left\|c_{i}^{\prime}\right\|_{\infty ; 4},\left\|c_{0}\right\|_{\infty}$. But the upper bounds on the kernel will only depend on $\lambda_{c},\left\|c_{i j}\right\|_{\infty},\left\|c_{i}\right\|_{\infty},\left\|c_{i}^{\prime}\right\|_{\infty}$ and $\left\|c_{0}\right\|_{\infty}$, i.e., the derivatives of the coefficients do not occur. This is important for the subsequent extension to the operators with non-smooth coefficients. This greater flexibility arises because the bounds are derived by $L_{2}$-estimates which are much easier to handle than the previous $L_{\infty}$-estimates.

The existence of the kernel $K$ follows from the Dunford-Pettis theorem (see, for example, [Tre] or [CFKS]) once one establishes that the interpolating semigroup $\hat{S}$ is bounded from $L_{\hat{1}}$ to $L_{\infty}$. Since $\hat{S}$ is bounded from $L_{\infty}$ to $L_{\infty}$ it follows by interpolation that it is bounded from $L_{\hat{p}}$ to $L_{\infty}$ and

$$
\left\|\hat{S}_{t}\right\|_{\hat{p} \rightarrow \infty}=\left\||| K_{t}\right\| \|_{\hat{q}}=\underset{g \in G}{\operatorname{ess} \sup _{G}}\left(\int_{G} d \hat{h}\left|K_{t}(g ; h)\right|^{q}\right)^{1 / q}
$$

where $q$ is the dual variable to $p$. Although the Dunford-Pettis theorem presupposes some separability this can be avoided by use of the Lie group structure (see [BrR1] Section 2).

Next we establish the boundedness of $\left\|S_{t}\right\|_{\hat{1} \rightarrow \infty}$ for $t>0$ by a differential inequality combined with a Nash inequality as in [CKS] [FaS] [Rob1] [Rob2] or [BrR1]. 
First we use the a priori inequality on $L_{\hat{2}}$ of Proposition 3.1, i.e., the inequality

$$
\left\|A_{i} \varphi\right\|_{\hat{2}} \leq \varepsilon\|H \varphi\|_{\hat{2}}+a \varepsilon^{-1}\|\varphi\|_{\hat{2}}
$$

for all $\varepsilon \in\langle 0,1]$ and $\varphi \in L_{\hat{2} ; 2}^{\prime}$. The bounds (10.2) then extend by closure to all $\varphi \in D\left(H_{\hat{2}}\right)$ where $H_{\hat{2}}$ is the $L_{\hat{2}}$-closure of $H$ and this establishes that $D\left(H_{\hat{2}}\right) \subseteq L_{\hat{2} ; 1}$. Moreover, $\left\|A_{i}\left(\lambda I+H_{\hat{2}}\right)^{-1}\right\|_{\hat{2} \rightarrow \hat{2}}<\infty$ for all $\lambda>0$. But $\left\|A_{i}\left(\lambda I+H_{\infty}\right)^{-1}\right\|_{\infty \rightarrow \infty}<\infty$ with $H_{\infty}$ the $L_{\infty}$-closure of $H$ by Proposition 6.4. Therefore $\left\|A_{i}\left(\lambda I+H_{\hat{p}}\right)^{-1}\right\|_{\hat{p} \rightarrow \hat{p}}<\infty$

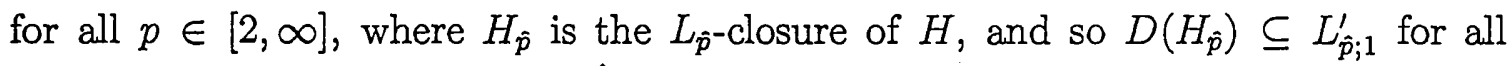
$p \in[2, \infty]$. Since the semigroup $\hat{S}^{(p)}$ generated by $H_{\hat{p}}$ is holomorphic by Proposition 9.5 it then follows that

$$
\hat{S}_{t}^{(p)} L_{\hat{p}} \subseteq D\left(H_{\hat{p}}\right) \subseteq L_{\hat{p} ; 1}^{\prime}
$$

for all $t>0$ and $p \in[2, \infty]$. This regularity property justifies the differential techniques used in the subsequent arguments.

Following Fabes and Stroock $[\mathrm{FaS}]$ we bound $\left\|\hat{S}_{t}\right\|_{\hat{2} \rightarrow \infty}$ by obtaining a succession of bounds on $\left\|\hat{S}_{t}\right\|_{\hat{2} \rightarrow \hat{4}},\left\|\hat{S}_{t}\right\|_{\hat{4} \rightarrow \hat{8}}$, etc. The starting point of the method is a bound on $\left\|\hat{S}_{t}\right\|_{\hat{2} \rightarrow \hat{2}}$. First note that

$$
\begin{aligned}
\frac{d}{d t}\left\|S_{t} \varphi\right\|_{2}^{2} & =-2 \operatorname{Re}\left(S_{t} \varphi, H S_{t} \varphi\right) \\
\leq & -2 \lambda_{c} \sum_{i=1}^{d^{\prime}}\left\|A_{i} S_{t} \varphi\right\|_{2}^{2}+\sum_{i=1}^{d^{\prime}}\left(\left\|c_{i}\right\|_{\infty}+\left\|c_{i}^{\prime}\right\|_{\infty}\right)\left\|S_{t} \varphi\right\|_{2}\left\|A_{i} S_{t} \varphi\right\|_{2}+2\left\|c_{0}\right\|_{\infty}\left\|S_{t} \varphi\right\|_{2}^{2}
\end{aligned}
$$

where we use the simplified notation $S=S^{(p)}, \hat{S}=\hat{S}^{(p)}$ and $H=H_{\hat{p}}$. But

$$
\sum_{i=1}^{d^{\prime}}\left\|c_{i}\right\|_{\infty}\left\|S_{t} \varphi\right\|_{2}\left\|A_{i} S_{t} \varphi\right\|_{2} \leq \varepsilon \sum_{i=1}^{d^{\prime}}\left\|A_{i} S_{t} \varphi\right\|_{2}^{2}+(4 \varepsilon)^{-1} \sum_{i=1}^{d^{\prime}}\left\|c_{i}\right\|_{\infty}^{2}\left\|S_{t} \varphi\right\|_{2}^{2}
$$

Therefore, setting $\varepsilon=\lambda_{c}$,

$$
\frac{d}{d t}\left\|S_{t} \varphi\right\|_{2}^{2} \leq \omega\left\|S_{t} \varphi\right\|_{2}^{2}
$$

with $\omega=2\left\|c_{0}\right\|_{\infty}+\sum_{i=1}^{d^{\prime}}\left(\left\|c_{i}\right\|_{\infty}^{2}+\left\|c_{i}^{\prime}\right\|_{\infty}^{2}\right) / 4 \lambda_{c}$ and by integration

$$
\left\|S_{t}\right\|_{2 \rightarrow 2} \leq e^{\omega t} \text {. }
$$

But

$$
\left\|\hat{S}_{t}\right\|_{\hat{2} \rightarrow \hat{2}}=\left\|\Delta^{-1 / 2} S_{t} \Delta^{1 / 2}\right\|_{2 \rightarrow 2}
$$


and $\Delta^{-1 / 2} S_{t} \Delta^{1 / 2}$ is the semigroup generated by the operator $\Delta^{-1 / 2} H \Delta^{1 / 2}$ which is a subelliptic operator of the same type as $H$ with coefficients which are linear combinations of the coefficients of $H$. Therefore one has bounds

$$
\left\|\hat{S}_{t}\right\|_{\hat{2} \rightarrow \hat{2}} \leq e^{\bar{\omega} t}
$$

where the value of $\hat{\omega}$ is determined by the norm

$$
c \mapsto\||c|\|=\sum_{i, j=1}^{d^{\prime}}\left\|c_{i j}\right\|_{\infty}+\sum_{i=1}^{d^{\prime}}\left(\left\|c_{i}\right\|_{\infty}+\left\|c_{i}^{\prime}\right\|_{\infty}\right)+\left\|c_{0}\right\|_{\infty}
$$

and $\lambda_{c}$.

Next let $\varphi \in L_{1} \cap L_{\infty}$. Then $S_{t} \varphi \in L_{p ; 1}^{\prime}$ for all $p \in[1, \infty]$. Hence $H S_{t} \varphi \in L_{2}$ and $\left(S_{t} \varphi\right)^{n} \in L_{2}$ for $n=1,2, \ldots$ Therefore if $p=2^{n}$ then

$$
\frac{d}{d t}\left\|S_{t} \varphi\right\|_{2 p}=-\left\|S_{t} \varphi\right\|_{2 p}^{1-2 p}\left(\left(S_{t} \varphi\right)^{2 p-1}, H S_{t} \varphi\right)
$$

Now the second-order term of $H$ gives a contribution

$$
\begin{aligned}
\sum_{i, j=1}^{d^{\prime}}\left(A_{i}\left(S_{t} \varphi\right)^{2 p-1}, c_{i j} A_{j} S_{t} \varphi\right) & =\left((2 p-1) / p^{2}\right) \sum_{i, j=1}^{d^{\prime}}\left(A_{i}\left(S_{t} \varphi\right)^{p}, c_{i j} A_{j}\left(S_{t} \varphi\right)^{p}\right) \\
& \geq \lambda_{c}\left((2 p-1) / p^{2}\right) \sum_{i=1}^{d^{\prime}}\left\|A_{i}\left(S_{t} \varphi\right)^{p}\right\|_{2}^{2}
\end{aligned}
$$

whilst the first-order term contributes

$$
\begin{aligned}
\sum_{i=1}^{d^{\prime}}\left(\left(c_{i}\left(S_{t} \varphi\right)^{2 p-1}, A_{i} S_{t} \varphi\right)-\left(A_{i}\left(S_{t} \varphi\right)^{2 p-1}, c_{i}^{\prime} S_{t} \varphi\right)\right) / 2 & \\
& \left.=\sum_{i=1}^{d^{\prime}}\left(\left(c_{i} S_{t} \varphi\right)^{p}, A_{i}\left(S_{t} \varphi\right)^{p}\right) / p-\left(A_{i}\left(S_{t} \varphi\right)^{p}, c_{i}^{\prime}\left(S_{t} \varphi\right)^{p}\right)(2 p-1) / p\right) / 2 \\
& \geq-\left(\|c\|_{\infty}+\left\|c^{\prime}\right\|_{\infty}\right)\left(\sum_{i=1}^{d^{\prime}}\left\|A_{i}\left(S_{t} \varphi\right)^{p}\right\|_{2}^{2}\right)^{1 / 2}\left\|\left(S_{t} \varphi\right)^{p}\right\|_{2} \\
& \geq-\varepsilon \sum_{i=1}^{d^{\prime}}\left\|A_{i}\left(S_{t} \varphi\right)^{p}\right\|_{2}^{2}-(4 \varepsilon)^{-1}\left(\|c\|_{\infty}+\left\|c^{\prime}\right\|_{\infty}\right)^{2}\left\|S_{t} \varphi\right\|_{2 p}^{2 p}
\end{aligned}
$$

where $\varepsilon>0$ and $\|c\|_{\infty}=\left(\sum_{i=1}^{d^{\prime}}\left\|c_{i}\right\|_{\infty}^{2}\right)^{1 / 2}$. Finally the zero-order term of $H$ contributes

$$
\left(\left(S_{t} \varphi\right)^{2 p-1}, c_{0} S_{t} \varphi\right) \geq-\left\|c_{0}\right\|_{\infty}\left\|S_{t} \varphi\right\|_{2 p}^{2 p}
$$


Consequently, if one combines these estimates with (10.5), sets $\varepsilon=\lambda_{c}(2 p-1) / 2 p^{2}$ and uses $2 p-1 \geq p$ one obtains the differential inequality

$$
\frac{d}{d t}\left\|S_{t} \varphi\right\|_{2 p} \leq-\left(\lambda_{c} / p\right)\left\|S_{t} \varphi\right\|_{2 p}^{1-2 p} \sum_{i=1}^{d^{\prime}}\left\|A_{i}\left(S_{t} \varphi\right)^{p}\right\|_{2}^{2}+p \mu\left\|S_{t} \varphi\right\|_{2 p}
$$

with $\mu=\left(\|c\|_{\infty}+\left\|c^{\prime}\right\|_{\infty}\right)^{2} / 2 \lambda_{c}+\left\|c_{0}\right\|_{\infty}$.

This differential inequality can be converted into a similar inequality for $\left\|\hat{S}_{t} \varphi\right\|_{\hat{2 p}}$ by noting that

$$
\left\|\hat{S}_{t} \varphi\right\|_{2 p}=\left\|\left(\Delta^{-1 / 2 p} \hat{S}_{t} \Delta^{1 / 2 p}\right) \Delta^{-1 / 2 p} \varphi\right\|_{2 p}
$$

and $\Delta^{-1 / 2 p} S_{t} \Delta^{1 / 2 p}$ is the semigroup on $L_{2 p}$ generated by the second-order subelliptic operator $\Delta^{-1 / 2 p} H \Delta^{1 / 2 p}$. This operator has the same second-order part as $H$ but the first-order and zero-order coefficients are linear combinations of the coefficients of $H$. Therefore-(10.6) gives

$$
\frac{d}{d t}\left\|\hat{S}_{t} \varphi\right\|_{\hat{2 p}} \leq-\left(\lambda_{c} / p\right)\left\|\hat{S}_{t} \varphi\right\|_{2 p}^{1-2 p} \sum_{i=1}^{d^{\prime}}\left\|A_{i} \Delta^{-1 / 2}\left(\hat{S}_{t} \varphi\right)^{p}\right\|_{2}^{2}+p \mu\left\|\hat{S}_{t} \varphi\right\|_{\hat{2 p}}
$$

where $\mu$ is an increasing function of $\||c|\|$ and a decreasing function of $\lambda_{c}$. At this point we use the Nash inequalities (IV.4.29) of [Rob1]. Specifically

$$
\|\psi\|_{\hat{2}} \leq K\left(\Gamma\left(\Delta^{-1 / 2} \psi\right) /\|\psi\|_{\hat{1}}\right)^{D^{\prime} /\left(2+D^{\prime}\right)}\|\psi\|_{\hat{1}}
$$

for all $\psi \in L_{\hat{1}} \cap L_{\hat{2} ; 1}^{\prime}$ where $K$ is a constant and

$$
\Gamma(\psi)=\left(\sum_{i=1}^{d^{\prime}}\left\|A_{i} \psi\right\|_{2}^{2}+\|\psi\|_{2}^{2}\right)^{1 / 2}
$$

Now one can combine (10.7) and (10.8), with $\psi=\left(\hat{S}_{t} \varphi\right)^{p}$, to obtain a final differential inequality

$$
\frac{d}{d t}\left\|\hat{S}_{t} \varphi\right\|_{2 p} \leq-\left(\lambda_{c} / p\right) K^{-4 / D^{\prime}-2}\left\|\hat{S}_{t} \varphi\right\|_{2 p}^{1+4 p / D^{\prime}}\left\|\hat{S}_{t} \varphi\right\|_{\hat{p}}^{-4 p / D^{\prime}}+p \hat{\mu}\left\|\hat{S}_{t} \varphi\right\|_{2 p}
$$

with $\hat{\mu}=\mu+\lambda_{c}$. But this is exactly the same form as the inequality (1.2) of Fabes and Stroock [FaS] and their solution gives bounds

$$
\left\|\hat{S}_{t}\right\|_{\hat{2} \rightarrow \infty} \leq a\left(\lambda_{c} t\right)^{-D^{\prime} / 4} e^{2 \hat{\mu} t}
$$

where $a>0$ is independent of the coefficients. Now

$$
\left\|\hat{S}_{t}\right\|_{\hat{1} \rightarrow \hat{2}}=\left\|\Delta S_{t}^{\dagger} \Delta^{-1}\right\|_{\hat{2} \rightarrow \infty}
$$


with $S_{t}^{\dagger}$ the semigroup generated by the formal adjoint $H^{\dagger}$, defined by (1.24), acting on the $L_{p}$-spaces. But $\Delta S_{t}^{\dagger} \Delta^{-1}$ is generated by $\Delta H^{\dagger} \Delta^{-1}$ and this is a subelliptic operator with second-order coefficients $c_{i j}$ and the other coefficients are again linear combinations of the coefficients of $H$. Therefore (10.11) applied to $\Delta S^{\dagger} \Delta^{-1}$ gives bounds

$$
\left\|\hat{S}_{t}\right\|_{\hat{1} \rightarrow \hat{2}} \leq a\left(\lambda_{c} t\right)^{-D^{\prime} / 4} c^{2 \hat{\nu} t}
$$

where $\hat{\nu}$ can be computed from $\hat{\mu}$ by replacing the coefficients of $H$ by the new coefficients. Then (10.11) and (10.12) give the final bounds

$$
\left\|\hat{S}_{t}\right\|_{\hat{i} \rightarrow \infty} \leq a_{1}\left(\lambda_{c} t\right)^{-D^{\prime} / 2} e^{\mu t}
$$

where $\mu$ depends on $\lambda_{c}$ and $\|c \mid\|$ as before.

At this point we have established that $\hat{S}$ is bounded as an operator from $L_{\hat{1}}$ to $L_{\infty}$. Hence it is given by a kernel $K$. Moreover, since $\left\|K_{t}\right\|_{\infty}=\left\|\hat{S}_{t}\right\|_{\hat{i} \rightarrow \infty}$ one has pointwise bounds on $K$ by (10.13). But these bounds can be converted into Gaussian bounds by Davies' method [Dav1]. This consists of applying the bounds (10.13) to the semigroups $\hat{S}_{t}^{\rho}=U_{\rho} \hat{S}_{t} U_{\rho}^{-1}$ where $U_{\rho}=e^{\rho \psi}$ is a family of multiplication operators with $\rho \in \mathbf{R}$ and $\psi \in C_{c}^{\infty}$ is chosen to satisfy

$$
\sum_{i=1}^{d^{\prime}}\left\|A_{i} \psi\right\|_{\infty}^{2} \leq 1
$$

Then $\hat{S}^{\rho}$ is generated by the subelliptic operator $H^{\rho}=U_{\rho} H U_{\rho}^{-1}$ with second-order coefficients $c_{i j}$, first-order $c_{i}+\rho \sum_{j=1}^{d^{\prime}} c_{i j}\left(A_{j} \psi\right), c_{i}^{\prime}+\rho \sum_{j=1}^{d^{\prime}} c_{i j}\left(A_{j} \psi\right)$ and zero-order $c_{0}+\rho \sum_{i=1}^{d^{\prime}}\left(c_{i}+c_{i}^{\prime}\right)\left(A_{i} \psi\right) / 2+\rho^{2} \sum_{i, j=1}^{d^{\prime}} c_{i j}\left(A_{i} \psi\right)\left(A_{j} \psi\right)$. Consequently (10.13) gives bounds

$$
\left\|S_{t}^{\rho}\right\|_{\hat{\mathrm{i}} \rightarrow \infty} \leq a_{1}\left(\lambda_{c} t\right)^{-D^{\prime} / 2} e^{\Lambda\left(1+\rho^{2} t\right)}
$$

where $\Lambda$ is independent of $\rho$. But this gives pointwise bounds on the kernel of $S^{\rho}$ which can be explicitly written as

$$
e^{\rho|\psi(g)-\psi(h)|}\left|K_{t}(g ; h)\right| \leq a_{1}\left(\lambda_{c} t\right)^{-D^{\prime} / 2} e^{\Lambda\left(1+\rho^{2}\right) t} .
$$

Now the choice of $\psi$ is such that [Dav1]

$$
\left|g h^{-1}\right|^{\prime}=\sup \left\{|\psi(g)-\psi(h)| ; \sum_{i=1}^{d^{\prime}}\left\|A_{i} \psi\right\|_{\infty}^{2} \leq 1\right\}
$$


and hence-one has bounds

$$
\begin{aligned}
\left|K_{t}(g ; h)\right| & \leq a_{1}\left(\lambda_{c} t\right)^{-D^{\prime} / 2} e^{\Lambda t} \inf _{\rho>0} e^{\Lambda \rho^{2} t-\rho\left|g h^{-1}\right|^{\prime}} \\
& =a_{1}\left(\lambda_{c} t\right)^{-D^{\prime} / 2} e^{\Lambda t} e^{-\left(\left|g h^{-1}\right|^{\prime}\right)^{2} / 4 \Lambda t} .
\end{aligned}
$$

The important point in the sequel is that $\Lambda$ depends on the coefficients of $H$ only through $\||c| \mid$ and $\lambda_{c}$. It is an increasing function of $\|c \mid\|$ and a decreasing function of $\lambda_{c}$. Moreover, $a_{1}$ is independent of the coefficients. Although it was necessary to assume some smoothness of the coefficients to construct $S$ and $K$ the bounds do not depend upon the derivatives of the coefficients.

In addition to the upper bounds it follows from the arguments of [ABR] that the kernel $K$ is positive. In Section 12 we will derive some detailed lower bounds but next we consider upper bounds on derivatives of the kernel.

First consider the derivatives $\partial_{t}^{n} K_{t}$ where $\partial_{t}=\partial / \partial t$. Then

$$
e^{\rho(\psi(g)-\psi(h))}\left|\left(\partial_{t}^{n} K_{t}\right)(g ; h)\right| \leq\left\|\left(H^{\rho}\right)^{n} \hat{S}_{t}^{\rho}\right\|_{\hat{i} \rightarrow \infty}
$$

with $H^{\rho}=U_{\rho} H U_{\rho}^{-1}$. But

$$
\left\|\left(H^{\rho}\right)^{n} \hat{S}_{t}^{\rho}\right\|_{\hat{1} \rightarrow \infty} \leq\left\|\left(H^{\rho}\right)^{n} \hat{S}_{t / 2}^{\rho}\right\|_{\hat{2} \rightarrow \hat{2}}\left\|\hat{S}_{t / 4}^{\rho}\right\|_{\hat{1} \rightarrow \hat{2}}\left\|\hat{S}_{t / 4}^{\rho}\right\|_{\hat{2} \rightarrow \infty} .
$$

The last two factors have already been bounded and following Davies [Dav2] we use the Cauchy integral representation to bound the first, i.e.,

$$
S_{t}^{\rho}=(2 \pi i)^{-1} \int_{C_{r}(t)} d z \frac{S_{z}^{\rho}}{z-t}
$$

where the integral is around a small circle $C_{r}(t)$ of radius $r$ centred at $t$. Therefore

$$
\left(H^{\rho}\right)^{n} S_{t}^{\rho}=(2 \pi i)^{-1} n ! \int_{C_{r}(t)} d z \frac{S_{z}^{\rho}}{(z-t)^{n+1}}
$$

Thus

$$
\left\|\left(H^{\rho}\right)^{n} S_{t}^{\rho}\right\|_{\hat{2} \rightarrow \hat{2}} \leq n ! r^{n} \sup \left\{\left\|S_{z}^{\rho}\right\|_{\hat{2} \rightarrow \hat{2}} ; z \in C_{r}(t)\right\}
$$

and the problem is reduced to bounding $S_{z}^{\rho}$ on $L_{\hat{2}}$ in terms of the norms on the coefficients. But this is straightforward and after minimizing with respect to $r$, using (10.16) and the bounds (10.11) and (10.12) applied to $S^{\rho}$, one obtains bounds

$$
\left\|\left(H^{\rho}\right)^{n} S_{t}^{\rho}\right\|_{\hat{1} \rightarrow \infty} \leq a b^{n} n ! t^{-n}\left(\lambda_{c} t\right)^{-D^{\prime} / 2} e^{\Lambda\left(1+\rho^{2}\right) t}
$$


analogous to (10.13). Then, however, this gives bounds on $\left|\left(\partial_{t}^{n} K_{t}\right)(g ; h)\right|$ which can be minimized with respect to $\rho$ to obtain Gaussian bounds. In summary these arguments give the following.

Proposition 10.1 If $c_{i j} \in L_{\infty ; 5}, c_{i}, c_{i}^{\prime} \in L_{\infty ; 4}$, and $c_{0} \in L_{\infty}$, then the semigroup $S$ generated by the corresponding subelliptic operator (10.1) has a positive integral kernel $K$. Moreover there are $a, b, \Lambda>0$ and $\omega \geq 0$ such that

$$
\begin{gathered}
0 \leq K_{t}(g ; h) \leq a\left(\lambda_{c} t\right)^{-D^{\prime} / 2} e^{\omega t} e^{-\left(\left|g h^{-1}\right|^{\prime}\right)^{2} / 4 \Lambda t} \\
\left|\left(\partial_{t}^{n} \cdot K_{t}\right)(g ; h)\right| \leq a b^{n} n ! t^{-n}\left(\lambda_{c} t\right)^{-D^{\prime} / 2} e^{\omega t} e^{-\left(\left|g h^{-1}\right|^{\prime}\right)^{2} / 4 \Lambda t}
\end{gathered}
$$

for all $g, h \in G, t>0$, and $n=1,2, \ldots$. The value of $a$ is independent of the coefficients of $H$ but $b, \Lambda$ and $\omega$ are increasing functions of $\||c|\|$ and decreasing functions of $\lambda_{c}$.

Remark If $H$ is in the asymmetric form (1.17) it can be re-expressed in the form (10.1) with $c_{i}^{\prime}=0$ and then the coefficients $c_{i j}, c_{i}, c_{0}$ are linear combinations of the $c_{\alpha}$ with $|\alpha|=0,1,2$ and the derivatives $A_{i} c_{\alpha}$ with $i=1, \ldots, d^{\prime}$ and $|\alpha|=2$. Therefore the bounds of Proposition 10.1 are still valid but $\Lambda$ is an increasing function of the norm $\|c\|_{1}^{\prime}$ defined by (3.4) and a decreasing function of $\lambda_{c}$.

The bounds of the proposition can be improved in several ways by more careful estimation. The details for constant coefficients are given in [Rob1] Chapter IV and the case of variable coefficients is not significantly more difficult. We discuss this at the end of the section but first we derive one useful corollary of the foregoing bounds.

Corollary 10.2 There exists an $a>0$ and an $\omega \geq 0$ which depend only on $\lambda_{c}$ and $\|$ |l $\|$ such that

$$
\begin{aligned}
\left\|S_{t}\right\|_{p \rightarrow p} & \leq a e^{\omega t}, \quad\left\|\hat{S}_{t}\right\|_{\hat{p} \rightarrow \hat{p}} \leq a e^{\omega t}, \\
\left\|H S_{t}\right\|_{p \rightarrow p} & \leq a t^{-1} e^{\omega t}, \quad\left\|H \hat{S}_{t}\right\|_{\hat{p} \rightarrow \hat{p}} \leq a t^{-1} e^{\omega t},
\end{aligned}
$$

for all $t>0$ and $p \in[1, \infty]$. In particular $S$ is holomorphic in an open sector $\Delta_{\delta}$ which is $p$-independent and determined by the parameters $\lambda_{c}$ and $\||c \||$.

Proof The proofs of all four bounds are very similar and hence we only consider the first. One has

$$
\left\|S_{t}\right\|_{\infty \rightarrow \infty}=\underset{g \in G}{\operatorname{ess} \sup _{\infty}} \int_{G} d \hat{h} K_{t}(g ; h)
$$


and

$$
\left\|S_{t}\right\|_{1 \rightarrow 1} \leq \operatorname{esssup}_{h \in G} \int_{G} d \hat{g} \Delta\left(g h^{-1}\right) K_{t}(g ; h)
$$

Now both these bounds can be estimated with the kernel bounds (10.18) and the procedure used in the proof of Lemma 4.1. For this it is important that the modular function grows at most exponentially, i.e., one has bounds $\Delta\left(g h^{-1}\right) \leq \alpha e^{\gamma\left|g h^{-1}\right|^{\prime}}$. The resulting estimates

$$
\left\|S_{t}\right\|_{\infty \rightarrow \infty} \leq a e^{\omega t} \quad, \quad\left\|S_{t}\right\|_{1 \rightarrow 1} \leq a e^{\omega t}
$$

can then be interpolated to obtain the $L_{p}$-estimates. Since the kernel bounds are independent of the derivatives of the coefficients the values of $a$ and $\omega$ are also independent of these derivatives.

Analyticity of the function $t \mapsto K_{t} \in \mathcal{L}_{\infty}$ now follows from holomorphy of $S$. First extend $S$ to $\mathcal{L}_{\infty}$ by setting

$$
\left(S_{t} \varphi\right)(g ; h)=\left(K_{t} * \varphi\right)(g ; h)=\int_{G} d k K_{t}(g ; k) \varphi(k ; h)
$$

Then the extended $S$ is automatically holomorphic in $\mathcal{L}_{\infty}$. But $K_{s} \in \mathcal{L}_{\infty}$ and $S_{t} K_{s}=$ $K_{t+s}$. Therefore $K$ is analytic in the sector $\Delta_{\delta}$ for which $S$ is holomorphic on $\mathcal{L}_{\infty}$.

One can also obtain bounds on the operators $A_{i} S_{t}$ and $A_{i} \hat{S}_{t}$, on the $L_{p^{-}}$, and $L_{\hat{p}^{-}}$, spaces but this requires an investigation of the left derivatives of the semigroup kernel. Since $K_{t} \in \mathcal{L}_{\hat{p}}$ for all $p \in[1, \infty]$ and $S_{t} K_{s}=K_{t+s}$ on $\mathcal{L}_{\hat{p}}$ and since $S_{t} L_{\infty} \subseteq L_{\infty ; 1}^{\prime}$ the kernel is once left differentiable, in the directions $a_{1}, \ldots, a_{d^{\prime}}$, with respect to the first variable. Now the kernel $K^{\dagger}$ associated with the formal adjoint is linked to $K$ by the symmetry property

$$
K_{t}(g ; h)=\Delta(g)^{-1} \Delta(h) K_{t}^{\dagger}(h ; g)
$$

Therefore $K_{t}$ must be differentiable in the $\mathcal{L}_{\infty}$-sense in the directions $a_{1}, \ldots, a_{d^{\prime}}$, with respect to both variables. Next we bound $A_{i} S_{t}$ and $A_{i} \hat{S}_{t}$ by use of the a priori estimates of Section 3 and bounds on $A_{i} K_{t}$, i.e., derivatives of $K_{t}$ with respect to the first variable.

First it follows from (3.12) that

$$
\left\|A_{i} S_{t} \varphi\right\|_{\infty} \leq \varepsilon\left\|H S_{t} \varphi\right\|_{\infty}+a_{\delta} \varepsilon^{-1-\delta}\left\|S_{t} \varphi\right\|_{\infty}
$$


Hence

$$
\left\|A_{i} \hat{S}_{t}\right\|_{\hat{1} \rightarrow \infty} \leq \varepsilon\left\|H \hat{S}_{t}\right\|_{\hat{1} \rightarrow \infty}+a_{\delta} \varepsilon^{-1-\delta}\left\|\hat{S}_{t}\right\|_{\hat{1} \rightarrow \infty}
$$

and

$$
\left\|A_{i} S_{t}\right\|_{\infty \rightarrow \infty} \leq \varepsilon\left\|H S_{t}\right\|_{\infty \rightarrow \infty}+a_{\delta} \varepsilon^{-1-\delta}\left\|S_{t}\right\|_{\infty \rightarrow \infty}
$$

The first of these bounds together with (10.13) and (10.17) then gives

$$
\left\|A_{i} \hat{S}_{t}\right\|_{\hat{1} \rightarrow \infty} \leq a t^{-D^{\prime} / 2}\left(\varepsilon t^{-1}+a_{\delta} \varepsilon^{-1-\delta}\right) e^{\omega t}
$$

whilst the second with the bounds of Corollary 10.2 gives

$$
\left\|A_{i} S_{t}\right\|_{\infty \rightarrow \infty} \leq a\left(\varepsilon t^{-1}+a_{\delta} \varepsilon^{-1-\delta}\right) e^{\omega t}
$$

for all $t>0$ and $\varepsilon \in\langle 0,1]$. Therefore choosing $\varepsilon=(1 \wedge t)^{1 /(2+\delta)}$ one obtains the following bounds.

Proposition 10.3 There exist $a_{\delta}^{\prime}, a_{\delta}^{\prime \prime}>0$ and $\omega, \omega^{\prime} \geq 0$ such that

$$
\|\| A_{i} K_{t}\left\|_{\infty}=\right\| A_{i} \hat{S}_{t} \|_{\hat{1} \rightarrow \infty} \leq a_{\delta}^{\prime} t^{-D^{\prime} / 2} t^{-(1+\delta) / 2} e^{\omega t}
$$

and

$$
\left\|\mid A_{i} K_{t}\right\|_{\hat{1}}=\left\|A_{i} S_{t}\right\|_{\infty \rightarrow \infty} \leq a_{\delta}^{\prime \prime} t^{-(1+\delta) / 2} e^{\omega^{\prime} t}
$$

for $i \in\left\{1, \ldots, d^{\prime}\right\}$ and for all $\delta, t>0$. The values of $a_{\delta}^{\prime}, a_{\delta}^{\prime \prime}, \omega$ and $\omega^{\prime}$ depend on the coefficients of $H$ through $\lambda_{c}$, and the norm

$$
c \mapsto\||c|\|_{1}=\sum_{i, j=1}^{d^{\prime}}\left\|c_{i j}\right\|_{\infty ; 1}^{\prime}+\sum_{i=1}^{d^{\prime}}\left(\left\|c_{i}\right\|_{\infty}+\left\|c_{i}^{\prime}\right\|_{\infty ; 1}\right)+\left\|c_{0}\right\|_{\infty} .
$$

Remark The dependence of the constants on the derivatives of the coefficients follows because we have used (3.12). Note that if $H$ is in the asymmetric form (1.17) then it can be re-expressed in the form (10.1) with $c_{i}^{\prime}=0$ and the other coefficients $c_{i j}$ etc. are linear combinations of the $c_{\alpha}$ with $|\alpha|=0,1,2$ and the derivatives $A_{i} c_{\alpha}$ with $i=1, \ldots, d^{\prime}$ and $|\alpha|=2$. Therefore the values of the parameters $a_{\delta}^{\prime}, a_{\delta}^{\prime \prime}, \omega$ and $\omega^{\prime}$ depend on the coefficients of $H$ only through $\lambda_{c}$ and the norm $\|c\|_{1}^{\prime}$ defined by (3.4). 
The bounds on the derivatives $A_{i} K_{t}$ can be refined by use of the inequalities (3.37) in place of (3.12) together with some interpolation theory. The starting point is the observation that the interpolation norm $\|\cdot\|_{\infty, \infty, \gamma}^{\prime}$ with $\gamma \in\langle 0,1\rangle$ is equivalent to the Lipschitz norm

$$
\varphi \mapsto\|\varphi\|_{\infty}+\sup _{0<t \leq 1}\left(t^{-\gamma} \omega_{\varphi}(t)\right)
$$

where

$$
\omega_{\varphi}(t)=\sup _{0<|g|^{\prime} \leq t}\|(I-L(g)) \varphi\|_{\infty} .
$$

This is established in [ElR1] Theorem 3.2. Therefore

$$
\|(I-L(g)) \varphi\|_{\infty} \leq c_{\gamma}\left(|g|^{\prime}\right)^{\gamma}\|\varphi\|_{\infty, \infty, \gamma}^{\prime}
$$

for a suitable $c_{\gamma}>0$. But

$$
\left(L_{\hat{p}}, L_{\hat{p} ; 1}^{\prime}\right)_{q, \gamma} \subseteq\left(L_{\hat{p}}, L_{\hat{p} ; 1}^{\prime}\right)_{\infty, \gamma}
$$

and the embedding is continuous (see, for example, [BuB] Corollary 3.2.13). Hence one has bounds

$$
\|(I-L(g)) \varphi\|_{\infty} \leq c_{q, \gamma}\left(|g|^{\prime}\right)^{\gamma}\|\varphi\|_{\infty, q, \gamma}^{\prime}
$$

for all $q \in[1, \infty\rangle$ and a suitable $c_{q, \gamma}>0$. Then by (3.37) one obtains estimates

$$
\begin{aligned}
\left\|(I-L(g)) A_{i} \varphi\right\|_{\infty} & \leq c_{q, \gamma}\left(|g|^{\prime}\right)^{\gamma}\|\varphi\|_{\infty, q, \gamma ; 1}^{\prime} \\
& \leq c_{q, \gamma}\left(|g|^{\prime}\right)^{\gamma}\left(\varepsilon\|H \varphi\|_{\infty, q, \gamma}^{\prime}+a_{\delta}^{\prime} \varepsilon^{-1-\delta}\|\varphi\|_{\infty, q, \gamma}^{\prime}\right)
\end{aligned}
$$

with the value of $a_{\delta}^{\prime}$ depending on the coefficients of $H$ through $\lambda_{c}$ and the norm III $\mid \|_{1}$ of Proposition 10.3.

Next one has bounds

$$
\|\varphi\|_{\infty, q, \gamma}^{\prime} \leq \varepsilon^{1-\gamma}\|\varphi\|_{\infty ; 1}^{\prime}+c_{q, \gamma}^{\prime} \varepsilon^{-\gamma}\|\varphi\|_{\infty}
$$

by (4.4) in Chapter II of [Rob1]. Thus combining this with (3.12) one establishes that

$$
\|\varphi\|_{\infty, q, \gamma}^{\prime} \leq \varepsilon^{2-\gamma}\|H \varphi\|_{\infty}+c_{q, \gamma, \delta} \varepsilon^{-\gamma-\delta}\|\varphi\|_{\infty}
$$

for all $\varepsilon \in\langle 0,1]$, each $\delta>0$ and a suitable $c_{q, \gamma, \delta}>0$. But since the closure of $H$ generates a continuous semigroup one has estimates

$$
\|H \varphi\|_{\infty} \leq \varepsilon^{2}\left\|H^{2} \varphi\right\|_{\infty}+a^{\prime} \varepsilon^{-2}\|\varphi\|_{\infty}
$$


for all $\varphi \in-D\left(H^{2}\right), \varepsilon>0$ and a suitable $a^{\prime}>0$ (see, Lemma II.2.5 of [Rob1]). The value of $a^{\prime}$ can be estimated in terms of the $a$ and $\omega$ of Corollary 10.2 and hence it only depends on the coefficients of $H$ through $\lambda_{c}$ and $\||c|\|$. Combination of these last two sets of estimates now immediately yields bounds

$$
\|\varphi\|_{\infty, q, \gamma}^{\prime} \leq \varepsilon^{4-\gamma}\left\|H^{2} \varphi\right\|_{\infty}+c_{q, \gamma, \delta}^{\prime} \varepsilon^{-\gamma-\delta}\|\varphi\|_{\infty}
$$

for all $\varepsilon \in\langle 0,1]$. Similarly

$$
\begin{aligned}
\|H \varphi\|_{\infty, q, \gamma}^{\prime} & \leq \varepsilon^{2-\gamma}\left\|H^{2} \varphi\right\|_{\infty}+c_{q, \gamma, \delta} \varepsilon^{-\gamma-\delta}\|H \varphi\|_{\infty} \\
& \leq \varepsilon^{2-\gamma-\delta}\left\|H^{2} \varphi\right\|_{\infty}+c_{q, \gamma, \delta}^{\prime \prime} \varepsilon^{-2-\gamma-\delta}\|\varphi\|_{\infty}
\end{aligned}
$$

for all $\varepsilon \in\langle 0,1]$ and $\gamma, \delta \in\langle 0,1\rangle$. Therefore by combination of (10.23)-(10.25) one concludes that there is a $C_{\gamma, \delta}>0$ such that

$$
\left\|(I-L(g)) A_{i} \varphi\right\|_{\infty} \leq C_{\gamma, \delta}\left(|g|^{\prime}\right)^{\gamma}\left(\varepsilon^{3-\gamma-\delta}\left\|H^{2} \varphi\right\|_{\infty}+\varepsilon^{-1-\gamma-2 \delta}\|\varphi\|_{\infty}\right)
$$

for all $\varepsilon \in\langle 0,1]$. Now replacing $\varphi$ by $S_{t} \varphi$ one can combine these bounds with (10.13) and (10.18) or with the bounds of Corollary 10.2 as in the deduction of Proposition 10.3 to obtain improved estimates on the derivatives $A_{i} K_{t}$. If one makes the choice $\varepsilon=(1 \wedge t)^{-2(1+\gamma+2 \delta) /(4+\delta)}$ one obtains the bounds

$$
\begin{aligned}
\|\|(I-L(g)) A_{i} K_{t} \|_{\infty} & =\left\|(I-L(g)) A_{i} \hat{S}_{t}\right\|_{\hat{1} \rightarrow \infty} \\
& \leq a_{\gamma, \delta}\left(|g|^{\prime}\right)^{\gamma} t^{-D^{\prime} / 2} t^{-2(1+\gamma+2 \delta) /(4+\delta)} e^{\omega t}
\end{aligned}
$$

and

$$
\begin{aligned}
\left\|(I-L(g)) A_{i} K_{t}\right\|_{\hat{\mathbf{1}}} & =\left\|(I-L(g)) A_{i} S_{t}\right\|_{\infty \rightarrow \infty} \\
& \leq a_{\gamma, \delta}^{\prime}\left(|g|^{\prime}\right)^{\gamma} t^{-2(1+\gamma+2 \delta) /(4+\delta)} e^{\omega^{\prime} t}
\end{aligned}
$$

for all $g \in G, \delta, \gamma \in\langle 0,1\rangle$ and $t>0$. But since $2(1+\gamma+2 \delta) /(4+\delta)$ can be expressed as $\left(1+\gamma+\delta^{\prime}\right) / 2$ one has the following version of the previous proposition.

Proposition 10.4 There exist $a_{\gamma, \delta}, a_{\gamma, \delta}^{\prime}>0$ and $\omega, \omega^{\prime} \geq 0$ such that

$$
\begin{aligned}
\|\|(I-L(g)) A_{i} K_{t} \|_{\infty} & =\left\|(I-L(g)) A_{i} \hat{S}_{t}\right\|_{\hat{1} \rightarrow \infty} \\
& \leq a_{\gamma, \delta}\left(|g|^{\prime}\right)^{\gamma} t^{-D^{\prime} / 2} t^{-(1+\gamma+\delta) / 2} e^{\omega t}
\end{aligned}
$$


and

$$
\begin{aligned}
\left\|(I-L(g)) A_{i} K_{t}\right\|_{\hat{1}} & =\left\|(I-L(g)) A_{i} S_{t}\right\|_{\infty \rightarrow \infty} \\
& \leq a_{\gamma, \delta}^{\prime}\left(|g|^{\prime}\right)^{\gamma} t^{-(1+\gamma+\delta) / 2} e^{\omega^{\prime} t}
\end{aligned}
$$

for all $g \in G, \delta, \gamma \in\langle 0,1\rangle$ and $t>0$. The values of $a_{\gamma, \delta}, a_{\gamma, \delta}^{\prime}, \omega$ and $\omega^{\prime}$ depend on the coefficients of $H$ through $\lambda_{c}$, and the norm

$$
c \mapsto\|c \mid\|_{1}=\sum_{i, j=1}^{d^{\prime}}\left\|c_{i j}\right\|_{\infty ; 1}^{\prime}+\sum_{i=1}^{d^{\prime}}\left(\left\|c_{i}\right\|_{\infty}+\left\|c_{i}^{\prime}\right\|_{\infty ; 1}\right)+\left\|c_{0}\right\|_{\infty} .
$$

Note that for each $\gamma \in\langle 0,1\rangle$ the proposition gives estimates

$$
\left\|(I-L(g)) A_{i} S_{t}\right\|_{\infty \rightarrow \infty} \leq a\left(|g|^{\prime}\right)^{\gamma} t^{-(1-\varepsilon)}
$$

for all $g \in G, t \in\langle 0,1]$ and any $\varepsilon \in\langle 0,1-\gamma\rangle$ and the value of $a$ depends on the coefficients of $H$ through $\lambda_{c}$ and $\||c|\|_{1}$. Hence if $H$ is in the asymmetric form (1.17) these estimates hold with $a$ dependent on the coefficients only through $\lambda_{c}$ and the norm $\|c\|_{1}^{\prime}$ defined by (3.4).

We conclude this section with some remarks on the connection between the $L_{\infty}$ conjecture of Section 1 and the derivative bounds on the semigroup kernel.

If the $L_{\infty}$-conjecture is valid then the bounds of Proposition 10.3 hold in the limiting case $\delta=0$ by an identical argument. Conversely, if the bounds

$$
\left\|A_{i} K_{t}\right\|_{\hat{1}}=\left\|A_{i} S_{t}\right\|_{\infty \rightarrow \infty} \leq a t^{-1 / 2} e^{\omega t}
$$

are valid for all $t>0$, with $a$ and $\omega$ dependent on the coefficients of $H$ only through $\lambda_{c}$ and $\|c\|_{1}^{\prime}$ then the $L_{\infty}$-conjecture is also verified. This follows because

$$
\begin{aligned}
\left\|A_{i}(\lambda I+H)^{-1}\right\|_{\infty \rightarrow \infty} & \leq \int_{0}^{\infty} d t e^{-\lambda t}\left\|A_{i} S_{t}\right\|_{\infty \rightarrow \infty} \\
& =\int_{0}^{\infty} d t e^{-\lambda t}\left\|A_{i} K_{t}\right\|_{\hat{1}} \\
& \leq \int_{0}^{\infty} d t t^{-1 / 2} e^{-(\lambda-\omega) t} \leq a^{\prime}(\lambda-\omega)^{-1 / 2}
\end{aligned}
$$

for $\lambda>\omega$. Therefore

$$
\left\|A_{i} \varphi\right\|_{\infty} \leq a^{\prime}(\lambda-\omega)^{-1 / 2}\left(\|H \varphi\|_{\infty}+\lambda\|\varphi\|_{\infty}\right)
$$

for all $\lambda>\omega$ and this immediately verifies the $L_{\infty}$-conjecture. Consequently one concludes that the $L_{\infty}$-conjecture is equivalent to the bounds (10.30). 
If one eonsiders special classes of subelliptic operators or special classes of groups the foregoing upper bounds can be improved in several ways. The improvements are described in [Rob2] for subelliptic operators with constant coefficients and strongly elliptic operators with variable coefficients. Most of the arguments used in these special cases extend directly to the context of subelliptic operators with variable coefficients. We mention some of these conclusions without proof.

Suppose

$$
H=-\sum_{i, j=1}^{d^{\prime}} A_{i} c_{i j} A_{i}
$$

is a pure second-order subelliptic operator with $c_{i j} \in L_{\infty ; 5}$ and suppose further that $G$ is a unimodular group. If $D$ is chosen such that

$$
\left|B_{r}\right| \geq k r^{D}
$$

for some $k>0$ and all $r \geq 1$ then the semigroup kernel $K$ associated with $H$ satisfies bounds

$$
\begin{aligned}
0 & \leq K_{t}(g ; h) \leq a\left(1 \wedge \varepsilon \lambda_{c} t\right)^{-D^{\prime} / 2}\left(1 \vee \varepsilon \lambda_{c} t\right)^{-D / 2} e^{-\left(\left|g h^{-1}\right|^{\prime}\right)^{2} / 4 \Lambda_{c}(1+\varepsilon) t} \\
\left|\partial_{t}^{n} K_{t}(g ; h)\right| & \leq a b^{n} n !\left(\varepsilon^{2} t\right)^{-n}\left(1 \wedge \varepsilon \lambda_{c} t\right)^{-D^{\prime} / 2}\left(1 \vee \varepsilon \lambda_{c} t\right)^{-D / 2} e^{-\left(\left|g h^{-1}\right|^{\prime}\right)^{2} / 4 \Lambda_{c}(1+\varepsilon) t}
\end{aligned}
$$

for all $g, h \in G, t>0$, and $\varepsilon \in\langle 0,1]$. The value of the positive parameter $a$ is independent of the coefficients of $H$.

If $G$ is a polynomial group then one can chose $D$ such that $\left|B_{r}^{\prime}\right|=O\left(r^{D}\right)$ as $r \rightarrow \infty$ and since $\left|B_{r}^{\prime}\right|=\mathrm{O}\left(r^{D^{\prime}}\right)$ as $r \rightarrow 0$ the bounds (10.33) and (10.34) can be re-expressed in the form

$$
\begin{aligned}
0 \leq K_{t}(g ; h) & \leq a\left|B_{\varepsilon \lambda_{c} t}^{\prime}\right|^{-1 / 2} e^{-\left(\left|g h^{-1}\right|^{\prime}\right)^{2} / 4 \Lambda_{c}(1+\varepsilon) t} \\
\left|\left(\partial_{t}^{n} K_{t}\right)(g ; h)\right| & \leq a b^{n} n !\left(\varepsilon^{2} t\right)^{-n}\left|B_{\varepsilon \lambda_{c} t}^{\prime}\right|^{-1 / 2} e^{-\left(\left|g h^{-1}\right|^{\prime}\right)^{2} / 4 \Lambda_{c}(1+\varepsilon) t} .
\end{aligned}
$$

All estimates on the derivatives $A_{i} K_{t}$ depend, however, on some version of the a priori inequalities (3.12). Any essential improvement to these bounds would require extending the inequalities to the case $\delta=0$ under minimal smoothness hypotheses on the coefficients of $H$.

Finally Corollary 10.2 established that the subelliptic semigroups $S$ are holomorphic in an open sector $\Delta_{\delta}$ with $\delta$ determined by the parameters $\lambda_{c}$, and $\||c|\|$, i.e., the 
sector is independent of the derivatives of the coefficients. Then considering the semigroup $S_{e^{i \theta} t}$ with $|\theta|<\delta$ one can bound the corresponding kernel $K_{e^{i \theta t} t}$ by the foregoing techniques for all small $\theta$. One obtains bounds with the same general characteristics which again do not depend upon the derivatives of the coefficients. 


\section{Generator Theorems}

In this section we prove the generator statements of Theorem 1.1 by using approximation arguments to exploit the results already obtained for smooth coefficients. We begin by considering a sequence of symmetric second-order operators

$$
H_{n}=-\sum_{i, j=1}^{d^{\prime}} A_{i} c_{i j}^{(n)} A_{j}+c_{0}
$$

with coefficients $c_{i j}^{(n)} \in L_{\infty ; 5}$ and $c_{0} \in L_{\infty}$. Then by Proposition 8.2 the closures of the $H_{n}$ generate consistent interpolating semigroups $S^{(n)}$. Next we consider convergence of the $S^{(n)}$ and the corresponding kernels $K^{(n)}$ for sequences such that

$$
\lim _{n, m \rightarrow \infty} \sum_{i, j=1}^{d^{\prime}}\left\|c_{i j}^{(n)}-c_{i j}^{(m)}\right\|_{\infty}=0
$$

We assume that the ellipticity constants of the $\left(c_{i j}^{(n)}\right)$ are bounded away from zero uniformly, i.e., there is a $\lambda_{c}>0$ such that $\left(c_{i j}^{(n)}(g)\right) \geq \lambda_{c} I$ for all $g \in G$ and $n=$ $1,2, \ldots$. Moreover, we assume that the sequence

$$
n \mapsto \sum_{i, j=1}^{d^{\prime}}\left\|c_{i j}^{(n)}\right\|_{\infty ; 1}^{\prime}+\left\|c_{0}\right\|_{\infty}
$$

has a finite upper bound which we denote by $C_{1}$.

First, if the $S^{(n)}$ act on $L_{\hat{2}}$ then

$$
\left\langle\varphi,\left(S_{t}^{(n)}-S_{t}^{(m)}\right) \psi\right\rangle=t \int_{0}^{1} d \lambda \sum_{i, j=1}^{d^{\prime}}\left\langle\left(A_{i}-\beta_{i} I\right) \tilde{S}_{\lambda t}^{(n)} \varphi,\left(c_{i j}^{(n)}-c_{i j}^{(m)}\right) A_{j} S_{(1-\lambda) t}^{(m)} \psi\right\rangle
$$

for all $\varphi, \psi \in L_{\hat{2}}$ where $\tilde{S}^{(n)}$ denotes the adjoint of $S^{(n)}$ with respect to right Haar measure, i.e., the semigroup generated by the closure of the operator obtained from $H_{n}$ by the replacement $A_{i} \rightarrow-A_{i}+\beta_{i} I$. Therefore

$$
\left|\left\langle\varphi,\left(S_{t}^{(n)}-S_{t}^{(m)}\right) \psi\right\rangle\right| \leq t \int_{0}^{1} d \lambda\left\|\tilde{S}_{\lambda t}^{(n)} \varphi\right\|_{\hat{2} ; 1}^{\prime} N_{\hat{2} ; 1}^{\prime}\left(S_{(1-\lambda) t}^{(m)} \psi\right)\left\|c^{(n)}-c^{(m)}\right\|_{\infty}
$$


where

$$
\left\|c^{(n)}-c^{(m)}\right\|_{\infty}=\sum_{i, j=1}^{d^{\prime}}\left\|c_{i j}^{(n)}-c_{i j}^{(m)}\right\|_{\infty} .
$$

But it follows from the discussion preceding Proposition 3.1 and the estimates of Corollary 10.2 that one has uniform bounds

$$
\begin{aligned}
\left\|\tilde{S}_{\lambda t}^{(n)} \varphi\right\|_{\hat{2} ; 1}^{\prime} & \leq a(\lambda t)^{-1 / 2} e^{\omega t}\|\varphi\|_{\hat{2}} \\
N_{\hat{2} ; 1}^{\prime}\left(S_{(1-\lambda) t}^{(m)} \psi\right) & \leq a((1-\lambda) t)^{-1 / 2} e^{\omega(1-\lambda) t}\|\psi\|_{\hat{2}}
\end{aligned}
$$

where the values of $a$ and $\omega$ depend only on the lower bound $\lambda_{c}$ of the ellipticity constants and the upper bound of the norms $\left\|c_{i j}^{(n)}\right\|_{\infty}$. Therefore

$$
\left\|S_{t}^{(n)}=S_{t}^{(m)}\right\|_{\hat{2} \rightarrow \hat{2}} \leq a^{2}\left\|c^{(n)}-c^{(m)}\right\|_{\infty} e^{\omega t} \int_{0}^{1} d \lambda \lambda^{-1 / 2}(1-\lambda)^{-1 / 2}
$$

Consequently

$$
\lim _{n, m \rightarrow \infty}\left\|S_{t}^{(n)}-S_{t}^{(m)}\right\|_{\hat{2} \rightarrow \hat{2}}=0
$$

uniformly for $t \in[0,1]$. A similar but simpler calculation establishes that

$$
\lim _{n, m \rightarrow \infty}\left\|S_{t}^{(n)}-S_{t}^{(m)}\right\|_{2 \rightarrow 2}=0
$$

where the convergence is again uniform $t \in[0,1]$. We will use both these results but first we exploit the $L_{\hat{2}}$-convergence. In fact for the subsequent purposes it suffices to suppose that the semigroups are strongly convergent on $L_{\hat{2}}$ and this can be expressed in terms of the semigroup kernel as

$$
\lim _{n, m \rightarrow \infty} \int_{G} d \hat{g}\left|\int_{G} d \hat{h}\left(K_{t}^{(n)}(g ; h)-K_{t}^{(m)}(g ; h)\right) \varphi(h)\right|^{2}=0
$$

for $t \in\langle 0,1]$ and $\varphi \in L_{\overline{2}}$.

Next, remark that Proposition 10.3 gives bounds

$$
\begin{aligned}
\left|\left(A_{i} K_{t}^{(n)}\right)(g ; h)\right| & \leq a_{\delta} t^{-D^{\prime} / 2} t^{-(1+\delta) / 2} e^{\omega^{\prime} t} \\
\int_{G} d \hat{h}\left|\left(A_{i} K_{t}^{(n)}\right)(g ; h)\right| & \leq a_{\delta} t^{-(1+\delta) / 2} e^{\omega^{\prime} t}
\end{aligned}
$$

uniform both in $n$ and $g, h \in G$, or $g \in G$, where the values of $a_{\delta}$ and $\omega^{\prime}$ depend on $\lambda_{c}$ and $C_{1}$. Therefore one has $L_{\hat{2}}$-estimates

$$
\int_{G} d \hat{h}\left|\left(A_{i} K_{t}^{(n)}\right)(g ; h)\right|^{2} \leq a_{\delta}^{2} t^{-D^{\prime} / 2} t^{-(1+\delta)} e^{2 \omega^{\prime} t}
$$


uniform for all $g \in G$. But (11.2) and (11.5) together imply

$$
\lim _{n, m \rightarrow \infty} \operatorname{ess} \sup _{g \in G} \int_{G} d \hat{h}\left|K_{t}^{(n)}(g ; h)-K_{t}^{(m)}(g ; h)\right|^{2}=0
$$

This is equivalent to the convergence

$$
\lim _{n, m \rightarrow \infty}\left\|\mid K_{t}^{(n)}-K_{t}^{(m)}\right\|_{\hat{2}}=0
$$

and is established by the following reasoning.

If (11.6) is false then there is an $\varepsilon>0$, a $g_{0} \in G$, a $\varphi \in L_{\hat{2}}$ with $\|\varphi\|_{\hat{2}}=1$, and arbitrary large $n, m$ such that

$$
\left|\int_{G} d \hat{h}\left(K_{t}^{(n)}\left(g_{0} ; h\right)-K_{t}^{(m)}\left(g_{0} ; h\right)\right) \varphi(h)\right|>\varepsilon .
$$

But if $\gamma$ is a suitable piecewise differentiable path from $g_{0}$ to $g$ then

$$
\begin{aligned}
\left|K_{t}^{(n)}(g ; h)-K_{t}^{(n)}\left(g_{0} ; h\right)\right| & =\left|\int_{0}^{1} d s \sum_{i=1}^{d^{\prime}} \gamma_{i}(s)\left(A_{i} K_{t}^{(n)}\right)(\gamma(s) ; h)\right| \\
& \leq \int_{0}^{1} d s\left(\sum_{i=1}^{d^{\prime}} \gamma_{i}(s)^{2}\right)^{1 / 2}\left(\sum_{i=1}^{d^{\prime}}\left|\left(A_{i} K_{t}^{(n)}\right)(\gamma(s) ; h)\right|^{2}\right)^{1 / 2}
\end{aligned}
$$

where $\gamma_{i}(s)$ is defined by (1.11). Therefore, by the Minkowski inequality,

$$
\begin{aligned}
&\left(\int_{G} d \hat{h}\left|K_{t}^{(n)}(g ; h)-K_{t}^{(n)}\left(g_{0} ; h\right)\right|^{2}\right)^{1 / 2} \leq \int_{0}^{1} d s\left(\sum_{i=1}^{d^{\prime}} \gamma_{i}(s)^{2}\right)^{1 / 2} \cdot \\
& \cdot\left(\int_{G} d \hat{h} \sum_{i=1}^{d^{\prime}}\left|\left(A_{i} K_{t}^{(n)}\right)(\gamma(s) ; h)\right|^{2}\right)^{1 / 2}
\end{aligned}
$$

Hence it follows from the definition (1.13) of the modulus $|\cdot|^{\prime}$ and the estimates (11.5) that for each $t>0$ there is a $C_{t}>0$, proportional to $t^{-D^{\prime} / 4} t^{-(1+\delta) / 2} e^{\omega^{\prime} t}$, such that

$$
\left(\int_{G} d \hat{h}\left|K_{t}^{(n)}(g ; h)-K_{t}^{(n)}\left(g_{0} ; h\right)\right|^{2}\right)^{1 / 2} \leq\left|g g_{0}^{-1}\right|^{\prime} C_{t}
$$

Consequently, if $\Omega_{t}$ is the ball

$$
\Omega_{t}=\left\{g ;\left|g g_{0}^{-1}\right|^{\prime}<\varepsilon / 3 C_{t}\right\}
$$

one deduces from (11.7) that

$$
\left|\int_{G} d \hat{h}\left(K_{t}^{(n)}(g ; h)-K_{t}^{(m)}(g ; h)\right) \varphi(h)\right| \geq \varepsilon / 3
$$


for all $g \in \Omega_{t}$. Since this contradicts (11.2) one concludes that (11.6) is valid.

Now we argue that one also has the convergence

$$
\lim _{n, m \rightarrow \infty} \underset{g \in G}{\operatorname{ess} \sup _{G}} \int_{G} d \hat{h}\left|K_{t}^{(n)}(g ; h)-K_{t}^{(m)}(g ; h)\right|=0
$$

or, equivalently,

$$
\lim _{n, m \rightarrow \infty}\left\|K_{t}^{(n)}-K_{t}^{(m)}\right\|_{\hat{\mathbf{1}}}=0 .
$$

To this end we observe that if $B_{r}^{\prime}(g)=\left\{h ;\left|g h^{-1}\right|^{\prime}<r\right\}$ then

$$
\int_{G \backslash B_{r}^{\prime}(g)} d \hat{h} K_{t}^{(n)}(g ; h) \leq a e^{-b r^{2} / t} e^{\omega t}
$$

with $a, b, \omega>0$ uniformly in $g$ and $n$ as a direct corollary of the Gaussian estimates of Proposition 10.1. Moreover

$$
\begin{aligned}
\left|B_{r}^{\prime}(g)\right| & =\int d \hat{h}\left\{h ;\left|g h^{-1}\right|^{\prime}<r\right\} \\
& =\int d \hat{h}\left\{h ;\left|h g^{-1}\right|^{\prime}<r\right\}=\left|B_{r}^{\prime}(e)\right|
\end{aligned}
$$

by right invariance of the Haar measure. Hence

$$
\int_{G} d \hat{h}\left|K_{t}^{(n)}(g ; h)-K_{t}^{(m)}(g ; h)\right| \leq\left|B_{r}^{\prime}(e)\right|^{1 / 2} \cdot|| \mid K_{t}^{(n)}-K_{t}^{(m)} \|_{\hat{2}}+2 a e^{-b r^{2} / t} e^{\omega t} .
$$

Here we have separated the integral into a part over $B_{r}^{\prime}(g)$ and a remainder and then used the Schwarz inequality on the first part. Now (11.8) follows immediately.

The important feature of these estimates are that (11.9) is equivalent to

$$
\lim _{n, m \rightarrow \infty}\left\|S_{t}^{(n)}-S_{t}^{(m)}\right\|_{\infty \rightarrow \infty}=0
$$

It is this observation that is the key to establishing the first generator result for operators with coefficients which are not smooth.

Proposition 11.1 Let $H$ be a subelliptic operator in the form

$$
H=-\sum_{i, j=1}^{d^{\prime}} A_{i} c_{i j} A_{i}+\sum_{i=1}^{d^{\prime}} c_{i} A_{i}+c_{0}
$$

with coefficients $c_{i j} \in L_{\infty ; 1}^{\prime}$ and $c_{i}, c_{0} \in L_{\infty}$.

Then $H$ has a closed extension on $L_{\infty}$ which generates a weakly* continuous holomorphic semigroup $T$. 
Proof First we consider the case $c_{i}=0$. Let $\varphi_{n} \in C_{c}^{\infty}$ be positive functions normalized with respect to left Haar measure and define $c_{i j}^{(n)}$ by right regularization of $c_{i j}$ with $\varphi_{n}$, i.e.,

$$
c_{i j}^{(n)}(g)=\int_{G} d h \varphi_{n}(h) c_{i j}(g h)=\left(R\left(\varphi_{n}\right) c_{i j}\right)(g)
$$

where $R$ denotes right translations. Then

$$
\left\|c_{i j}^{(n)}\right\|_{\infty ; 1}^{\prime} \leq\left\|c_{i j}\right\|_{\infty ; 1}^{\prime}
$$

and

$$
\left(c_{i j}^{(n)}(g)\right) \geq \lambda_{c} I
$$

where $\lambda_{c}$ is the ellipticity constant of $H$. But as $c_{i j} \in L_{\infty ; 1}^{\prime}$ the coefficients are uniformly continuous by (1.16) and hence one can choose the $\varphi_{n}$ such that

$$
\lim _{n \rightarrow \infty}\left\|c_{i j}^{(n)}-c_{i j}\right\|_{\infty}=\lim _{n \rightarrow \infty} \int_{G} d h \varphi_{n}(h)\left\|(I-R(h)) c_{i j}\right\|_{\infty}=0
$$

Moreover, as $\varphi_{n} \in C_{c}^{\infty}$ it follows that $c_{i j}^{(n)} \in L_{\infty ; \infty}$.

Next remark that

$$
\lim _{n \rightarrow \infty}\left(\psi,\left(A_{k} c_{i j}^{(n)}\right) \chi\right)=\left(\psi,\left(A_{k} c_{i j}\right) \chi\right)
$$

for all $\psi \in L_{1}$ and $\chi \in L_{\infty}$ by the following reasoning. First $A_{k} c_{i j}^{(n)}=\left(A_{k} c_{i j}\right)^{(n)}$ because $A_{k}$ is a left derivative and the regularization is over right translations. Secondly, if $b=A_{k} c_{i j}$ then

$$
\left(\psi,\left(b^{(n)}-b\right) \chi\right)=\int_{G} d h \varphi_{n}(h) \int_{G} d g \psi(g)(b(g h)-b(g)) \chi(g)
$$

But

$$
(b(g h)-b(g)) \psi(g)=b(g h)(\psi(g)-\psi(g h))-(b(g) \psi(g)-b(g h) \psi(g h))
$$

Thus

$\left|\left(\psi,\left(b^{(n)}-b\right) \chi\right)\right| \leq \int_{G} d h \varphi_{n}(h)\left\{\|b\|_{\infty}\|(I-R(h)) \psi\|_{1}+\|(I-R(h)) b \psi\|_{1}\right\}\|\chi\|_{\infty}$.

Since right translations are strongly continuous on $L_{1}$ it follows from these bounds that $\left(\psi,\left(b^{(n)}-b\right) \chi\right) \rightarrow 0$ as $n \rightarrow \infty$. 
Now let $H_{n}$ denote the subelliptic operators constructed with the coefficients $c_{i j}^{(n)}$ and $c_{0}$ and $S^{(n)}$ the corresponding semigroups. Then the $S^{(n)}$ are uniformly convergent on $L_{\infty}$ by (11.10) and the limits automatically define a weakly* continuous semigroup $S$ on $L_{\infty}$. Moreover, since the semigroup kernels $K^{(n)}$ associated with the $S^{(n)}$ converge with respect to the $\|\mid \cdot\| \|_{\hat{1}}$-norm, by (11.9), it follows that the limit $K$ is the kernel of $S$.

Next we argue that the generator $H_{0}$ of $S$ is an extension of $H$.

If $\varphi \in C_{c}^{\infty}$ and $\psi \in L_{1} \cap L_{2}$ then approximating $S$ and $H$ by $S^{(n)}$ and $H_{n}$, respectively, one has

$$
\begin{aligned}
t^{-1}\left|\left(\psi,\left(I-S_{t}\right) \varphi\right)-\int_{0}^{t} d s\left(\psi, S_{s} H \varphi\right)\right| \leq t^{-1}\|\psi\|_{2}\|\varphi\|_{2}\left\|S_{t}-S_{t}^{(n)}\right\|_{2 \rightarrow 2} \\
-\quad+C_{1}\|\psi\|_{2}\|\varphi\|_{2 ; 2}^{\prime} \sup _{0 \leq s \leq 1}\left\|S_{s}-S_{s}^{(n)}\right\|_{2 \rightarrow 2}+t^{-1} \int_{0}^{t} d s\left|\left(\psi, S_{s}\left(H-H_{n}\right) \varphi\right)\right|
\end{aligned}
$$

Hence taking the limit $n \rightarrow \infty$ and using the uniformity of the convergence of $S^{(n)}$ to $S$ on $L_{2}$ one obtains the estimates

$$
t^{-1}\left|\left(\psi,\left(I-S_{t}\right) \varphi\right)-\int_{0}^{t} d s\left(\psi, S_{s} H \varphi\right)\right| \leq \limsup _{n \rightarrow \infty} t^{-1} \int_{0}^{t} d s\left|\left(\psi, S_{s}\left(H-H_{n}\right) \varphi\right)\right| .
$$

Now the integrand on the right hand side is a sum of terms of the form $\left|\left(\psi, S_{s} \varphi_{n}\right)\right|$ with the $\varphi_{n}$ a sequence of $L_{\infty}$-functions which is weak* convergent to zero, by the argument of the previous paragraph, and such that the sequence of norms $\left\|\varphi_{n}\right\|_{\infty}$ has a uniform bound $C_{\varphi}$. But as $S$ is continuous one has bounds $\left\|S_{s}\right\|_{\infty \rightarrow \infty} \leq M e^{\omega s}$ and consequently

$$
\left|\left(\psi, S_{s} \varphi_{n}\right)\right| \leq M e^{\omega s} C_{\varphi}\|\psi\|_{1}
$$

Thus

$$
\limsup _{n \rightarrow \infty} t^{-1} \int_{0}^{t} d s\left|\left(\psi, S_{s}\left(H-H_{n}\right) \varphi\right)\right|=0
$$

by the Lebesgue dominated convergence theorem. Consequently,

$$
t^{-1}\left(\psi,\left(I-S_{t}\right) \varphi\right)=t^{-1} \int_{0}^{t} d s\left(\psi, S_{s} H \varphi\right)
$$

for all $\varphi \in C_{c}^{\infty}$ and $\psi \in L_{1} \cap L_{2}$. But then the relation extends to all $\psi \in L_{1}$ by continuity. Finally since $C_{c}^{\infty}$ is a core for $\bar{H}$ in the weak ${ }^{*}$ topology it follows that $H_{0}$ is an extension of $H$. 
Now we consider holomorphy properties. The approximating sequence of semigroups are all holomorphic, by Proposition 9.5 and it follows from Corollary 10.2 that one has bounds

$$
\left\|H_{n} S_{t}^{(n)}\right\|_{\infty \rightarrow \infty} \leq a t^{-1} e^{\omega t}
$$

uniform in $n$ with the values of $a$ and $\omega$ determined by $\lambda_{c}$ and $C_{1}$. Thus

$$
\begin{aligned}
s^{-1}\left\|\left(I-S_{s}\right) S_{t}\right\|_{\infty \rightarrow \infty} & =\lim _{n \rightarrow \infty} s^{-1}\left\|S_{s}^{(n)}-S_{t+s}^{(n)}\right\|_{\infty \rightarrow \infty} \\
& \leq s^{-1} \int_{t}^{s+t} d u\left\|H_{n} S_{u}^{(n)}\right\|_{\infty \rightarrow \infty} \\
& \leq a^{\prime} t^{-1} e^{\omega^{\prime} t}
\end{aligned}
$$

for all $s \in\left\langle 0^{-}, 1\right]$ with the values of $a^{\prime}$ and $\omega^{\prime}$ again determined by $\lambda_{c}$ and $C_{1}$. But then it follows (see, for example, [BrR2] Proposition 3.1.23) that $S_{t} L_{\infty} \subseteq D\left(H_{0}\right)$ for $t>0$ and

$$
\left\|H_{0} S_{t}\right\|_{\infty \rightarrow \infty} \leq a^{\prime} t^{-1} e^{\omega^{\prime} t}
$$

Therefore $S$ is a holomorphic semigroup.

Next we deal with the first-order terms by perturbation theory. But this requires a little care because we are considering weakly* continuous semigroups. We begin by establishing that $S_{t} L_{\infty} \subseteq L_{\infty ; 1}^{\prime}$ for $t>0$. To this end note that

$$
s^{-1}\left\|\left(I-L\left(e^{-s a_{i}}\right)\right) S_{t} \varphi\right\|_{\infty} \leq s^{-1}\left\|\left(I-L\left(e^{-s a_{i}}\right)\right) S_{t}^{(n)} \varphi\right\|_{\infty}+2 s^{-1}\left\|\left(S_{t}-S_{t}^{(n)}\right) \varphi\right\|_{\infty} \quad \text {. }
$$

But

$$
\begin{aligned}
s^{-1}\left\|\left(I-L\left(e^{-s a_{i}}\right)\right) S_{t}^{(n)} \varphi\right\|_{\infty} & \leq \underset{g \in G}{\operatorname{ess} \sup _{G}} \int_{G} d \hat{h}\left|\left(A_{i} K_{t}^{(n)}\right)(g ; h)\right| \cdot|\varphi(h)| \\
& \leq\|\varphi\|_{\infty} a_{\delta} t^{-(1+\delta) / 2} e^{\omega^{\prime} t}
\end{aligned}
$$

for $i=1, \ldots, d^{\prime}$ by (11.4). Therefore, using (11.10), one concludes that in the limit $n \rightarrow \infty$

$$
s^{-1}\left\|\left(I-L\left(e^{-s a_{i}}\right)\right) S_{t} \varphi\right\|_{\infty} \leq\|\varphi\|_{\infty} a_{\delta} t^{-(1+\delta) / 2} e^{\omega^{\prime} t}
$$

and since the right hand side is independent of $s$ one deduces that $S_{t} L_{\infty} \subseteq L_{\infty ; 1}^{\prime}$ and

$$
\left\|A_{i} S_{t}\right\|_{\infty \rightarrow \infty} \leq a_{\delta} t^{-(1+\delta) / 2} e^{\omega^{\prime} t}
$$


for all $t>0$. Alternatively, by Laplace transformation, one has

$$
\left\|A_{i}\left(\lambda I+H_{0}\right)^{-1}\right\|_{\infty \rightarrow \infty} \leq a_{\delta}^{\prime} \lambda^{-(1-\delta) / 2}
$$

for all large $\lambda$ where $a_{\delta}^{\prime}$ depends on $a_{\delta}, \omega^{\prime}$ and $\delta$. Hence $A_{i}$ is a small perturbation of $H_{0}$. In particular $D\left(H_{0}\right) \subseteq L_{\infty ; 1}^{\prime}$. Thus if

$$
H_{1}=\sum_{i=1}^{d^{\prime}} c_{i} A_{i}
$$

then $D\left(H_{0}\right) \subseteq D\left(H_{1}\right)$. This together with the small $t$ bounds on $\left\|A_{i} S_{t}\right\|_{\infty \rightarrow \infty}$ suffice to prove that the perturbation series

$$
T_{t}=\sum_{n \geq 1} T_{t}^{(n)}
$$

defined by $T_{t}^{(0)}=S_{t}$ and

$$
T_{t}^{(n)}=-\int_{0}^{t} d s T_{t-s}^{(0)} H_{1} T_{s}^{(n-1)}
$$

is norm-convergent on $L_{\infty}$ for all $t \geq 0$ by the following reasoning.

Let

$$
a_{n}(t)=\left\|T_{t}^{(n)}\right\|_{\infty \rightarrow \infty} \quad, \quad b_{n}(t)=\sup _{1 \leq i \leq d^{\prime}}\left\|A_{i} T_{t}^{(n)}\right\|_{\infty \rightarrow \infty}
$$

and then one has the coupled differential inequalities

$$
\begin{aligned}
& a_{n}(t) \leq c \int_{0}^{t} d s a_{0}(t-s) b_{n-1}(s) \\
& b_{n}(t) \leq c \int_{0}^{t} d s b_{0}(t-s) b_{n-1}(s)
\end{aligned}
$$

with $c=\sum_{i=1}^{d^{\prime}}\left\|c_{i}\right\|_{\infty}$. But one also has bounds

$$
a_{0}(t)=\left\|S_{t}\right\|_{\infty \rightarrow \infty} \leq e^{\omega t} \quad, \quad b_{0}(t)=\sup _{1 \leq i \leq d^{\prime}}\left\|A_{i} S_{t}\right\|_{\infty \rightarrow \infty} \leq a_{\delta} t^{-(1+\delta) / 2} e^{\omega^{\prime} t}
$$

for $\delta \in\langle 0,1]$ and all $t>0$. The first bound is valid with $\omega=\left\|c_{0}\right\|_{\infty}$ and the second follows from (11.11). Now (11.12)-(11.14) are solved by the proposition in the appendix to give bounds

$$
\begin{aligned}
\left\|T_{t}^{(n)}\right\|_{\infty \rightarrow \infty} & \leq a b^{n}\left(t^{n} / n !\right)^{(1-\delta) / 2} e^{\omega t} \\
\sup _{1 \leq i \leq d^{\prime}}\left\|A_{i} T_{t}^{(n)}\right\|_{\infty \rightarrow \infty} & \leq a b^{n}\left(t^{n} / n !\right)^{(1-\delta) / 2} t^{(1+\delta) / 2} e^{\omega t} .
\end{aligned}
$$


These suffice to show that the series

$$
T_{t}=\sum_{n \geq 1} T_{t}^{(n)} \quad, \quad A_{i} T_{t}=\sum_{n \geq 1} A_{i} T_{t}^{(n)}
$$

are norm convergent for all $t \geq 0$ and that one has estimates

$$
\left\|T_{t}\right\|_{\infty \rightarrow \infty} \leq a e^{\omega t},\left\|A_{i} T_{t}\right\|_{\infty \rightarrow \infty} \leq a_{\delta} t^{-(1+\delta) / 2} e^{\omega^{\prime} t}
$$

analogous to (11.14).

The $T_{t}, t \geq 0$, define a weakly* continuous semigroup $T$ on $L_{\infty}$ and it remains to identify its generator. First, it follows from (11.15) that $t^{-1}\left\|T_{t}^{(n)}\right\|_{\infty \rightarrow \infty} \rightarrow 0$ as $t \rightarrow 0$ for $n \geq 2$. Secondly, for $n=1, \psi \in L_{1}$ and $\varphi \in D\left(H_{0}\right)$ one has

$$
\begin{gathered}
\left|\left(\psi,\left(t^{-1} T_{t}^{(1)}-H_{1}\right) \varphi\right)\right| \leq t^{-1} \int_{0}^{t} d s\left|\left(\psi,\left(S_{t-s}-I\right) H_{1} \varphi\right)\right|+ \\
t^{-1} \int_{0}^{t} d s\left|\left(\psi, S_{t-s} H_{1}\left(S_{s}-I\right) \varphi\right)\right| .
\end{gathered}
$$

Now the first term on the right tends to zero because $S$ is weakly* continuous and the second tends to zero by a Duhamel estimate using the estimates (11.14). Therefore one concludes that the generator $\widetilde{H}$ of $T$ extends $H_{0}+H_{1}$. Finally since the bounds (11.17) are valid one can repeat this perturbation construction with $S$ replaced by $T$ and $H_{1}$ by $-H_{1}$ to obtain a semigroup whose generator extends $\widetilde{H}-H_{1}$, which in turn extends $H_{0}$. But a generator cannot have a strict generator extension. Therefore $\widetilde{H}-H_{1}=H_{0}$ or $\widetilde{H}=H_{0}+H_{1}$.

Note that the semigroup $T$ is holomorphic in the same sector as $S$ by the perturbation theory of holomorphic semigroups.

Next we examine regularity properties of the semigroup $T$.

Proposition 11.2 Let $H$ be a subelliptic operator in the form

$$
H=-\sum_{i, j=1}^{d^{\prime}} A_{i} c_{i j} A_{i}+\sum_{i=1}^{d^{\prime}} c_{i} A_{i}+c_{0}
$$

with coefficients $c_{i j} \in L_{\infty ; 1}^{\prime}$ and $c_{i}, c_{0} \in L_{\infty}$ and let $T$ denote the corresponding semigroup on $L_{\infty}$.

Then $T_{t} C_{0} \subseteq C_{0 ; 1}^{\prime}$ for $t>0$ and the restriction of $T$ to $C_{0}$ is strongly continuous. Moreover, for each $\gamma, \varepsilon \in\langle 0,1\rangle$ with $\gamma+\varepsilon<1$ there exists an a $>0$ such that

$$
\left\|(I-L(g)) A_{i} T_{t}\right\|_{\infty \rightarrow \infty} \leq a t^{-(1-\varepsilon)}\left(|g|^{\prime}\right)^{\gamma}
$$

for all $g \in G, t \in\langle 0,1]$ and $i \in\left\{1, \ldots, d^{\prime}\right\}$. 
Proof We begin by establishing the result for the semigroup $S$ generated by the operator with no first-order terms.

It follows from the above construction of $S$ that its action is determined by a kernel satisfying Gaussian bounds and that $S_{t} C_{0} \subseteq L_{\infty ; 1}^{\prime}$ for $t>0$. Therefore $S_{t} C_{0} \subseteq C_{0}$ and since the domain of the generator of $S$ on $L_{\infty}$ contains the norm-dense subspace $C_{0 ; 2}^{\prime}$ of $C_{0}$ the restriction of $S$ to $C_{0}$ is strongly continuous. Now let $\delta_{s}=\left(I-L\left(e^{-s a_{i}}\right)\right) / s$ with $i \in\left\{1, \ldots, d^{\prime}\right\}$. Then

$$
\left\|\left(\delta_{s}-\delta_{u}\right) S_{t} \varphi\right\|_{\infty} \leq\left\|\left(\delta_{s}-\delta_{u}\right) S_{t}^{(n)} \varphi\right\|_{\infty}+2\left(s^{-1}+u^{-1}\right)\left\|S_{t}-S_{t}^{(n)}\right\|_{\infty \rightarrow \infty}\|\varphi\|_{\infty}
$$

where $S^{(n)}$ denotes the approximants used to construct $S$ in the proof of Proposition 11.1. Therefore

$$
\begin{aligned}
\left\|\left(\delta_{s}-\delta_{u}\right) S_{t} \bar{\varphi}\right\|_{\infty} & \leq \sup _{n \geq 1}\left\|\left(\delta_{s}-\delta_{u}\right) S_{t}^{(n)} \varphi\right\|_{\infty} \\
\leq & \sup _{n \geq 1}\left\|\left(s^{-1} \int_{0}^{s} d s_{1} L\left(e^{-s_{1} a_{i}}\right)-u^{-1} \int_{0}^{u} d u_{1} L\left(e^{-u_{1} a_{i}}\right)\right) A_{i} S_{t}^{(n)} \varphi\right\|_{\infty} \\
& \leq \sup _{n \geq 1}^{-1} \int_{0}^{s} d s_{1}\left\|\left(I-L\left(e^{-s_{1} a_{i}}\right)\right) A_{i} S_{t}^{(n)} \varphi\right\|_{\infty} \\
& \quad+\sup _{n \geq 1} u^{-1} \int_{0}^{u} d u_{1}\left\|\left(I-L\left(e^{-u_{1} a_{i}}\right)\right) A_{i} S_{t}^{(n)} \varphi\right\|_{\infty}
\end{aligned}
$$

for each $\varphi \in C_{0}$. But it follows from Proposition 10.4 and the particular construction of the approximants that one has bounds on the last two integrands which are uniform in $n$ and behave like $s_{1}^{\gamma}$ and $u_{1}^{\gamma}$ respectively. Consequently one obtains estimates

$$
\left\|\left(\delta_{s}-\delta_{u}\right) S_{t} \varphi\right\|_{\infty} \leq a_{\gamma, t}\left(s^{-1} \int_{0}^{s} d s_{1} s_{1}^{\gamma}+u^{-1} \int_{0}^{u} d u_{1} u_{1}^{\gamma}\right)\|\varphi\|_{\infty}
$$

for each $\gamma \in\langle 0,1\rangle$. Thus $\delta_{s} S_{t} \varphi$ is uniformly convergent as $s \rightarrow 0$ and one concludes that $S_{t} \varphi \in C_{0 ; 1}^{\prime}$. But it also follows by a similar argument from Proposition 10.4 and the uniform approximation of $S$ by the regularizations $S^{(n)}$ that one has bounds

$$
\left\|(I-L(g)) A_{i} S_{t} \varphi\right\|_{\infty} \leq a t^{-(1-\varepsilon)}\left(|g|^{\prime}\right)^{\gamma}\|\varphi\|_{\infty}
$$

for each $\gamma \in\langle 0,1\rangle$ where $1-\gamma>\varepsilon>0$ and the value of $a$ depends on $\gamma$ and $\varepsilon$.

Next recall that the perturbation series defining $T$ is norm convergent. Moreover, it follows from the second estimate of (11.17) that $T_{t}, t>0$, maps $L_{\infty}$ into the bounded uniformly continuous functions. Now we argue that $T_{t} C_{0}$ consists of functions which vanish at infinity. Since the perturbation series for $T$ converges in norm it suffices to prove that $T_{t}^{(n)} C_{0}$ consists of functions vanishing at infinity for each $n=0,1,2, \ldots$ 
But it follows from the identification $T^{(0)}=S$ and the discussion at the beginning of the proof that $T_{t}^{(0)} C_{0} \subseteq C_{0 ; 1}^{\prime}$ for $t>0$ and we prove by induction that $T_{t}^{(n)} C_{0} \subseteq C_{0 ; 1}^{\prime}$ for all $n=1,2, \ldots$ and $t>0$.

Assume that the induction hypothesis is valid for $n-1$. Then if $\varphi \in C_{0}$ it follows that $H_{1} T_{t}^{(n-1)} \varphi$ vanishes at infinity and since $T^{(0)}=S$ has a Gaussian kernel by (11.4) one has $T_{t-s}^{(0)} H_{1} T_{s}^{(n-1)} \varphi \in C_{0}$ for $s \in\langle 0,1\rangle$. But the norm of the integrand in

$$
T_{t}^{(n)} \varphi=-\int_{0}^{t} d s T_{t-s}^{(0)} H_{1} T_{s}^{(n-1)} \varphi
$$

has an integrable bound. Similarly the integrand in

$$
A_{i} T_{t}^{(n)} \varphi=-\int_{0}^{t} d s A_{i} T_{t-s}^{(0)} H_{1} T_{s}^{(n-1)} \varphi
$$

has an integrable bound. Thus to prove that the two integrals exist in $C_{0}$ it suffices to show that they exist as Bochner integrals in $C_{0}$, i.e., it suffices to show that $s \mapsto T_{t-s}^{(0)} H_{1} T_{s}^{(n-1)} \varphi$ and $s \mapsto A_{i} T_{t-s}^{(0)} H_{1} T_{s}^{(n-1)} \varphi$ are continuous for $s \in\langle 0, t\rangle$. But as $T_{t}^{(0)} C_{0} \subseteq C_{0 ; 1}^{\prime}$ and $T^{(0)}$ is strongly continuous on $C_{0}$ it suffices for the first of these to establish that $s \mapsto H_{1} T_{s}^{(n-1)} \varphi$ is continuous. If $n=0$ this follows because

$$
\left\|H_{1} T_{s}^{(0)} \varphi-H_{1} T_{s+\varepsilon}^{(0)} \varphi\right\|_{\infty} \leq a\|c\|_{\infty} S^{-(1+\delta) / 2} e^{\omega s}\left\|\left(I-T_{\varepsilon}^{(0)}\right) \varphi\right\|_{\infty} .
$$

Then for $n \geq 1$ one has

$$
\begin{aligned}
& H_{1} T_{s}^{(n)} \varphi-H_{1} T_{s+\varepsilon}^{(n)} \varphi=\int_{s}^{s+\varepsilon} d u H_{1} T_{s+\varepsilon-u}^{(0)} H_{1} T_{u}^{(n-1)} \varphi \\
& \quad+\int_{0}^{s} d u H_{1} T_{s-u}^{(0)}\left(T_{\varepsilon}^{(0)}-I\right) H_{1} T_{u}^{(n-1)} \varphi
\end{aligned}
$$

Since $\left\|H_{1} T_{s+\varepsilon-u}^{(0)}\right\|_{\infty \rightarrow \infty} \leq a\|c\|_{\infty}(s+\varepsilon-u)^{-(1+\delta) / 2} e^{\omega(s+\varepsilon-u)}$ the first term on the right is norm convergent to zero as $\varepsilon \rightarrow 0$. But the integrand of the second term tends pointwise to zero and has an integrable bound $a\|c\|_{\infty}^{2}(s-u)^{-(1+\delta) / 2} u^{n(1-\delta) / 2-(1+\delta) / 2} e^{\omega(s+\varepsilon)}$. Therefore the second term is norm convergent to zero by the Lebesgue dominated convergence theorem. Thus $s \mapsto H_{1} T_{s}^{(n)} \varphi$ is continuous and $T_{t}^{(n)} C_{0} \subseteq C_{0}$. The function $s \mapsto A_{i} T_{t-s}^{(0)} H_{1} T_{s}^{(n-1)} \varphi$ is continuous by an analogous argument and hence $T_{t}^{(n)} C_{0} \subseteq C_{0 ; 1}^{\prime}$.

The above estimates establish that the series with terms $T_{t}^{(n)}$ and $A_{i} T_{t}^{(n)}$ are norm convergent for all positive $t$. Thus $T_{t} C_{0} \subseteq C_{0 ; 1}^{\prime}$. Finally $T$ restricted to $C_{0}$ is strongly continuous because $D(H)$ contains the norm dense subset $C_{0 ; 2}^{\prime}$ of $C_{0}$ and the 
estimates (11.18) follow from the similar estimates (11.19) for $S=T^{(0)}$ and the series expansion. Explicitly one has

$$
\begin{aligned}
&\left\|(I-L(g)) A_{i} T_{t}\right\|_{\infty \rightarrow \infty} \leq\left\|(I-L(g)) A_{i} S_{t}\right\|_{\infty \rightarrow \infty} \\
&+\sum_{n \geq 1} \int_{0}^{t} d s\left\|(I-L(g)) A_{i} S_{t-s}\right\|_{\infty \rightarrow \infty}\left\|H_{1} T_{s}^{(n-1)}\right\|_{\infty \rightarrow \infty}
\end{aligned}
$$

and the desired result follows by use of (11.19) and (11.16).

Remark Although Proposition 11.2 establishes that $T$ in restriction to $C_{0}$ is a strongly continuous semigroup it is not necessarily true that $C_{0 ; 2}^{\prime}$ is in the domain of its generator. Since $c_{0}, c_{i}$ and the derivatives $A_{j} c_{i j}$ are not assumed to be continuous the domain of the generator might well have zero intersection with $C_{0 ; 2}^{\prime}$. It is a remarkable consequence of subellipticity that the action of $T$ is much smoother than the coefficients of its generator.

Next we combine the foregoing $L_{\infty}$-estimation techniques with similar $L_{2^{-}}$, and $L_{\hat{2}^{-}}$, estimates to obtain information concerning the action of the semigroup $T$ on the $L_{p^{-}}$, and $L_{\hat{p}^{-}}$, spaces with $p \in[2, \infty]$.

First consider the operator with no first-order terms and let $S^{(n)}$ denote the sequence of approximating semigroups constructed by regularization of the leading coefficients as in the proof of Proposition 11.1. Then $S_{t}^{(n)}$ converges uniformly on $L_{2}$, or $L_{\hat{2}}$, to a semigroup $S$ by the argument given at the beginning on the section. Moreover, $S_{t} L_{2} \subseteq L_{2 ; 1}^{\prime}$ and $S_{t} L_{\hat{2}} \subseteq L_{\hat{2} ; 1}^{\prime}$ for $t>0$ by repetition of the reasoning used to deduce that $S_{t} L_{\infty} \subseteq L_{\infty ; 1}^{\prime}$ in Proposition 11.1. One simply replaces the $L_{\infty}$-estimates by $L_{2^{-}}$, or $L_{\hat{2}^{-}}$, estimates. Similarly one establishes that the generators of $S$ on $L_{2}$ and $L_{\hat{2}}$ extend $H$.

Secondly, one estimates the norm of $S_{t}$ or its derivatives $\partial_{t}^{k} S_{t}$ on $L_{2}$ and $L_{\hat{2}}$ by the methods of Section 10 after an initial approximation with the semigroups $S^{(n)}$. Finally these estimates lead to norm bounds on $A_{i} S_{t}$ either on $L_{\hat{2}}$ or as an operator from $L_{\hat{2}}$ to $L_{\infty}$. Specifically one has bounds

$$
\begin{aligned}
& \left\|S_{t}\right\|_{\hat{2} \rightarrow \hat{2}} \leq a e^{\omega t}, \quad\left\|A_{i} S_{t}\right\|_{\hat{2} \rightarrow \hat{2}} \leq a t^{-1 / 2} e^{\omega t}, \\
& \left\|S_{t}\right\|_{\hat{2} \rightarrow \infty} \leq a t^{-D^{\prime} / 4} e^{\omega t} \quad\left\|A_{i} S_{t}\right\|_{\hat{2} \rightarrow \infty} \leq a_{\delta} t^{-D^{\prime} / 4-(1+\delta) / 2} e^{\omega t} \text {, }
\end{aligned}
$$

for all $t>0$ where the values of $a>0$ and $\omega \geq 0$ depend on the coefficients of $H$ only 
through $\lambda_{c}^{-}$and the norm $\|c\|_{1}^{\prime}$ which in the current context can be defined as

$$
\|c\|_{1}^{\prime}=\sum_{i, j=1}^{d^{\prime}}\left\|c_{i j}\right\|_{\infty ; 1}^{\prime}+\sum_{i=1}^{d^{\prime}}\left\|c_{i}\right\|_{\infty}+\left\|c_{0}\right\|_{\infty} .
$$

Similar estimates are also valid for the $L_{2}$-spaces.

Thirdly, the estimates on $S_{t}$ and $A_{i} S_{t}$ acting on $L_{2}$ or $L_{\hat{2}}$ allow one to construct the semigroup $T$, generated by an extension of the full operator with first-order terms, by the perturbation expansion used on $L_{\infty}$ in Propositions 11.1 and 11.2. But then the crossnorm estimates on $S$ can be used to obtain similar estimates on $T$ and hence to conclude that $T$ is determined by a semigroup kernel with some basic integrability and regularity properties.

Proposition_11.3 Let $H$ be a subelliptic operator in the form

$$
H=-\sum_{i, j=1}^{d^{\prime}} A_{i} c_{i j} A_{i}+\sum_{i=1}^{d^{\prime}} c_{i} A_{i}+c_{0}
$$

with coefficients $c_{i j} \in L_{\infty ; 1}^{\prime}$ and $c_{i}, c_{0} \in L_{\infty}$ and let $T$ denote the corresponding semigroup on $L_{\infty}$.

Then $T$ interpolates consistently between the $L_{p^{-}}$, and $L_{\hat{p}^{-}}$, spaces for $p \in[2, \infty]$. The generator of $T$ on each of the spaces is an extension of $H$ and its action is determined by a positive kernel $K_{t} \in \mathcal{L}_{\hat{1}} \cap \mathcal{L}_{\hat{2}}$.

Proof The estimates (11.14) on $S_{t}$ and $A_{i} S_{t}$ acting on $L_{\infty}$ together with the above estimates for the operators on $L_{\hat{2}}$ and from $L_{\hat{2}}$ to $L_{\infty}$ suffice to deduce that the semigroup $T$ constructed by perturbation theory from $S$ and $H_{1}$ is bounded from $L_{\hat{2}}$ to $L_{\infty}$. This is a direct consequence of the proposition in the appendix and in fact one obtains bounds

$$
\left\|T_{t}\right\|_{\hat{2} \rightarrow \infty} \leq a t^{-D^{\prime} / 4} e^{\omega t} \quad, \quad\left\|A_{i} T_{t}\right\|_{\hat{2} \rightarrow \infty} \leq a_{\delta} t^{-D^{\prime} / 4-(1+\delta) / 2} e^{\omega t}
$$

for all $\delta, t>0$.

Now as

$$
\left|\left(T_{t} \varphi\right)(g)\right| \leq\left\|T_{t}\right\|_{\hat{2} \rightarrow \infty}\|\varphi\|_{\hat{2}}
$$

for all $\varphi \in L_{\hat{2}}$ it follows from the Riesz representation theorem that there exists a $K_{t} \in \mathcal{L}_{\hat{2}}$ such that

$$
\left(T_{t} \varphi\right)(g)=\int_{G} d \hat{h} K_{t}(g ; h) \varphi(h)
$$


for all $\varphi \in L_{\hat{2}}$. But $T_{t}$ is bounded on $L_{\infty}$ and hence one must have $K_{t} \in \mathcal{L}_{\hat{1}}$ and

$$
\|\| K_{t}\left\|_{\hat{1}}=\right\| T_{t} \|_{\infty \rightarrow \infty} .
$$

It follows automatically from (11.21) with $K_{t} \in \mathcal{L}_{\hat{1}} \cap \mathcal{L}_{\hat{2}}$ that $T$ interpolates between the $L_{\hat{p}}$-spaces with $p \in[2, \infty]$. Since $T$ is bounded from $L_{2}$ to $L_{\infty}$ a similar conclusion is valid relative to the $L_{p}$-spaces and in fact one has the additional integral bound

$$
\left\|T_{t}\right\|_{2 \rightarrow \infty}=\underset{g \in G}{\operatorname{esssup}}\left(\int_{G} d \hat{h}\left|K_{t}(g ; h)\right|^{2} \Delta^{-1}(h)\right)^{1 / 2} \leq a t^{-D^{\prime} / 4} e^{\omega t} .
$$

Moreover, as the generator of $T$ on $L_{\hat{2}}$ and $L_{\infty}$ extends $H$ it is easily deduced that this is also true for $T$ acting on $L_{\hat{p}}$ or $L_{p}, p \in\langle 2, \infty\rangle$.

Finally the kernel $K$ is positive as a consequence of the arguments of [ABR].

The cross-norm estimates (11.20) immediately give bounds on the $\mathcal{L}_{\hat{2}}$-norm of the kernel and its derivatives. Similarly the analogous bounds

$$
\left\|T_{t}\right\|_{\infty \rightarrow \infty} \leq a e^{\omega t} \quad, \quad\left\|A_{i} T_{t}\right\|_{\infty \rightarrow \infty} \leq a_{\delta} t^{-(1+\delta) / 2} e^{\omega t}
$$

give bounds on the $\mathcal{L}_{\hat{1}}$-norms.

Corollary 11.4 Let $H$ be a subelliptic operator in the form

$$
H=-\sum_{i, j=1}^{d^{\prime}} A_{i} c_{i j} A_{i}+\sum_{i=1}^{d^{\prime}} c_{i} A_{i}+c_{0}
$$

with coefficients $c_{i j} \in L_{\infty ; 1}^{\prime}$ and $c_{i}, c_{0} \in L_{\infty}$ and let $K \in \mathcal{L}_{\hat{1}} \cap \mathcal{L}_{\hat{2}}$ denote the corresponding semigroup kernel.

There exist $a, a_{\delta}>0$ and $\omega \geq 0$ such that

$$
\begin{array}{ll}
\left\|K_{t}\right\|_{\hat{1}} \leq a e^{\omega t}, & \left\|A_{i} K_{t}\right\|_{\hat{1}} \leq a_{\delta} t^{-(1+\delta) / 2} e^{\omega t}, \\
\left\|K_{t}\right\|_{\hat{2}} \leq a t^{-D^{\prime} / 4} e^{\omega t}, \quad\left\|A_{i} K_{t}\right\|_{\hat{2}} \leq a t^{-D^{\prime} / 4-(1+\delta) / 2} e^{\omega t},
\end{array}
$$

for all $t>0$ and $i \in\left\{1, \ldots, d^{\prime}\right\}$ where the values of $a, a_{\delta}$ and $\omega$ depend on the coefficients of $H$ only through $\lambda_{c}$ and $\|c\|_{1}^{\prime}$.

Our next aim is to strengthen the statement of the foregoing propositions by using another approximation with first-order coefficients which are differentiable in the subelliptic directions. This condition is extremely useful because the adjoint operator can then be expressed in the same form and hence by duality one may obtain 
$L_{1}$-estimates from the $L_{\infty}$-estimates. First we need to derive extra information on the semigroups for which the first-order coefficients are smooth. Subsequently this will be used in the approximation argument.

There are two distinct ways of proceeding. Either one may continue to analyze the semigroup $T$ defined by the perturbation expansion from the semigroup $S$ with no first-order terms, or one may exploit the extra smoothness of the first-order terms to extend the approximation arguments used to construct $S$. We choose the latter tactic to prove the next result.

Proposition 11.5 Let $H$ be a subelliptic operator in the form

$$
H=-\sum_{i, j=1}^{d^{\prime}} A_{i} c_{i j} A_{i}+\sum_{i=1}^{d^{\prime}}\left(c_{i} A_{i}+A_{i} c_{i}^{\prime}\right) / 2+c_{0}
$$

with coefficients $c_{i j}, c_{i}, c_{i}^{\prime} \in L_{\infty ; 1}^{\prime}$ and $c_{0} \in L_{\infty}$.

Then $H$, on $L_{\infty}$, has a closed extension $\widetilde{H}$ that generates a semigroup $T$ which interpolates consistently between the $L_{p^{-}}$, and $L_{\hat{p}^{-}}$, spaces for all $p \in[1, \infty]$. The semigroup is holomorphic and has a positive kernel $K \in \mathcal{L}_{\infty}$. The function $t>0 \mapsto$ $K_{t} \in \mathcal{L}_{\infty}$ is analytic and $K_{t}$ together with its derivatives with respect to $t$ lies in $\mathcal{L}_{\infty ; 1}^{\prime}$. Moreover, there exist $a, b>0$ and $\omega \geq 0$ such that

$$
K_{t}(g ; h) \leq a t^{-D^{\prime} / 2} e^{\omega t} e^{-b\left(\left|g h^{-1}\right|^{\prime}\right)^{2} t^{-1}}
$$

for all $g, h \in G$ and $t>0$. The values of $a, b$ and $\omega$ depend on the coefficients of $H$ only through $\lambda_{c}$ and the norm $\||c|\|$ of Section 10.

Proof We again construct approximations $H_{n}$ to $H$ by replacing the $c_{i j}$ and the $c_{i}, c_{i}^{\prime}$ with right regularizations $c_{i j}^{(n)}$ and $c_{i}^{(n)}, c_{i}^{\prime(n)}$ defined as in the proof of Proposition 11.1. Then we argue as above that the corresponding sequence of semigroups $S^{(n)}$ converges in norm on $L_{\hat{2}}$ or $L_{2}$ and the convergence is uniform for $t \in[0,1]$. The proof is identical to the proof for the symmetric case because the adjoint of $H_{n}$ with respect to right Haar measure is of the same form as $H_{n}$ and its coefficients satisfy the same assumptions. The only significant difference is that the zero-order term $c_{0}^{(n)}$ of the adjoint varies with $n$. Nevertheless, $c_{0}^{(n)}$ is a linear combination of the $c_{i j}^{(n)}$, the $c_{i}^{(n)}, c_{i}^{\prime(n)}$ and the original $c_{0}$. Since the $c_{i j}, c_{i}, c_{i}^{\prime} \in L_{\infty ; 1}^{\prime}$ it follows, however, that $c_{0}^{(n)}$ is $L_{\infty}$-convergent as $n \rightarrow \infty$. The estimates are again expressed in terms of $\lambda_{c}$ and the norm

$$
C_{1}=\sum_{i, j=1}^{d^{\prime}}\left\|c_{i j}\right\|_{\infty ; 1}^{\prime}+\sum_{i=1}^{d^{\prime}}\left(\left\|c_{i}\right\|_{\infty ; 1}^{\prime}+\left\|c_{i}^{\prime}\right\|_{\infty ; 1}^{\prime}\right)+\left\|c_{0}\right\|_{\infty}
$$


It also follows by repetition of the previous arguments that the kernels $K^{(n)}$ associated with the $S^{(n)}$ converge uniformly on $\mathcal{L}_{\hat{1}}$. But

$$
K_{t}^{(n)}(h ; g)=\tilde{K}_{t}^{(n)}(g ; h)
$$

where $\tilde{K}^{(n)}$ is the kernel of the semigroup $\tilde{S}^{(n)}$ which is adjoint to $S^{(n)}$ on the $L_{\hat{p}^{-}}$ spaces. Therefore the previous reasoning applied to $\tilde{S}^{(n)}$ gives

$$
\lim _{n, m \rightarrow \infty} \operatorname{esssup}_{h \in G} \int_{G} d \hat{g}\left|K_{t}^{(n)}(g ; h)-K_{t}^{(m)}(g ; h)\right|=0
$$

and this implies that

$$
\lim _{n, m \rightarrow \infty}\left\|S_{t}^{(n)}-S_{t}^{(m)}\right\|_{\hat{1} \rightarrow \hat{1}}=0 .
$$

Since one already has convergence on $L_{\infty}$ it follows by interpolation that

$$
\lim _{n, m \rightarrow \infty}\left\|S_{t}^{(n)}-S_{t}^{(m)}\right\|_{\hat{p} \rightarrow \hat{p}}=0
$$

for all $p \in[1, \infty]$. But the $S^{(n)}$ are interpolating semigroups on the $L_{\hat{p}^{-} \text {-spaces and }}$ hence their uniform limits $T$ also form a continuous interpolating semigroup.

Next remark that it also follows from (11.13) and (11.4) that

$$
\lim _{n, m \rightarrow \infty}\left\|K_{t}^{(n)}-K_{t}^{(m)} \mid\right\|_{\infty}=0 .
$$

The proof is by the a repetition of the reasoning used at the beginning of the section to obtain the $\||\cdot|\|_{\hat{2}}$-convergence of the kernels from the $\|\cdot\|_{\hat{2}}$-convergence of the semigroups, i.e., from (11.2). Then since the approximating semigroups $S^{(n)}$ have positive kernels $K^{(n)}$ which satisfy Gaussian bounds uniformly in $n$, by Proposition 10.1, it follows that the $\mathcal{L}_{1}$-limit $K$ of the $K^{(n)}$ is the kernel for $S$ and that it is positive and has a Gaussian bound with the various parameters in the bound dependent on the coefficients only through $\lambda_{c}$ and the norm $\||c|\|$.

Now we consider the generator of $S$. It follows from Proposition 11.3 that the generator of $T$ on $L_{p}$ or $L_{\hat{p}}$ with $p \in[2, \infty]$ is an extension of $H$ but the result for all $p \in[1, \infty\rangle$ can also be established by the following approximation argument.

If $\omega=\left\|c_{0}\right\|_{\infty}+\sum_{i=1}^{d^{\prime}}\left\|\left(A_{i} c_{i}^{\prime}\right)\right\|_{\infty} / 2$ then $H_{n}-\omega I$ is dissipative on $L_{\infty}$. Moreover, one can choose $\tilde{\omega}$ large enough that $\tilde{H}_{n}-\tilde{\omega} I$ is also dissipative on $L_{\infty}$ where $\tilde{H}_{n}$ is the adjoint of $H_{n}$ with respect to right Haar measure. Thus with $\omega^{\prime}=\omega \vee \tilde{\omega}$ one has

$$
\left\|S_{t}^{(n)}\right\|_{\hat{p} \rightarrow \hat{p}} \leq e^{\omega^{\prime} t}
$$


by interpolation. Hence the generator $\widetilde{H}$ of $T$ on $L_{p}$ for $p \in[1, \infty\rangle$ is the graph limit of the $H_{n}$ as a corollary of the approximation theory of strongly continuous contraction semigroups (see, for example, [BrR2] Theorem 3.1.28). Explicitly, $\psi \in D(\widetilde{H})$ if and only if there is a $\chi$ and a sequence $\psi_{n} \in D\left(H_{n}\right)$ such that $\left\|\psi_{n}-\psi\right\|_{\hat{p}} \rightarrow 0$ and $\left\|H_{n} \psi_{n}-\chi\right\|_{\hat{p}} \rightarrow 0$. Then $\chi=\widetilde{H} \psi$. Now we establish that $\left\|\left(H_{n}-H\right) \psi\right\|_{\hat{p}} \rightarrow 0$ for all $\psi \in L_{\hat{p} ; 2}^{\prime}$ and consequently conclude that $\widetilde{H}$ extends $H$.

First observe that $\left\|\left(H_{n}-H\right) \psi\right\|_{\hat{p}} \leq C\|\psi\|_{\hat{p} ; 2}^{\prime}$ for a suitable $C>0$. Therefore it suffices to prove convergence on the dense subset $C_{c}^{\infty}$ of $L_{\hat{p} ; 2}^{\prime}$. But if $\psi \in C_{c}^{\infty}$ then one has

$$
\begin{gathered}
\left\|\left(H_{n}-H\right) \psi\right\|_{\hat{p}} \leq\left\|c^{(n)}-c\right\|_{\infty}\|\psi\|_{\hat{p} ; 2}^{\prime}+\sum_{i, j=1}^{d^{\prime}}\left\|\left(\left(A_{i} c_{i j}^{(n)}\right)-\left(A_{i} c_{i j}\right)\right) A_{j} \psi\right\|_{\hat{p}} \\
+\sum_{i=1}^{d^{\prime}}\left\|\left(\left(A_{i} c_{i}^{(n)}\right)-\left(A_{i} c_{i}^{\prime}\right)\right) \psi\right\|_{\hat{p}} / 2
\end{gathered}
$$

where

$$
\left\|c^{(n)}-c\right\|_{\infty}=\sum_{i, j=1}^{d^{\prime}}\left\|c_{i j}^{(n)}-c_{i j}\right\|_{\infty}+\sum_{i=1}^{d^{\prime}}\left(\left\|c_{i}^{(n)}-c_{i}\right\|_{\infty}+\left\|c_{i}^{\prime(n)}-c_{i}^{\prime}\right\|_{\infty}\right) .
$$

Thus the first term on the right-hand side of (11.26) tends to zero as $n \rightarrow \infty$. Next we argue that the same is true for the other terms provided that $p \in[1, \infty\rangle$. All the other terms are of the form $\left\|\left(A_{i} c^{(n)}-A_{i} c\right) \psi\right\|_{\hat{p}}$ where $c \in L_{\infty ; 1}^{\prime}$ and $\psi \in C_{c}^{\infty}$. But $A_{i} c^{(n)}=\left(A_{i} c\right)^{(n)}$ since $A_{i}$ is a left derivative and $c$ is regularized with respect to right translations. Thus it suffices to establish that

$$
\lim _{n \rightarrow \infty}\left\|\left(c^{(n)}-c\right) \psi\right\|_{\hat{p}}=0
$$

whenever $c \in L_{\infty}$ and $\psi \in C_{c}^{\infty}$. But

$$
\begin{aligned}
\left(c^{(n)}-c\right) \psi(g) & =\int_{G} d h \varphi_{n}(h)(c(g h)-c(g)) \psi(g) \\
& =\int_{G} d h \varphi_{n}(h)(c(g h)(\psi(g)-\psi(g h))-(c(g) \psi(g)-c(g h) \psi(g h)))
\end{aligned}
$$

and hence

$$
\left\|\left(c^{(n)}-c\right) \psi\right\|_{\hat{p}} \leq \int_{G} d h \varphi_{n}(h)\left(\|c\|_{\infty}\|(I-R(h)) \psi\|_{\hat{p}}+\|(I-R(h)) c \psi\|_{\hat{p}}\right) .
$$

Since right translations are strongly continuous on $L_{\hat{p}}$ for $p \in[1, \infty\rangle$ it follows that the right hand side tends to zero as $n \rightarrow \infty$. Thus $\widetilde{H}$ extends $H$ on the spaces $L_{\hat{p}}$ with $p \in[1, \infty)$. 
The holomorphy of $S$ on $L_{\infty}$ was established in Proposition 11.1 and the same proof applies to the adjoint group acting on $L_{\infty}$. Thus by duality $S$ is holomorphic on $L_{\hat{1}}$ and then by interpolation on all the $L_{\hat{p}}$-spaces. Moreover, this argument establishes the existence of a $p$-independent sector of holomorphy.

The smoothness properties of $K$ are deduced by the same arguments used to discuss the kernels associated with operators with smooth coefficients in Section 10.

Finally, the existence of $S$ on the $L_{p}$-spaces is established by similar but simpler reasoning.

Note that although the proof of convergence of the approximating semigroups in the above proof relied upon the differentiability of the first-order coefficients the parameters in the Gaussian bounds do not depend on these derivatives. This is a consequence of the estimates of Section 10 and it is crucial for the final stage in our approximation procedure.

One can derive Gaussian bounds on the derivatives $\partial_{t}^{n} K_{t}$ similar to the bounds (10.19) for smooth coefficients. Again one uses approximants $S^{(n)}$ for the semigroup but now one must also consider complex values of $t$. This, however, presents no problem. In addition one can derive uniform bounds on the left derivatives $A_{i} K_{t}$ of the kernel with respect to the first variable. These are the analogue of (10.21) and supplement the bounds of Corollary 11.4 and Proposition 11.5.

Proposition 11.6 Let $H$ be a subelliptic operator in the form

$$
H=-\sum_{i, j=1}^{d^{\prime}} A_{i} c_{i j} A_{i}+\sum_{i=1}^{d^{\prime}}\left(c_{i} A_{i}+A_{i} c_{i}^{\prime}\right) / 2+c_{0}
$$

with coefficients $c_{i j}, c_{i}, c_{i}^{\prime} \in L_{\infty ; 1}^{\prime}$ and $c_{0} \in L_{\infty}$ and let $K$ denote the corresponding semigroup kernel.

There exists $a_{\delta}>0$ and $\omega \geq 0$ such that

$$
\left\|\mid A_{i} K_{t}\right\|_{\infty} \leq a_{\delta} t^{-D^{\prime} / 2} t^{-(1+\delta) / 2} e^{\omega t}
$$

for $i \in\left\{1, \ldots, d^{\prime}\right\}$ and for all $\delta, t>0$. The values of $a_{\delta}$ and $\omega$ depend on the coefficients of $H$ through $\lambda_{c}$ and the norm

$$
\|c \mid\|_{1}=\sum_{i, j=1}^{d^{\prime}}\left\|c_{i j}\right\|_{\infty ; 1}+\sum_{i=1}^{d^{\prime}}\left(\left\|c_{i}\right\|_{\infty}+\left\|c_{i}^{\prime}\right\|_{\infty ; 1}\right)+\left\|c_{0}\right\|_{\infty}
$$


Proof Let $S^{(n)}$ be the approximants to the semigroup $T$ and again set $\delta_{s}=(I-$ $\left.L\left(e^{-s a_{i}}\right)\right) / s$. Then

$$
\begin{aligned}
\left\|\delta_{s} T_{t} \varphi\right\|_{\infty} & \leq\left\|\delta_{s} S_{t}^{(n)} \varphi\right\|_{\infty}+2 s^{-1}\left\|\left(T_{t}-S_{t}^{(n)}\right) \varphi\right\|_{\infty} \\
& \leq\left\|A_{i} S_{t}^{(n)} \varphi\right\|_{\infty}+2 s^{-1}\left\|\left(T_{t}-S_{t}^{(n)}\right) \varphi\right\|_{\infty} .
\end{aligned}
$$

Hence

$$
\left\|\delta_{s} T_{t} \varphi\right\|_{\infty} \leq \sup _{n \geq 1}\left\|A_{i} S_{t}^{(n)} \varphi\right\|_{\infty}
$$

Therefore one has

$$
\left\|A_{i} K_{t}\right\|_{\infty}=\left\|A_{i} T_{t}\right\|_{\hat{1} \rightarrow \infty} \leq \sup _{n \geq 1}\left\|A_{i} S_{t}^{(n)}\right\|_{\hat{i} \rightarrow \infty}
$$

But the last term on the right has the appropriate bound by Proposition 10.3.

Note that although we have assumed in this proposition that the coefficients of the first-order terms are differentiable the estimates do not depend on the derivatives of the $c_{i}$ but only the $c_{i}^{\prime}$.

Our final aim is to derive the consequences of Proposition 11.5 from the hypotheses of Proposition 11.1 and hence improve the partial information on the semigroup kernel given in Proposition 11.3. Again we use approximation techniques.

Let $T$ denote the semigroup with generator $\widetilde{H}$ constructed in Propositions 11.1 and 11.3 and $K$ the corresponding semigroup kernel. The semigroup is given in terms of the semigroup $S$ with no first-order terms by the 'time-dependent' perturbation expansion which is convergent on $L_{\infty}$ and $L_{\hat{2}}$. Moreover, the perturbation estimates discussed in the appendix and used in the proof of Proposition 11.3 show that $T$ and the $A_{i} T$ are bounded operators not only on $L_{\hat{2}}$ but as operators from $L_{\hat{2}}$ to $L_{\infty}$ satisfying the estimates (11.20). But the second of these estimates is equivalent to the statement that

$$
\|\| A_{i} K_{t} \|_{\hat{2}} \leq a t^{-D^{\prime} / 4} t^{-(1+\delta) / 2} e^{\omega t}
$$

for all $t>0$ and the values of $a$ and $\omega$ depend on the coefficients only through $\lambda_{c}$ and $\|c\|_{1}^{\prime}$.

Next let $c_{i}^{(n)}$ denote the regularizations of the first-order coefficients as used in the proof of Proposition 11.4. and set

$$
H_{1, n}=\sum_{i=1}^{d^{\prime}}\left(c_{i}^{(n)}-c_{i}\right) A_{i} \text {. }
$$


Then

$$
\left\|H_{1, n} T_{t}\right\|_{\hat{2} \rightarrow \hat{2}} \leq 2\|c\|_{\infty} a t^{-1 / 2} e^{\omega t}
$$

and one can construct semigroups $T^{(n)}$ with generators $\widetilde{H}+H_{1, n}$, i.e., generators with regularized first-order terms $\sum_{i=1}^{d^{\prime}} c_{i}^{(n)} A_{i}$, by perturbation from $T$. If $K^{(n)}$ denotes the corresponding kernels then it follows from Proposition 11.5 and the construction of the regularizations that one has estimates

$$
\left\|\mid A_{i} K_{t}^{(n)}\right\|_{\hat{2}} \leq a_{\delta}^{\prime}\left(\lambda_{c} t\right)^{-D^{\prime} 4} t^{-(1+\delta) / 2} e^{\omega t}
$$

uniform in $n$ where $a_{\delta}^{\prime}$ and $\omega$ again depend on the coefficients through $\lambda_{c}$ and the norm $\|c\|_{1}$ given by (11.28). Since, however, we are now assuming that $c_{i}^{\prime}=0$ this latter norm does not-depend on the derivatives of the first-order terms and one has $\||c|\|_{1}=\|\bar{c}\|_{1}^{\prime}$. In conclusion, the estimates on $\left\|\left|A_{i} K_{t} \|\right|_{\hat{2}}\right.$ and $\|\left|A_{i} K_{t}^{(n)}\right| \|_{\hat{2}}$ only depend on $\lambda_{c}$ and $\|c\|_{1}^{\prime}$ and the latter estimates are uniform in $n$.

The next important point is that the semigroups $T^{(n)}$ converge strongly to $T$ on $L_{\hat{2}}$. This is established by a Duhamel estimate

$$
\left\|\left(T_{t}^{(n)}-T_{t}\right) \varphi\right\|_{\hat{2}} \leq \sum_{i=1}^{d^{\prime}} \int_{0}^{t} d s\left\|T_{t-s}^{(n)}\right\|_{\hat{2} \rightarrow \hat{2}}\left\|\left(c_{i}^{(n)}-c_{i}\right) A_{i} T_{s} \varphi\right\|_{\hat{2}} .
$$

Now $\left\|T_{t}^{(n)}\right\|_{\hat{2} \rightarrow \hat{2}}$ is bounded uniformly in $n$ on each compact subinterval of $\mathbf{R}_{+}$and the bound only depends on the coefficients through $\lambda_{c}$ and $\||| c||$ by (10.4). Moreover, $c_{i}^{(n)}$ converges strongly to $c_{i}$ on $L_{\hat{2}}$ by the argument given in the proof of Proposition 11.5. Finally, one has bounds

$$
\left\|\left(c_{i}^{(n)}-c_{i}\right) A_{i} T_{s} \varphi\right\|_{\hat{2}} \leq a\left\|c_{i}\right\|_{\infty} s^{-1 / 2} e^{\omega s}\|\varphi\|_{\hat{2}}
$$

uniform in $n$ with the values of $a$ and $\omega$ depending on the coefficients through $\lambda_{c}$ and $\|c\|_{1}^{\prime}$. Therefore $\left\|\left(T_{t}^{(n)}-T_{t}\right) \varphi\right\|_{\hat{2}} \rightarrow 0$ as $n \rightarrow \infty$ uniformly for $t$ in intervals $\left[0, t_{0}\right]$ as a consequence of the Lebesgue dominated convergence theorem.

Next remark that the $L_{\hat{2}}$-convergence of the semigroups and the uniform bounds on $\left\|\left|A_{i} K_{t}^{(n)}\right|\right\|_{\hat{2}}$ and $\left\|\mid A_{i} K_{t}\right\|_{\hat{2}}$ suffice to prove that

$$
\lim _{n \rightarrow \infty} \mid\left\|K_{t}^{(n)}-K_{t}\right\|_{\hat{2}}=0 \text {. }
$$

This is a consequence of the argument which proved that (11.6) followed from (11.2) and (11.5). It is the vital observation for the proof of the next proposition. 
Proposition 11.7 Let $H$ be a subelliptic operator in the form

$$
H=-\sum_{i, j=1}^{d^{\prime}} A_{i} c_{i j} A_{i}+\sum_{i=1}^{d^{\prime}} c_{i} A_{i}+c_{0}
$$

with coefficients $c_{i j} \in L_{\infty ; 1}^{\prime}$ and $c_{i}, c_{0} \in L_{\infty}$ and let $K_{t} \in \mathcal{L}_{\hat{1}} \cap \mathcal{L}_{\hat{2}}$ denote the positive kernel of the semigroup $T$ constructed in Proposition 11.3.

Then $K_{t} \in \mathcal{L}_{\infty}$, the function $t>0 \mapsto K_{t} \in \mathcal{L}_{\infty}$ is analytic and $K_{t}$ together with its derivatives with respect to $t$ lies in $\mathcal{L}_{\infty ; 1}^{\prime}$ and there exist $a, b>0$ and $\omega \geq 0$ such that

$$
0 \leq K_{t}(g ; h) \leq a t^{-D^{\prime} / 2} e^{\omega t} e^{-b\left(\left|g h^{-1}\right|^{\prime}\right)^{2} t^{-1}}
$$

for all $g, h \in G$ and $t>0$ where the values of $a, b$ and $\omega$ depend on the coefficients of $H$ only through $\lambda_{c}$ and the norm $\||c|\|$ of Section 10.

Proof The kernels $K^{(n)}$ satisfy Gaussian bounds by Proposition 11.5 and these bounds are uniform in $n$. Let $G_{t}$ denote the Gaussian bound. Then $L_{t}^{(n)}=G_{t}-K_{t}^{(n)} \in$ $\mathcal{L}_{\hat{2}}$ is a positive sequence which is $\mathcal{L}_{\hat{2}}$-convergent to $L_{t}=G_{t}-K_{t} \in \mathcal{L}_{\hat{2}}$ by the foregoing reasoning. Therefore $L_{t}$ is positive and one has $0 \leq K_{t} \leq G_{t}$. It was, however, already established in Proposition 11.5 that the parameters in $G_{t}$ depend on the coefficients of $H$ only through $\lambda_{c}$ and the norm $\||c|\|$.

Again the smoothness properties of $K$ are deduced by the same arguments used to discuss the kernels associated with operators with smooth coefficients in Section 10 .

Finally we prove the interpolating and holomorphy properties of $T$ by exploiting information on the kernels.

Proposition 11.8 Let $H$ be a subelliptic operator in the form

$$
H=-\sum_{i, j=1}^{d^{\prime}} A_{i} c_{i j} A_{i}+\sum_{i=1}^{d^{\prime}} c_{i} A_{i}+c_{0}
$$

with coefficients $c_{i j} \in L_{\infty ; 1}^{\prime}$ and $c_{i}, c_{0} \in L_{\infty}$ and let $T$ denote the corresponding semigroup on $L_{\infty}$.

Then the semigroup $T$ interpolates between the $L_{p^{-}}$, and the $L_{\hat{p}^{-}}$, spaces for $p \in$ $[1, \infty]$ and is holomorphic on each of these spaces. 
Proof Since the kernel of $T$ satisfies Gaussian bounds, by the previous proposition, one can extend its action to all the $L_{p}$-spaces. The extended action automatically has the correct interpolating properties.

Next remark that the semigroup $T$ was approximated in two steps described in the proofs of Propositions 11.3 and 11.6 by semigroups $T^{(n)}$ with $C^{\infty}$-coefficients, Moreover, the corresponding kernels satisfy

$$
\lim _{n \rightarrow \infty}\|\| K_{t}^{(n)}-K_{t} \|_{\hat{2}}=0
$$

Now $T$ can be extended to $\mathcal{L}_{\hat{2}}=L_{\infty} \otimes L_{\hat{2}}$ by identifying it with $T \otimes I$ and since $T$ is holomorphic on $L_{\infty}$, by Proposition 11.3, it follows that its extension on $\mathcal{L}_{\hat{2}}$ is also holomorphic. But as $T_{s} K_{t}=K_{t+s}$ it follows immediately that $K_{t}$ is $\mathcal{L}_{\hat{2}}$-differentiable. Then, however,

$$
\begin{aligned}
\partial_{t} K_{t} & =\lim _{s \rightarrow 0} \lim _{n \rightarrow \infty} s^{-1}\left(K_{t+s}^{(n)}-K_{s}^{(n)}\right) \\
& =\lim _{s \rightarrow 0} \lim _{n \rightarrow \infty} s^{-1} \int_{t}^{t+s} d u \partial_{u} K_{u}^{(n)}
\end{aligned}
$$

where the limits are in the $\mathcal{L}_{\hat{2}}$-sense. Since the derivatives $\partial_{u} K_{u}^{(n)}$ have bounds $a u^{-1} G_{u}$ where $G_{u}$ is a Gaussian in the difference variable it follows that

$$
\left|\partial_{u} K_{u}^{(n)}(g ; h)\right| \leq a u^{-1} G_{u}\left(g h^{-1}\right) .
$$

Therefore

$$
\begin{aligned}
\left\|H T_{t}\right\|_{\hat{\mathfrak{I}}} & \leq \sup _{g \in G} \int_{G} d \hat{g}\left|\partial_{t} K_{t}^{(n)}(g ; h)\right| \cdot\|\varphi\|_{\hat{1}} \\
& \leq c t^{-1}\|\varphi\|_{\hat{1}}
\end{aligned}
$$

for $t \in\langle 0,1]$ where the last estimate uses the Gaussian bounds. Thus $T$ is holomorphic on $L_{\hat{1}}$ and by interpolation on each $L_{\hat{p}}$. Similarly $T$ is holomorphic on $L_{1}$ and then on each $L_{p}$.

It is a consequence of the foregoing proof that one has bounds

$$
\left|\partial_{t} K_{t}(g ; h)\right| \leq a t^{-1} G_{t}\left(g h^{-1}\right)
$$

on the derivatives of the kernel of the semigroup T. But it is not difficult to extend the proof to obtain bounds

$$
\left|\partial_{t}^{n} K_{t}(g ; h)\right| \leq a^{n} t^{-n} G_{t}\left(g h^{-1}\right) .
$$


on the higher derivatives. These clearly demonstrate that $K$ is an analytic function of $t$.

Remarks 1. The disadvantage with the foregoing propositions and other generator results derived from them is the failure to identify the generator $\widetilde{H}$ of the semigroup with the closure of $H$. Thus in the language of partial differential equations the propositions provide existence of a solution of an evolution problem but not uniqueness. One can, however, establish somewhat more about the generator extension on $L_{2}$ or $L_{\hat{2}}$ under the hypotheses of Proposition 11.5. Since $\widetilde{H}$ is the graph limit of the $H_{n}$ it follows from Proposition 3.1 by another limiting argument on $L_{2}$ that one has $D(\widetilde{H}) \subseteq L_{2 ; 1}^{\prime}$. Using this information one can then identify $\widetilde{H}+\lambda I$ with the operator determined by the $m$-sectorial form defined by

$\varphi \mapsto h(\varphi)=\sum_{i, j=1}^{d^{\prime}}\left(A_{i} \varphi, c_{i j} A_{j} \varphi\right)+\sum_{i=1}^{d^{\prime}}\left(\left(c_{i} \varphi, A_{i} \varphi\right)+\left(A_{i} \varphi, c_{i} \varphi\right)\right) / 2+\left(\varphi,\left(c_{0}+\lambda I\right) \varphi\right)$

where $\lambda>0$ is chosen sufficiently large to ensure that $h$ takes values in the right-half plane. The theory of sectorial forms gives another possible approach to the generator problem which should provide results with very little smoothness required for the coefficients. The difficulty is to extend the $L_{2}$ properties to the $L_{p}$-spaces and for this one would need a generalization of the theory of Dirichlet forms to $m$-sectorial forms. Results of this nature have been given in the strongly elliptic case by Ouhabaz [Ouh].

2. The semigroup $S$ defined by $H_{0}$ with no first order terms acting on $L_{2}$ is self-adjoint and holomorphic in the open right half plane $\Pi$. Therefore $T$ on $L_{2}$ is also holomorphic in $\Pi$ by the perturbation theory of holomorphic semigroups. A similar conclusion is expected for $T$ on the $L_{p^{-}}$, and $L_{\hat{p}^{-}}$, spaces but this does not follow from the above arguments. If the coefficients are constant then holomorphy in the open right half plane is established in [EIR] for every continuous representation.

3. The smoothness estimates (11.18) on $T$ can be translated directly into smoothness of $K_{t}$ with respect to the first variable relative to the $\mathcal{L}_{\hat{1}}$-norm. But one can also bound $\left(|g|^{\prime}\right)^{-\gamma}\left\|(I-L(g)) A_{i} T_{t}\right\|_{\hat{2} \rightarrow \infty}$ for $\gamma \in\langle 0,1\rangle$ by a slight variation of the proof of (11.18) and obtain smoothness relative to the $\mathcal{L}_{\hat{2}}$-norm and then by interpolation theory relative to the $\mathcal{L}_{\hat{p}}$-norm with $p \in[2, \infty]$. 


\section{Lower bounds}

We again adopt the assumptions of Sections 10 and 11 and consider lower bounds on the semigroup kernel $\mathrm{K}$. Following the by now standard procedure we first derive lower bounds on $K_{t}(g ; g)$ which are uniform in $g$ and exhibit the anticipated singularity $t^{-D^{\prime} / 2}$ in $t$. Secondly, we convert these bounds into Gaussian bounds by exploiting the convolution semigroup property.

Proposition-12.1 Let $H$ be a subelliptic operator in the form

$$
H=-\sum_{i, j=1}^{d^{\prime}} A_{i} c_{i j} A_{i}+\sum_{i=1}^{d^{\prime}} c_{i} A_{i}+c_{0}
$$

with coefficients $c_{i j}, c_{i} \in L_{\infty ; 1}^{\prime}$ and $c_{0} \in L_{\infty}$ and let $K$ denote the corresponding semigroup kernel.

Then there are $a, \tau>0$ such that

$$
K_{t}(g ; g) \geq a t^{D^{\prime} / 2}
$$

for all $\in\langle 0, \tau]$ where the values of a and $\tau$ depend on the coefficients of $H$ only through $\lambda_{c}$ and the norms $\left\|c_{i j}\right\|_{\infty ; 1}^{\prime},\left\|c_{i}\right\|_{\infty ; 1}^{\prime},\left\|c_{0}\right\|_{\infty}$.

Proof First we consider the operator $H_{0}$ obtained by setting $c_{i}=0$. Let $S^{0}$ and $K^{0}$ denote the corresponding semigroup and kernel. We begin by bounding $K^{0}$ from below. The method we use is a variant of an argument of Varopoulos [Var].

The semigroup $S^{0}$ is symmetric on $L_{2}$ and $\Delta^{1 / 2} S^{0} \Delta^{-1 / 2}$ is symmetric on $L_{\hat{2}}$. If $\widetilde{K}$ is the kernel of $\widetilde{S}$ then

$$
\widetilde{K}_{t}(g ; h)=\Delta(g)^{1 / 2} K_{t}^{0}(g ; h) \Delta(h)^{-1 / 2}
$$

and the symmetry of $\widetilde{S}$ gives

$$
\int_{G} d \hat{g} \int_{G} d \hat{h} \overline{\varphi(g)} \widetilde{K}_{t}(g ; h) \varphi(h)=\left\langle\varphi, \widetilde{S}_{t} \varphi\right\rangle=\left\|\widetilde{S}_{t / 2} \varphi\right\|_{\hat{2}}^{2} \geq 0
$$

Therefore $\widetilde{K}_{t}$ is positive-definite. In particular

$$
\lambda^{2} \widetilde{K}_{t}(g ; g)-2 \lambda \widetilde{K}_{t}(g ; h)+\widetilde{K}_{t}(h ; h) \geq 0
$$


for all $g, h \in G$ and $\lambda \in \mathbf{R}$. Now we average this inequality over the ball $B_{\gamma \sqrt{t}}^{\prime}$ to obtain

$$
\lambda^{2} \widetilde{K}_{t}(g ; g)-2 \lambda\left|B_{\gamma \sqrt{t}}^{\prime}\right|^{-1} \int_{B_{\gamma \sqrt{t}}^{\prime}} d \hat{h} \widetilde{K}_{t}(g ; h)+\left|B_{\gamma \sqrt{t}}^{\prime}\right|^{-1} \int_{B_{\gamma \sqrt{t}}^{\prime}} d \hat{h} \widetilde{K}_{t}(h ; h) \geq 0 .
$$

But the upper bounds of Section 11 give

$$
\widetilde{K}_{t}(h ; h) \leq a t^{-D^{\prime} / 2} e^{\omega t}
$$

and hence

$$
\left|B_{\gamma \sqrt{t}}^{\prime}\right|^{-1} \int_{B_{\gamma \sqrt{t}}^{\prime}} d \hat{h} \widetilde{K}_{t}(h ; h) \leq a e^{\omega} t^{-D^{\prime} / 2}
$$

for all $t \in\{0,1]$. On the other hand

$$
\int_{B_{\gamma \sqrt{t}}^{\prime}} d \hat{h} \widetilde{K}_{t}(g ; h)=\int_{G} d \hat{h} \hat{K}_{t}(g ; h)-\int_{G \backslash B_{\gamma \sqrt{t}}^{\prime}} d \hat{h} \hat{K}_{t}(g ; h)
$$

and by [BrR2] Theorem 2.1.5 one has bounds

$$
\int_{G} d \hat{h} \widetilde{K}_{t}(g ; h) \geq e^{-t\left\|c_{0}\right\|_{\infty}}
$$

In addition it follows from the upper bounds on $\widetilde{K}$, and the fact that $\left|B_{r}^{\prime}\right|$ grows at most exponentially, that for each $\varepsilon>0$ there is a $\gamma>0$ such that

$$
\int_{G \backslash B_{\gamma \sqrt{t}}^{\prime}} d \hat{h} \widetilde{K}_{t}(g ; h)<\varepsilon
$$

for all $t \in\langle 0,1]$. This is verified by the estimation procedure used in the proof of Lemma 4.1. Hence one has estimates

$$
\left|B_{\gamma \sqrt{t}}^{\prime}\right|^{-1} \int_{B_{\gamma \sqrt{t}}^{\prime}} d \hat{h} \widetilde{K}_{t}(g ; h) \geq a^{\prime}\left|B_{\gamma \sqrt{t}}^{\prime}\right|^{-1}
$$

for all $t \in\langle 0,1]$ if $\gamma$ is sufficiently large. But $\left|B_{r}^{\prime}\right|=O\left(r^{D^{\prime}}\right)$ as $r \rightarrow 0$ and hence

$$
\left|B_{\gamma \sqrt{t}}^{\prime}\right|^{-1} \int_{B_{\gamma \sqrt{t}}^{\prime}} d \hat{h} \widetilde{K_{t}}(g ; h) \geq a^{\prime \prime} t^{-D^{\prime} / 2}
$$

for all $t \in\langle 0,1]$ and for a suitable $a^{\prime \prime}>0$. Inserting (12.2) and (12.4) into (12.1) then gives

$$
\lambda^{2} \widetilde{K}_{t}(g ; g)-2 \lambda a^{\prime \prime} t^{-D^{\prime} / 2}+a e^{\omega} t^{-D^{\prime} / 2} \geq 0
$$


for all $\lambda \in \mathbf{R}$. Consequently

$$
\widetilde{K}_{t}(g ; g) \geq\left(\left(a^{\prime \prime}\right)^{2} / a e^{\omega}\right) t^{-D^{\prime} / 2}
$$

for $t \in\langle 0,1]$. Finally as $K_{t}^{0}(g ; g)=\widetilde{K}_{t}(g ; g)$ this gives bounds

$$
K_{t}^{0}(g ; g) \geq a^{\prime} t^{-D^{\prime} / 2}
$$

for $t \in\langle 0,1]$. The value of the positive parameter $a^{\prime}$ depends on the coefficients of $H$ only through the upper bounds on $\widetilde{K}_{t}$ and the norm $\left\|c_{0}\right\|_{\infty}$. Thus $a^{\prime}$ depends only on $\lambda_{c},\left\|c_{i j}\right\|_{\infty ; 1}^{\prime}$, and $\left\|c_{0}\right\|_{\infty}$.

Next we deal with the addition of the first order terms $H_{1}=\sum_{i=1}^{d^{\prime}} c_{i} A_{i}$ by the use of perturbation theory. Let $S$ denote the semigroup corresponding to the operator with the first order terms and $K$ the semigroup kernel. Then the aim is to derive bounds similar to (12.5) for $K$ by showing that the difference between $K_{t}^{0}(g ; g)$ and $K_{t}(g ; g)$ is insignificant compared to $a^{\prime} t^{-D^{\prime} / 2}$. The natural approach via perturbation theory is to demonstrate that the difference between $K_{t}^{0}$ and $K_{t}$ is relatively small with respect to the $\mathcal{L}_{\infty}$-norm. This, however, is equivalent to showing that the cross norm of $S_{t}^{0}-S_{t}$ as an operator from $L_{\hat{1}}$ to $L_{\infty}$ is small. In principle this could be estimated by the methods of the Appendix but this would require bounds on $H_{1} S_{t}^{0}$ as an operator on $L_{\infty}$, as an operator from $L_{\hat{1}}$ to $L_{\infty}$ and as an operator on $L_{\hat{1}}$. One can derive appropriate bounds involving the $L_{\infty}$-space by use of the a priori estimates of Proposition 3.2 but estimates on $L_{\hat{1}}$ would require analogous a priori bounds on $L_{\hat{1}}$. Since we do not have such bounds we adopt an indirect approach via $L_{2}$ estimates.

It follows from Corollary 11.4, the second remark before Proposition 11.3 and the identification

$$
\left\|S_{t}^{0}\right\|_{\hat{q} \rightarrow \infty}=\left\||| K_{t}^{0}\right\| \|_{\hat{p}}
$$

with $1 / p+1 / q=1$ that one has estimates

$$
\begin{array}{rlrl}
\left\|H_{1} S_{t}^{0}\right\|_{\infty \rightarrow \infty} & \leq a t^{-(1+\delta) / 2} e^{\omega t}, & \left\|H_{1} S_{t}^{0}\right\|_{\hat{2} \rightarrow \hat{2}} & \leq a t^{-1 / 2} e^{\omega t}, \\
\left\|H_{1} S_{t}^{0}\right\|_{\hat{2} \rightarrow \infty} & \leq a t^{-D^{\prime} / 4-(1+\delta) / 2} e^{\omega t}, \quad\left\|S_{t}^{0}\right\|_{\infty \rightarrow \infty} \leq a e^{\omega t}, \\
\left\|S_{t}^{0}\right\|_{\hat{2} \rightarrow \hat{2}} & \leq a e^{\omega t}, \\
\left\|S_{t}^{0}\right\|_{\hat{2} \rightarrow \infty} & \leq a t^{-D^{\prime} / 4} e^{\omega t},
\end{array}
$$

where the values of $a$ and $\omega$ depend on $\delta$ and on the coefficients of $H$ through $\lambda_{c}$ and the norms $\left\|c_{i j}\right\|_{\infty ; 1}^{\prime},\left\|c_{i}\right\|_{\infty}$ and $\left\|c_{0}\right\|_{\infty}$. But the kernel $K$ is determined by the kernel $K_{0}$ and the perturbation series expansion

$$
K_{t}=\sum_{n \geq 0} K_{t}^{(n)}
$$


with $K_{t}^{(0)} \equiv K_{t}^{0}$ and

$$
K_{t}^{(n)}=-\int_{0}^{t} d s K_{t-s}^{(0)} * H_{1} K_{s}^{(n-1)}
$$

for $n \geq 1$ where the convolution product is defined by (1.34). Therefore it follows from the above estimates and those of the Appendix that

$$
\left\|K_{t}^{0}-K_{t}\right\|_{\hat{2}} \leq a t^{-D^{\prime} / 4} t^{(1-\delta) / 2} e^{\omega t} .
$$

Now by the assumptions on $H$ the formal adjoint $H^{\dagger}$ has the same general form as $H$ and hence by (1.16) and Proposition 11.6 one has

$$
\begin{aligned}
\left|K_{t}(g ; h)-K_{t}(g ; g)\right| & \leq\left|h g^{-1}\right|^{\prime} k \sup _{1 \leq i \leq d^{\prime}}|| \partial_{i} K_{t} \|_{\infty} \\
& \leq\left|h g^{-1}\right|^{\prime} a t^{-D^{\prime} / 2-(1+\delta) / 2} e^{\omega t}
\end{aligned}
$$

and similarly for $K_{t}^{0}$ where the values of $a$ and $\omega$ depend on $\delta$ and on the coefficients of $H$ through $\lambda_{c}$ and the norms $\left\|c_{i j}\right\|_{\infty ; 1}^{\prime},\left\|c_{i}\right\|_{\infty ; 1}^{\prime},\left\|c_{0}\right\|_{\infty}$. Hence

$$
\left|K_{t}(g ; g)-K_{t}^{0}(g ; g)\right|^{2} \leq 2\left|K_{t}(g ; h)-K_{t}^{0}(g ; g)\right|^{2}+2\left(\left|h g^{-1}\right|^{\prime}\right)^{2} a^{2} t^{-D^{\prime}-(1+\delta)} e^{2 \omega t} \text {. }
$$

Now integrate both sides of this inequality with respect to right invariant Haar measure over $h$ in a ball $B_{g}$ centred at $g$ with subelliptic radius $t^{\alpha}$ where we assume that $\delta$ is sufficiently small that we can choose $(1+\delta) / 2<\alpha<1 / 2+(1-\delta) / D^{\prime}$. Then

$\left|B_{g}\right| \cdot\left|K_{t}(g ; g)-K_{t}^{0}(\dot{g} ; g)\right|^{2} \leq 2||\left|K_{t}-K_{t}^{0}\right| \|\left.\right|_{\hat{2}} ^{2}+2 a^{2} t^{-D^{\prime}-(1+\delta)} e^{2 \omega t} \int_{B_{g}} d \hat{h}\left(\left|h g^{-1}\right|^{\prime}\right)^{2}$ and consequently

$$
\begin{aligned}
& K_{t}(g ; g) \geq K_{t}^{0}(g ; g)- \\
& \quad a\left(\left|B_{g}\right|^{-1 / 2}||\left|K^{t}-K_{t}^{0}\right| \|_{\hat{2}}+t^{-D^{\prime} / 2-(1+\delta) / 2} e^{\omega t}\left(\left|B_{e}\right|^{-1} \int_{B_{e}} d \hat{h}\left(|h|^{\prime}\right)^{2}\right)^{1 / 2}\right)
\end{aligned}
$$

where the redefined $a$ depends on $\delta, \lambda_{c}$ and the norms $\left\|c_{i j}\right\|_{\infty ; 1}^{\prime},\left\|c_{i}\right\|_{\infty ; 1}^{\prime},\left\|c_{0}\right\|_{\infty}$. Since the radius of $B_{e}$ is $t^{\alpha}$ one then has

$$
\left|B_{g}\right|^{1 / 2}=\left|B_{e}\right|^{1 / 2}=\mathrm{O}\left(\left(t^{\alpha}\right)^{D^{\prime} / 2}\right)
$$

and

$$
\left|B_{e}\right|^{-1} \int_{B_{e}} d \hat{h}\left(|h|^{\prime}\right)^{2}=\mathrm{O}\left(t^{2 \alpha}\right)
$$


Thus the lower bound for $K_{t}$ becomes

$$
K_{t}(g ; g) \geq K_{t}^{0}(g ; g)-a t^{-D^{\prime} / 2}\left(t^{(1-\delta) / 2-(\alpha-1 / 2) D^{\prime} / 2}+t^{-(1+\delta) / 2+\alpha}\right) e^{\omega t}
$$

with yet another redefinition of $a$. But by the above choice of $\alpha$ the exponents of $t$ in the middle term on the right are positive and hence one obtains a lower bound

$$
K_{t}(g ; g) \geq a^{\prime} t^{-D^{\prime} / 2}
$$

with $a^{\prime}>0$, for all $t$ in an interval $\langle 0, \tau]$ where the values of both $a^{\prime}$ and $\tau$ depend on the coefficients of $H$ only through $\lambda_{c}$ and the norms $\left\|c_{i j}\right\|_{\infty ; 1}^{\prime},\left\|c_{i}\right\|_{\infty ; 1}^{\prime},\left\|c_{0}\right\|_{\infty}$.

The bounds of Proposition 12.1 have a straightforward extension to $K_{t}(g ; h)$ if $g$ and $h$ are sufficiently close. Using (1.16) one has

$$
\left|K_{t}(h ; g)-K_{t}(g ; g)\right| \leq k\left|h g^{-1}\right|^{\prime} \sup _{1 \leq i \leq d^{\prime}}||\left|A_{i} K_{t}\right| \|_{\infty}
$$

and hence by Proposition 11.6 and (12.5)

$$
K_{t}(h ; g) \geq\left(a^{\prime}-a_{\delta}^{\prime \prime}\left|h g^{-1}\right|^{\prime} t^{-(1+\delta) / 2}\right) t^{-D^{\prime} / 2}
$$

for all $t \in\langle 0, \tau], \delta>0$ and a suitable $a_{\delta}^{\prime \prime}>0$. Consequently

$$
K_{t}(g ; h) \geq\left(a^{\prime} / 2\right) t^{-D^{\prime} / 2}
$$

for all $t \in\langle 0, \tau]$ and $g, h \in G$ with $\left|g h^{-1}\right|^{\prime} \leq r t^{(1+\delta) / 2}$ where $r=a^{\prime} / 2 a_{\delta}^{\prime \prime}$.

The estimate (12.6) is the key to obtaining Gaussian lower bounds on the semigroup kernels by a now standard argument based on the convolution semigroup property. The method gives proper Gaussian bounds, however, only if $\delta=0$. But the parameter $\delta$ enters through the use of the a priori bounds of Proposition 3.2 to estimate the derivatives $A_{i} K_{t}$. If the leading coefficients $c_{i j} \in L_{\infty ; 3}^{\prime}$ then these bounds are valid with $\delta=0$ by Proposition 6.4. Thus under this additional smoothness condition (12.6) holds for $\left|g h^{-1}\right|^{\prime} \leq r t^{1 / 2}$ and $t \in\langle 0, \tau]$ and one can derive Gaussian bounds.

Proposition 12.2 Let $H$ be a subelliptic operator in the form

$$
H=-\sum_{i, j=1}^{d^{\prime}} A_{i} c_{i j} A_{i}+\sum_{i=1}^{d^{\prime}} c_{i} A_{i}+c_{0}
$$

with coefficients $c_{i j} \in L_{\infty ; 3}^{\prime}, c_{i} \in L_{\infty ; 1}^{\prime}$ and $c_{0} \in L_{\infty}$ and let $K$ denote the corresponding semigroup kernel. 
Then there exist $a, b^{\prime}>0$, and $\omega^{\prime} \geq 0$, such that

$$
K_{t}(g ; h) \geq a t^{-D^{\prime} / 2} e^{-\omega^{\prime} t} e^{-b^{\prime}\left(\left|g h^{-1}\right|^{\prime}\right)^{2} t^{-1}}
$$

for all $g, h \in G_{0}$ and $t \geq 0$. The values of $a, b^{\prime}$, and $\omega^{\prime}$ depend on the coefficients of $H$ through the parameters $\lambda_{c},\left\|c_{i j}\right\|_{\infty ; 3}^{\prime},\left\|c_{i}\right\|_{\infty ; 1}^{\prime}$ and $\left\|c_{0}\right\|_{\infty}$.

Proof Fix $g, h \in G$, and $t \geq 0$. Following [JeS] one deduces by an easy compactness argument that for each $n$ one may choose a sequence $g_{0}=g, g_{1}, \ldots, g_{n}=h$ such that $\left|g_{i} g_{i+1}^{-1}\right|^{\prime}=\left|g h^{-1}\right|^{\prime} / n$. Then

$$
K_{t}(g ; h) \geq \int_{V_{1}} d \hat{h}_{1} \ldots \int_{V_{n-1}} d \hat{h}_{n-1} K_{t / n}\left(g ; h_{1}\right) \ldots K_{t / n}\left(h_{n-1} ; h\right)
$$

where $V_{i}$ is a subelliptic ball of radius $r_{n}$ around $g_{i}$, i.e.,

$$
V_{i}=\left\{h \in G ;\left|g_{i} h^{-1}\right|^{\prime} \leq r_{n}\right\}
$$

Now for the best possible estimate we make $r_{n}$ as large as possible compatible with (12.6), i.e., recalling that one now has $\delta=0$ one requires that in the integrand only $h_{i}$ with $\left|h_{i} h_{i+1}^{-1}\right|^{\prime} \leq r(t / n)^{1 / 2}$ occur. But using

$$
\left|h_{i} h_{i+1}^{-1}\right|^{\prime} \leq\left|h_{i} g_{i}^{-1}\right|^{\prime}+\left|g_{i} g_{i+1}^{-1}\right|^{\prime}+\left|g_{i+1} h_{i+1}^{-1}\right|^{\prime}
$$

this leads to the condition

$$
2 r_{n}+\left|g h^{-1}\right|^{\prime} / n=r(t / n)^{1 / 2}
$$

for the optimal value of $r_{n}$. Hence if we choose $n$ large enough that

$$
\left|g h^{-1}\right|^{\prime} / n=r(t / n)^{1 / 2} / 2
$$

then

$$
r_{n} \geq r(t / n)^{1 / 2} / 4
$$

But to use (12.6) we must also choose $n$ large enough that $t / n \leq \tau$ and the two conditions on $n$ are then $n \geq t / \tau$ and

$$
n \geq\left(2\left|g h^{-1}\right|^{\prime} / r t^{1 / 2}\right)^{2}=b^{\prime \prime}\left(\left|g h^{-1}\right|^{\prime}\right)^{2} t^{-1}
$$

Now (12.6) and the convolution estimate on the kernel give

$$
K_{t}(g ; h) \geq\left(\left(a^{\prime} / 2\right) n^{D^{\prime} / 2} t^{-D^{\prime} / 2}\right)^{n} V\left(r_{n}\right)^{n-1}
$$


where $V(\lambda)$ now denotes the Haar measure of the ball $\left\{g \in G ;|g|^{\prime} \leq \lambda\right\}$. Since $V(\lambda) \geq b \lambda^{D^{\prime}}$ for some $b \geq 0$ and all small $\lambda$ this gives

$$
\begin{aligned}
K_{t}(g ; h) & \geq\left(\left(a^{\prime} / 2\right)(t / n)^{-D^{\prime} / 2}\right)^{n}\left(b\left(r(t / n)^{1 / 2} / 4\right)^{D^{\prime}}\right)^{n-1} \\
& =\left(a^{\prime} / 2\right) t^{-D^{\prime} / 2} n^{D^{\prime} / 2}\left(\left(a^{\prime} b / 2\right)(r / 4)^{D^{\prime}}\right)^{n-1} .
\end{aligned}
$$

But the last term is the $(n-1)$-th power of a small value and hence for the best estimate we choose $n$ to have the smallest value compatible with the above conditions, i.e.,

$$
n-1 \leq(t / \tau) \vee b^{\prime \prime}\left(\left|g h^{-1}\right|^{\prime}\right)^{2} t^{-1} \leq n .
$$

Hence one has a lower bound of the form

$$
\begin{aligned}
\bar{K}_{t}(g ; h) & \geq\left(a^{\prime} / 2\right) t^{-D^{\prime} / 2}\left(\left(a^{\prime} b / 2\right)(r / 4)^{D^{\prime}}\right)^{\left(t / \tau+b^{\prime \prime}\left(\left|g h^{-1}\right|^{\prime}\right)^{2} t^{-1}\right)} \\
& \geq a t^{-D^{\prime} / 2} \exp \left\{-b^{\prime}\left(b^{\prime \prime}\left(\left|g h^{-1}\right|^{\prime}\right)^{2} t^{-1}+t / \tau\right)\right\} \\
& \geq a t^{-D^{\prime} / 2} e^{-b\left(\left|g h^{-1}\right|^{\prime}\right)^{2} t^{-1}} e^{-\omega t}
\end{aligned}
$$

for suitable constants $a, b>0$ and $\omega \geq 0$.

The parameters $a, b$, and $\omega$, in the bounds only depend on the coefficients through $\lambda_{c},\left\|c_{i j}\right\|_{\infty ; 3}^{\prime},\left\|c_{i}\right\|_{\infty ; 1}^{\prime}$ and $\left\|c_{0}\right\|_{\infty}$, because the proof was based on Proposition 6.4 and the upper bounds on the kernel.

Remark If one argues in the identical fashion but with the $\delta>0$ version of (12.6) then one finds bounds

$$
K_{t}(g ; h) \geq\left(a^{\prime} / 2\right) t^{-D^{\prime} / 2} n^{D^{\prime} / 2}\left(\left(a^{\prime} b / 2\right)(r / 4)^{D^{\prime}}\right)^{n-1}(t / n)^{\delta(n-1) D^{\prime} / 2}
$$

with the optimal value of $n$ determined by

$$
n-1 \leq(t / \tau) \vee b^{\prime \prime}\left(\left|g h^{-1}\right|^{\prime}\right)^{2 /(1-\delta)} / t^{(1+\delta) /(1-\delta)} \leq n .
$$

These bounds are valid under the weaker assumption that $c_{i j} \in L_{\infty ; 1}^{\prime}$. The extra $\delta$-dependent contribution, however, makes the bounds more complicated than the Gaussian bounds derived when $\delta=0$. 


\section{Conclusion}

The result stated in the introduction, Theorem 1.1, has now been established and much more information has been gleaned from the foregoing analysis. Under the hypotheses of Statement 1 of the theorem the subelliptic operator $H$ can be written in the partially asymmetric form covered by Propositions 11.7 and 11.8. Then the statement is a direct consequence of these propositions. Proposition 11.2 also gives additional information on the regularity of the action of the semigroup and Corollary 11.4 and Proposition 11.7 describe smoothness properties of the associated kernel $K$. Statement 2 of Theorem 1.1 follows from Proposition 6.2. Moreover, Proposition 8.2 gives conditions on the coefficients of $H$ which ensure that the generator of the semigroup restricted to $L_{p}$ is the $L_{p}$-closure of $H$. Note that this proposition places rather stringent conditions on the first-order coefficients but these are largely spurious. In fact on the $L_{p}$-spaces with $p \in[2, \infty]$ no smoothness of the first-order coefficients is required. This follows from the a priori estimates of Proposition 3.1 and 3.2 together with perturbation theory and an interpolation argument. It is probable that the same is true for $p \in[1,2]$ and this would follow if one had suitable a priori inequalities on the $L_{1^{-}}$, and $L_{1^{-}}$, spaces. In particular this would follow if one could verify the following analogue of the $L_{\infty}$-conjecture stated in the introduction.

$L_{\hat{1}}$-conjecture If $H$ is a subelliptic operator of the form (1.17) with $c_{\alpha} \in L_{\infty ; 1}^{\prime}$ whenever $|\alpha|=2$ then there is an $a>0$, whose value depends on the coefficients of $H$ only through the parameters $\lambda_{c}$ and $\|c\|_{1}^{\prime}$, such that

$$
N_{\hat{1} ; 1}^{\prime}(\varphi) \leq \varepsilon\|H \varphi\|_{\hat{1}}+a \varepsilon^{-1}\|\varphi\|_{\hat{1}}
$$

for all $\varphi \in L_{\hat{1} ; 2}^{\prime}$

Although we have formulated this conjecture on $L_{\hat{1}}$ it is equivalent to the analogous statement on $L_{1}$ by the argument used to prove the equivalence of (3.5) and (3.6) in the proof of Proposition 3.1. Again the conjecture seems eminently reason- 
able, e.g., it is true in the strongly elliptic case, but experience indicates that it is probably more difficult to establish than the $L_{\infty}$-conjecture.

The lower bounds in Statement 3 of Theorem 1.1 are established in Proposition 12.2. These could also be improved if the $L_{\infty}$ and $L_{\hat{1}}$ conjectures were both valid. Then the bounds would follow from the assumptions $c_{\alpha} \in L_{\infty ; 1}^{\prime}$ whenever $|\alpha|=2$ with no smoothness required for the lower order coefficients. The only change is to the proof of Proposition 12.1. Then the perturbation argument could be used directly to bound the $\mathcal{L}_{\infty}$-norm of the difference $K_{t}-K_{t}^{0}$.

Finally we note that although this article has been restricted to real coefficients a number of the results also hold for complex coefficients. The case of complex constant coefficients is covered in [EIR2] and complex variable coefficients require an amalgamation of the techniques of the two articles. 


\section{A Appendix}

In this appendix we examine a number of coupled integral inequalities which are useful for the estimation of convergence of the time dependent perturbation theory expansion encountered in the main text. We consider a semigroup $S$, a small perturbation $H_{1}$ of its generator and the perturbation series

$$
T_{t}=\sum_{n \geq 0} T_{t}^{(n)}
$$

where

$$
T_{t}^{(0)}=S_{t}
$$

and

$$
T_{t}^{(n)}=\int_{0}^{t} d s T_{t-s}^{(0)} H_{1} T_{s}^{(n-1)}
$$

for $n=0,1,2, \ldots$. The functions $a_{n}, b_{n}$, etc. in the following proposition are typically given by

$$
\begin{aligned}
& a_{n}(t)=\left\|T_{t}^{(n)}\right\|_{\hat{p} \rightarrow \hat{p}}, \quad b_{n}(t)=\left\|H_{1} T_{t}^{(n)}\right\|_{\hat{p} \rightarrow \hat{p}}, \\
& c_{n}(t)=\left\|T_{t}^{(n)}\right\|_{\hat{r} \rightarrow \hat{r}}, \quad d_{n}(t)=\left\|H_{1} T_{t}^{(n)}\right\|_{\hat{r} \rightarrow \hat{r}}, \\
& f_{n}(t)=\left\|T_{t}^{(n)}\right\|_{\hat{r} \rightarrow \hat{p}}, \quad g_{n}(t)=\left\|H_{1} T_{t}^{(n)}\right\|_{\hat{r} \rightarrow \hat{p}},
\end{aligned}
$$

where $1 \leq r<p \leq \infty$

Proposition A.1 Let $a_{n}, b_{n}, \ldots, g_{n}, n=0,1,2, \ldots$, denote non-negative functions on $\mathbf{R}_{+}$and assume they satisfy the following system of integral inequalities:

$$
\begin{aligned}
& a_{n}(t) \leq \int_{0}^{t} d s a_{0}(t-s) b_{n-1}(s) \\
& b_{n}(t) \leq \int_{0}^{t} d s b_{0}(t-s) b_{n-1}(s) \\
& c_{n}(t) \leq \int_{0}^{t} d s c_{0}(t-s) d_{n-1}(s) \\
& d_{n}(t) \leq \int_{0}^{t} d s d_{0}(t-s) d_{n-1}(s)
\end{aligned}
$$




$$
\begin{aligned}
& f_{n}(\bar{t}) \leq \int_{0}^{t} d s\left\{\left(f_{0}(t-s) d_{n-1}(s)\right) \wedge\left(a_{0}(t-s) g_{n-1}(s)\right)\right\} \\
& g_{n}(t) \leq \int_{0}^{t} d s\left\{\left(g_{0}(t-s) d_{n-1}(s)\right) \wedge\left(b_{0}(t-s) g_{n-1}(s)\right)\right\}
\end{aligned}
$$

for $=1,2, \ldots$ and all $t>0$. Assume also that one has bounds

$$
\begin{aligned}
& a_{0}(t) \leq a e^{\omega t} t^{m_{1}}, \quad b_{0}(t) \leq a e^{\omega t} t^{m_{2}}, \\
& c_{0}(t) \leq a e^{\omega t} t^{m_{3}}, d_{0}(t) \leq a e^{\omega t} t^{m_{4}}, \\
& f_{0}(t) \leq a e^{\omega t} t^{m_{5}}, g_{0}(t) \leq a e^{\omega t} t^{m_{6}},
\end{aligned}
$$

for all $t>0$, where $m_{1}, \ldots, m_{6}$ are real numbers and $m_{i}>-1$ for $i=1,2,3,4$.

It follows that the series

$$
\begin{aligned}
a(t) & =\sum_{n \geq 0} a_{n}(t), \quad b(t)=\sum_{n \geq 0} b_{n}(t), \\
c(t) & =\sum_{n \geq 0} c_{n}(t), \quad d(t)=\sum_{n \geq 0} d_{n}(t), \\
f(t) & =\sum_{n \geq 0} f_{n}(t), \quad g(t)=\sum_{n \geq 0} g_{n}(t),
\end{aligned}
$$

converge for all $t>0$, uniformly on compact intervals of $\mathbf{R}_{+}$, and their sums satisfy estimates

$$
\begin{aligned}
& a(t) \leq A t^{m_{1}} e^{\omega^{\prime} t}, \quad b(t) \leq A t^{m_{2}} e^{\omega^{\prime} t}, \\
& c(t) \leq A t^{m_{3}} e^{\omega^{\prime} t}, d(t) \leq A t^{m_{4}} e^{\omega^{\prime} t}, \\
& f(t) \leq A t^{m_{5}} e^{\omega^{\prime} t}, g(t) \leq A t^{m_{6}} e^{\omega^{\prime} t},
\end{aligned}
$$

for all $t>0$ where $A$ and $\omega^{\prime}$ are constants whose values depend on $a, \omega$ and the $m_{i}$.

Proof First recall that the factorial function is defined on $\langle-1, \infty\rangle$ by the

$$
n !=\int_{0}^{\infty} d u e^{-u} u^{n}
$$

and that the relation

$$
\int_{0}^{t} d s(t-s)^{m} s^{n}=\frac{m ! n !}{(m+n+1) !} t^{m+n+1}
$$

is valid for all $m, n>-1$.

Next we postulate that the $b_{n}(t)$ satisfy bounds

$$
b_{n}(t) \leq b_{n} e^{\omega t} t^{m_{2}+n\left(1+m_{2}\right)}
$$

for all $t>0$ and suitable $b_{n}>0$. Then it follows from the integral inequalities that the $b_{n}(t)$ indeed satisfy the bounds provided that

$$
b_{n} \leq b_{0} b_{n-1} \frac{m_{2} !\left(m_{2}+(n-1)\left(1+m_{2}\right)\right) !}{\left(m_{2}+n\left(1+m_{2}\right)\right) !} .
$$


Therefore-

$$
b_{n}(t) \leq a^{n+1}\left(m_{2} !\right)^{n+1} e^{\omega t} t^{m_{2}+n\left(1+m_{2}\right)} /\left(m_{2}+n\left(1+m_{2}\right)\right) !
$$

and then by redefinition of $a$ and a suitable choice of $b$ one has bounds

$$
b_{n}(t) \leq a t^{m_{2}}\left(b^{n} t^{n} / n !\right)^{1+m_{2}} e^{\omega t} .
$$

Consequently

$$
b(t) \leq a t^{m_{2}} e^{\omega t} \sum_{n \geq 0}\left(b^{n} t^{n} / n !\right)^{1+m_{2}} .
$$

But if $1+m_{2}<1$ the one may use Hölder's inequality to bound the sum by a suitable multiple of an exponential. Alternatively if $1+m_{2}>1$ one may split the sum into two parts consisting of terms for which $b^{n} t^{n} / n !>1$ and the remaining terms with $b^{n} t^{n} / n ! \leq 1$. The first sum, however, is bounded by $\exp \left(\left(1+m_{2}\right) b t\right)$ and the second by $\exp (b t)$ and so one has the desired bounds

$$
b(t) \leq a t^{m_{2}} e^{\omega^{\prime} t} .
$$

It is also evident from these estimates that the series converges uniformly on compact intervals of the positive real line.

Next using (A.1) and (A.2) together with the integral inequality for the $a_{n}$ and the bound on $a_{0}$ one finds

$$
\begin{aligned}
a_{n}(t) & \leq \frac{a^{n+1}\left(m_{2} !\right)^{n}}{\left(m_{2}+n\left(1+m_{2}\right)\right) !} e^{\omega t} \int_{0}^{t} d s(t-s)^{m_{1}} s^{m_{2}+(n-1)\left(1+m_{2}\right)} \\
& =\frac{a^{n+1}\left(m_{2} !\right)^{n} m_{1} !}{\left(m_{1}+n\left(1+m_{2}\right)\right) !} e^{\omega t} t^{m_{1}+n\left(1+m_{2}\right)}
\end{aligned}
$$

and again one concludes that one has bounds

$$
a_{n}(t) \leq a t^{m_{1}}\left(b^{n} t^{n} / n !\right)^{1+m_{2}} e^{\omega t} .
$$

and consequently

$$
a(t) \leq a t^{m_{2}} e^{\omega^{\prime} t} .
$$

Now replacing $a_{n}$ and $b_{n}$ by $c_{n}$ and $d_{n}$ in the above arguments one deduces the desired bounds on $c$ and $d$. Moreover, in the course of the proof one obtains the estimates

$$
\begin{aligned}
& d_{n}(t) \leq a^{n+1}\left(m_{4} !\right)^{n+1} e^{\omega t} t^{m_{4}+n\left(1+m_{4}\right)} /\left(m_{4}+n\left(1+m_{4}\right)\right) ! \\
& c_{n}(t) \leq a^{n+1}\left(m_{4} !\right)^{n} m_{3} ! e^{\omega t} t^{m_{3}+n\left(1+m_{4}\right)} /\left(m_{3}+n\left(1+m_{4}\right)\right) !
\end{aligned}
$$


which will-be instrumental in the proof of the bounds on $f_{n}$ and $g_{n}$.

Next we postulate that one has bounds

$$
g_{n}(t) \leq g_{n} t^{\tilde{t}_{n}} e^{\omega t}
$$

with $\tilde{g}_{0}=m_{6}$. It follows from the integral inequality for the $g_{n}$ that

$$
g_{n}(t) \leq \int_{0}^{t / 2} d s g_{0}(t-s) d_{n-1}(s)+\int_{t / 2}^{t} d s b_{0}(t-s) g_{n-1}(s) .
$$

Using the bounds for $b_{0}$ and $d_{n-1}$ one then deduces that the $g_{n}(t)$ indeed satisfy the bounds with

$$
\tilde{g}_{n}=\left\{\left(m_{6}+m_{4}+(n-1)\left(1+m_{4}\right)\right) \wedge\left(m_{2}+\tilde{g}_{n-1}\right)\right\}+1 .
$$

Repetition of this argument establishes that the degree $\tilde{g}_{n}$ of $g_{n}$ increases by $\left(1+m_{4}\right) \wedge$ $\left(1+m_{2}\right)$ at each step. Hence after a finite number of steps $\tilde{g}_{n-1}>-1$. Thereafter one may use the integral inequality

$$
g_{n}(t) \leq \int_{0}^{t} d s b_{0}(t-s) g_{n-1}(s)
$$

and deduce the required bounds on $g_{n}(t)$ by repetition of the previous reasoning. Furthermore one can show that

$$
g(t)=\sum_{n=0}^{\infty} g_{n}(t) \leq A t^{m_{6}} e^{\omega^{\prime} t}
$$

by essentially repeating the argument for $b(t)$.

Finally one bounds $f_{n}(t)$ by assuming that it satisfies an ansatz similar to that used for $g_{n}(t)$ and then uses

$$
f_{n}(t) \leq \int_{0}^{t / 2} d s f_{0}(t-s) d_{n-1}(s)+\int_{t / 2}^{t} d s a_{0}(t-s) g_{n-1}(s) .
$$

for the initial iterative steps, until $\tilde{g}_{n-1}>-1$. Subsequently, one uses the second set of inequalities

$$
f_{n}(t) \leq \int_{0}^{t} d s a_{0}(t-s) g_{n-1}(s)
$$

and proceeds as before. 


\section{References}

[ABR] Arendt, W., Batty, C. J. K. and D. W. Robinson; Positive semigroups generated by elliptic operator on Lie groups. J. Op. Th. 23 (1990) 369-407

[Bon] Bony, J. M., Principe de maximum, inégalité de Harnack et unicité du problème de Cauchy pour les opérateurs elliptiques dégénérés, Ann. Inst. Fourier (Grenoble) 19 (1969) 277-304

[BrR1] Bratteli, O. and D. W. Robinson; Second-order elliptic operators and heat kernels on Lie groups. Trans. Amer. Math. Soc. 325 (1991) 683-713

[BrR2] Bratteli, O. and D. W. Robinson; Operator algebras and quantum statistical mechanics I (Second edition) Springer Verlag (1987)

[BuB] Butzer, P.L. and H. Berens; Semi-groups of operators and approximation. Springer-Verlag (1967)

[Car] Carathéodory, C; Untersuchungen Über die Grundlagen der Thermodynamik. Math. Ann. 67 (1909) 355-386

[CFKS] Cycon, H. L., Froese, R. G., Kirsch, W. and B. Simon; Schrödinger operators. Springer-Verlag (1987)

[CKS] Carlen, E. A., Kusuoka, A., and D. W. Stroock; Upper bounds for symmetric Markov transition functions. Ann. Inst. Henri Poincaré. Prob. et Stat. 2 (1987) $245-287$

[Dav1] Davies, E. B.; Explicit constants for Gaussian upper bounds on heat kernels. Amer. J. Math. 109 (1987) 545-570

[Dav2] Davies, E. B.; Pointwise bounds on the space and time derivatives of heat kernels. J. Op. Th. 21 (1989) 367-378

[DeLM] DeLeeuw, K. and H. Mirkil; A priori estimates for differential operators in $L_{\infty}$-norms. Ill. J. Math. 8 (1964) 112-124. 
[ElR1] ter-Elst, A. F. M. and D. W. Robinson; Subelliptic operators on Lie groups: regularity. Preprint, Australian National University, CMA-MR12-91

[ElR2] ter Elst, A. F. M. and D. W. Robinson; Complex subelliptic operators on Lie groups. Preprint, Australian National University, CMA-MR?-92

[FaS] Fabes, E. B. and D. W. Stroock; A new proof of Moser's Harnack inequality using the old ideas of Nash. Arch. Rat. Mech. Anal. 96 (1986) 327-338

[JeS] Jerison, D. S. and A. Sánchez-Calle; Estimates for the heat kernel for a sum of squares of vector fields. Ind. Univ. J. Math. 35 (1986) 835-854

[Jör] Jörgensen, P. E. T.; Representations of differential operators on a Lie group. J. Funct. Anal. 20 (1975) 105-135

[NoS] Norris, J. R., and D. W. Stroock; Estimates on the fundamental solution to heat flows with uniformly elliptic coefficients. Preprint (1990)

[Orn] Ornstein, D.; A non-inequality for differential operators in the $L_{1}$-norm. Arch. Rat. Mech. Anal. 11 (1962) 40-49.

[Ouh] Ouhabaz, E-M.; $L^{\infty}$-contractivity of semigroups generated by sectorial forms (preprint Besançon 1990)

[Paz] Pazy, A.; Semigroups of linear operators and applications to partial differential equations. Springer-Verlag (1983)

[ReS] Reed, M. and B. Simon; Methods of modern mathematical physics II. Academic Press (1975)

[Rob1] Robinson, D. W.; Elliptic operators and Lie groups. Oxford Univ. Press (1991)

[Rob2] Robinson, D. W.; Elliptic differential operators on Lie Groups. J. Funct. Anal. 97 (1991) 373-402

[RoS] Rothschild, L. P. and E. M. Stein; Hypoelliptic differential operators and nilpotent groups. Acta Math. 137 (1977) 247-320

[SaS] Saloff-Coste, L. and D. W. Stroock; Operateurs uniformement sous-elliptiques sur les groupes de Lie. J. Funct. Anal. 
[San] Sánchez-Calle, A; Fundamental solutions and geometry of the sum of squares of vector fields. Inv. Math. 78 (1984) 143-160

[Ste] Stewart, B.; Generation of analytic semigroups by strongly elliptic operators. Trans. Amer. Math. Soc. 199 (1974) 141-161

[Str] Stroock, D. W.; Estimates on the heat kernel for second order divergence form operators. Lecture notes (1990)

[Trè] Trèves, F.; Introduction to pseudodifferential and Fourier integral operators $I$. Plenum Press (1980)

[Var] Varopoulos, N. Th.; Analysis on Lie groups. J. Funct. Anal. 76 (1988) 346-410

[VSC] Varopoulos, N: Th., Saloff-Coste, L. and T. Coulhon; Analyse sur les groupes de Lie (to be published)

[Yos] Yosida, K.; Functional Analysis. Fourth Edition. Springer Verlag (1974)

Ola Bratteli

Institute of Mathematics

University of Trondheim

N-7034 Trondheim

Norway

Present address:

Department of Mathematics

University of Oslo

P.B. 1053-Blindern

N-0316 Oslo 3

Norway
Derek W. Robinson

Centre for Mathematics and its Applications

School of Mathematical Sciences

Australian National University

GPO Box 4

Canberra, ACT 2601

Australia 